Supporting Information for

\title{
Development of Quinoline-Derived Chiral Diaminocarbene Ligands and Their Transition Metal Complexes: Synthesis, Structural Characterization, and Catalytic Properties
}

Cong Xu, ${ }^{\mathrm{a}}$ Yu Feng, ${ }^{\mathrm{a}}$ Li-Ren Wang, ${ }^{\mathrm{a}}$ Wen-Peng Ma, ${ }^{\mathrm{a}}$ Yan-Mei He, ${ }^{\mathrm{a}}$ and Qing-Hua Fan ${ }^{\mathrm{a}, \mathrm{b}^{*}}$

aBeijing National Laboratory for Molecular Sciences, CAS Key Laboratory of Molecular Recognition and Function, Institute of Chemistry, Chinese Academy of Sciences (ICCAS), and University of Chinese Academy of Sciences, Beijing 100190 (P. R. China)

bCollaborative Innovation Center of Chemical Science and Engineering, Tianjin 300072 (P. R. China)

E-mail: fengyu211@iccas.ac.cn; fanqh@iccas.ac.cn

\section{Contents}

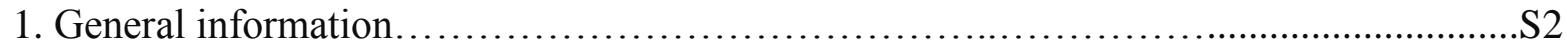

2. Addition Experiments......................................................... 3

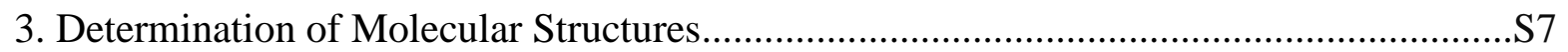

4. General procedure for the synthesis of substrates....................................... 19

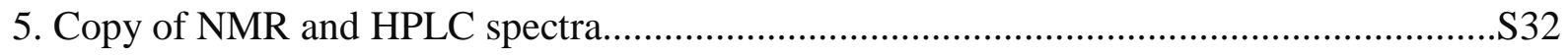

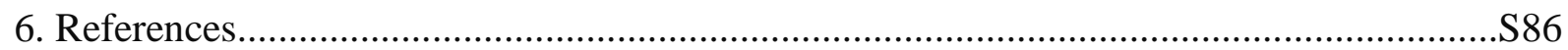




\section{General information}

Unless otherwise noted, all experiments were carried out under an atmosphere of nitrogen using standard Schlenk techniques or in a nitrogen-filled glovebox. ${ }^{1} \mathrm{H}$ NMR and ${ }^{13} \mathrm{C}$ NMR spectra were recorded on Bruker Model Avance DMX 300 Spectrometer $\left({ }^{1} \mathrm{H} 300 \mathrm{MHz}\right.$ and ${ }^{13} \mathrm{C} 75 \mathrm{MHz}$, respectively), Bruker Model Avance DMX 400 Spectrometer $\left({ }^{1} \mathrm{H} 400 \mathrm{MHz}\right.$ and ${ }^{13} \mathrm{C} 100 \mathrm{MHz}$, respectively) and Bruker Model Avance DMX 500 Spectrometer $\left({ }^{1} \mathrm{H} 500\right.$ $\mathrm{MHz}$ and ${ }^{13} \mathrm{C} 125 \mathrm{MHz}$, respectively). Chemical shifts $(\delta)$ were given in ppm and were referenced to residual solvent or TMS peaks. Optical rotations were measured with Rudolph Autopl VI polarimeter. High resolution MS (P-ESI HRMS) were obtained on Thermo Fisher Q Exactive Mass Spectrometer. IR spectrums were recorded on Thermo Fisher Scientific Nicolet FT-6700 spectrometer, wavenumbers $(v)$ were given in $\mathrm{cm}^{-1}$. HPLC analyses were performed on a Varian Prostar 210 liquid chromatography. All organic solvents were dried using standard, published methods and were distilled before use. All other chemicals were used as received from Aldrich or Acros without further purification. The catalysts were

prepared according to the published method. ${ }^{[1]}$ All substrates ${ }^{[2,3,4]}$ were synthesized according to the modified literature methods. 


\section{Additional Experiments}

Scheme S1. Scale-up of the Asymmetric Hydrogenation of 2,2'-Bisquinoline Derivatives. ${ }^{\text {[a] }}$

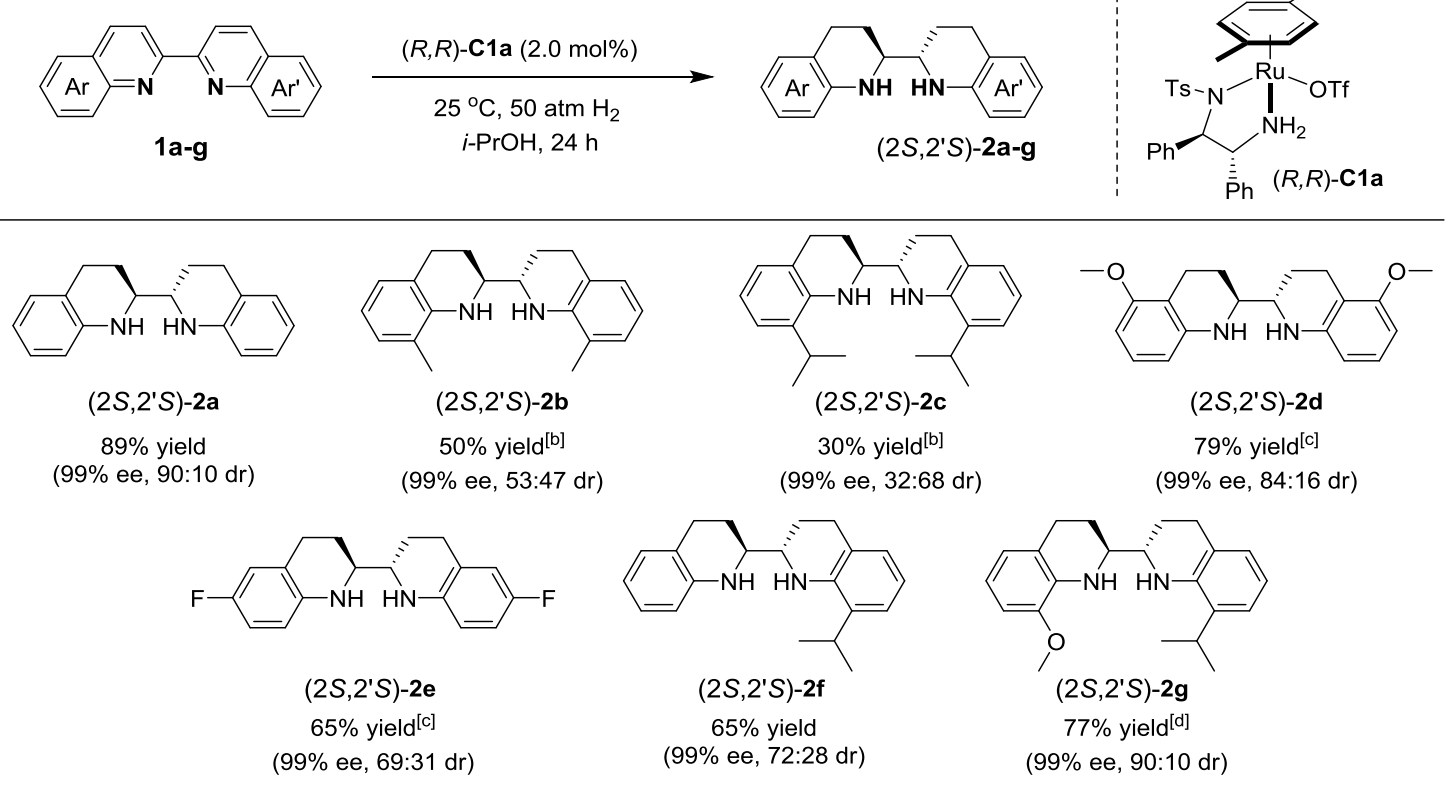

${ }^{\mathrm{a}}$ Reaction conditions: Substrate $(0.78 \mathrm{mmol})$ in $i-\mathrm{PrOH}(10.0 \mathrm{~mL}),(R, R)-\mathbf{C 1 a}(2.0 \mathrm{~mol} \%), \mathrm{H}_{2}(50 \mathrm{~atm})$, stirred at $25^{\circ} \mathrm{C}$ for $24 \mathrm{~h}$. Yields of isolated product are given. The ee values were determined by HPLC with a chiral stationary phase. ${ }^{\mathrm{b}}(R, R)-\mathrm{C1a}(4.0 \mathrm{~mol} \%), 75{ }^{\circ} \mathrm{C} .{ }^{\mathrm{c}}(R, R)-\mathrm{C1a}(4.0 \mathrm{~mol} \%), 50{ }^{\circ} \mathrm{C}, 48 \mathrm{~h}$. ${ }^{\mathrm{d}}(R, R)-\mathbf{C 1 a}(4.0 \mathrm{~mol} \%), 50{ }^{\circ} \mathrm{C}$. 
Scheme S2. Scale-up of the Asymmetric Hydrogenation of 2-(pyridin-2-yl)quinoline Derivatives. ${ }^{\text {[a] }}$

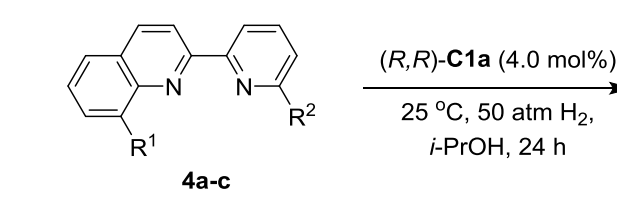

Method II:

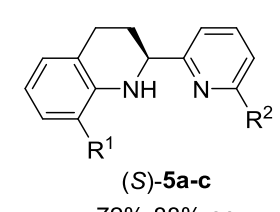

$72 \%-88 \%$ ee

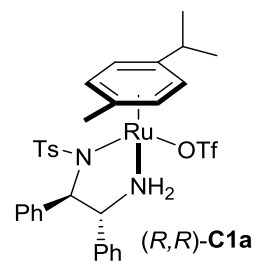

$\overline{\overline{\mathrm{P}}}(R, R)-\mathrm{C} 1 \mathrm{a}$

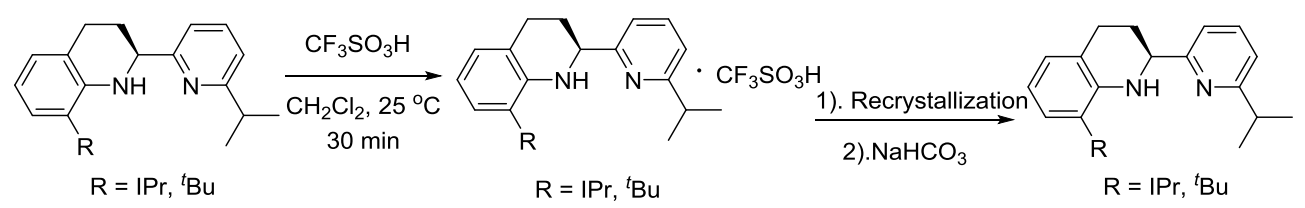

(S)-5b-c

$72 \%-88 \%$ ee

$(\mathrm{S})-\mathbf{5 b}-\mathbf{c} \cdot \mathrm{CF}_{3} \mathrm{SO}_{3} \mathrm{H}$

(S)-5b-c

$99 \%$ ee

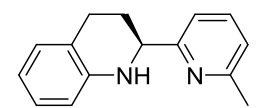

(S)-5a

$99 \%$ yield, $87 \%$ ee

$79 \%$ yield, $99 \%$ ee $^{[b]}$

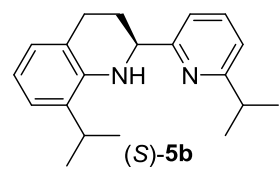

$72 \%$ yield, $88 \%$ ee $47 \%$ yield, $99 \%$ ee $^{[c]}$

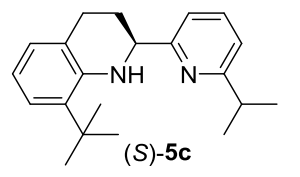

$87 \%$ yield, $72 \%$ ee $23 \%$ yield, $99 \%$ ee $^{[\mathrm{d}]}$

${ }^{\mathrm{a}}$ Reaction conditions: Substrate $(4.0 \mathrm{mmol})$ in $i-\mathrm{PrOH}(10.0 \mathrm{~mL}),(R, R)-\mathrm{C1a}(4.0 \mathrm{~mol} \%), \mathrm{H}_{2}(50 \mathrm{~atm})$, stirred at $25^{\circ} \mathrm{C}$ for $24 \mathrm{~h}$. Yields of isolated product are given. The ee values were determined by HPLC with a chiral stationary phase. ${ }^{\mathrm{b}} 99 \%$ ee was achieved via recrystallization from ethyl acetate/ petroleum ether. ${ }^{\text {c9 } 99 \% ~ e e ~ w a s ~ a c h i e v e d ~ v i a ~ r e c r y s t a l l i z a t i o n ~ f r o m ~ e t h y l ~ a c e t a t e / p e t r o l e u m ~ e t h e r ~}$ according to the method II. ${ }^{\mathrm{d}} 99 \%$ ee was achieved via twice recrystallization from ethyl acetate/petroleum ether according to the method II. 
Table S1. Synthesis of chiral 6a: optimizing reaction conditions. ${ }^{[a]}$

\begin{tabular}{|c|c|c|c|c|c|}
\hline \multicolumn{2}{|c|}{$\begin{array}{l}(S)-5 a \\
99 \% \text { ee }\end{array}$} & $(S, R, R)-\mathbf{6 a}$ & \multicolumn{2}{|c|}{$(S, S, S)-6 \mathbf{a}^{\prime}$} & \multirow{2}{*}{$\begin{array}{c}\text { 6a" } \\
\mathrm{dr}^{[\mathrm{b}]} \\
\left(\mathbf{6 a}: \mathbf{6 a}^{\prime}\right)\end{array}$} \\
\hline entry & Catalyst & solvent & $\begin{array}{l}\text { Conv } \\
(\%)^{b}\end{array}$ & $\begin{array}{c}\text { Ratio }(\%)^{[b]} \\
\left(\mathbf{6 a}+6 \mathbf{a}^{\prime}\right): \mathbf{6 a}^{\prime \prime}\end{array}$ & \\
\hline 1 & $\mathrm{Rh} / \mathrm{C}$ & HOAc & 70 & $87: 13$ & - \\
\hline $2^{[\mathrm{c}]}$ & $\mathrm{Rh} / \mathrm{C}$ & HOAc & 100 & $0: 100$ & - \\
\hline 3 & $\mathrm{Pt} / \mathrm{C}$ & HOAc & 100 & $0: 100$ & - \\
\hline 4 & $\mathrm{Pd}(\mathrm{OH})_{2} / \mathrm{C}$ & HOAc & $<1$ & - & - \\
\hline 5 & $\mathrm{PtO}_{2}$ & HOAc & 100 & $73: 27$ & 2.68:1 \\
\hline 6 & $\mathrm{PtO}_{2}$ & HFIP & 40 & $71: 29$ & $2.48: 1$ \\
\hline 7 & $\mathrm{PtO}_{2}$ & $\mathrm{MeOH}$ & 17 & $81: 19$ & $1.80: 1$ \\
\hline 8 & $\mathrm{PtO}_{2}$ & $\mathrm{HOAc} / \mathrm{MeOH}(\mathrm{v} / \mathrm{v}=1 / 1)$ & 100 & $73: 27$ & $2.53: 1$ \\
\hline 9 & $\mathrm{PtO}_{2}$ & $\mathrm{HOAc} / \mathrm{MeOH}(\mathrm{v} / \mathrm{v}=1 / 9)$ & 100 & 90:10 & 2.10:1 \\
\hline 10 & $\mathrm{PtO}_{2}$ & $\mathrm{HOAc} / \mathrm{MeOH}(\mathrm{v} / \mathrm{v}=1 / 19)$ & 91 & $93: 7$ & $2.00: 1$ \\
\hline 11 & $\mathrm{PtO}_{2}$ & HOAc/Hexane(v/v = 1/9) & 100 & $82: 18$ & $1.94: 1$ \\
\hline 12 & $\mathrm{PtO}_{2}$ & $\mathrm{TFA} / \mathrm{MeOH}(\mathrm{v} / \mathrm{v}=1 / 9)$ & 85 & $30: 70$ & 1.00:1 \\
\hline
\end{tabular}

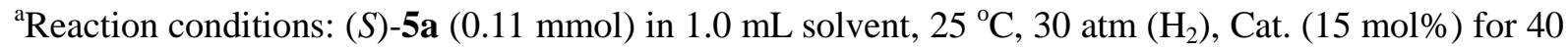
min. ${ }^{\mathrm{b}}$ The conversions and selectivities were determined by ${ }^{1} \mathrm{H}-\mathrm{NMR}$ spectroscopy of the crude reaction mixture. ${ }^{c}$ Reaction time: $2 \mathrm{~h}$.

Scheme S3. Synthesis of optically pure 6a-c

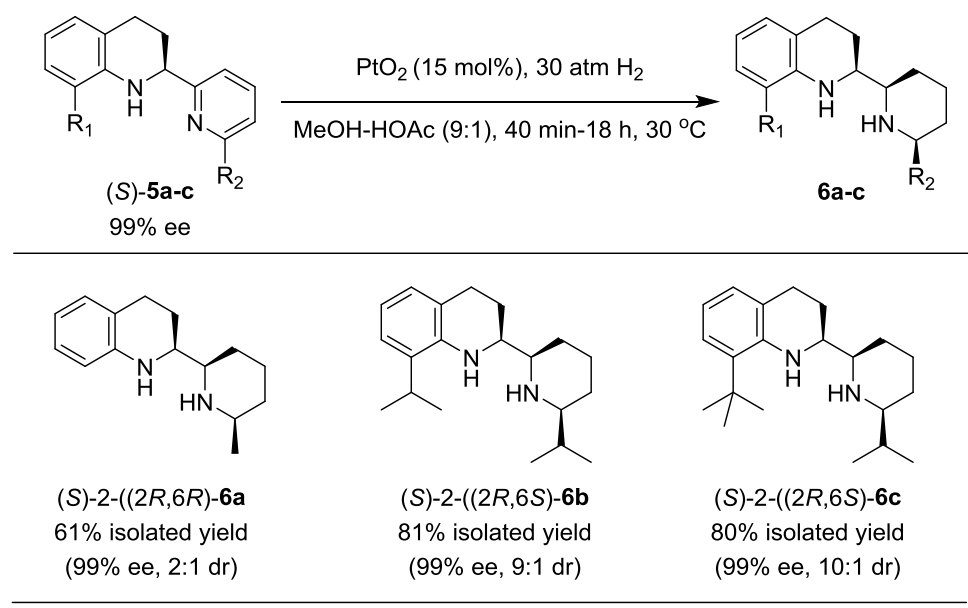


Table S2. Screening of reaction condition for asymmetric intramolecular $\alpha$-arylation. ${ }^{[a]}$<smiles>CC(C(=O)N(C)c1ccccc1Br)c1ccccc1</smiles>

14

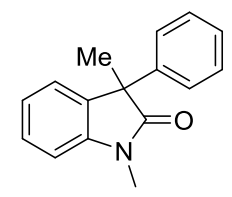

15

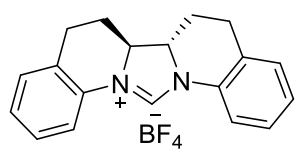

$(2 S, 2 ' S)-3 a$

\begin{tabular}{ccccccc}
\hline entry & solvent & Pd precursors & time (h) & temp $\left({ }^{\circ} \mathrm{C}\right)$ & $\operatorname{conv}(\%)^{[\mathrm{b}]}$ & ee $(\%)^{[\mathrm{c}]}$ \\
\hline 1 & $\mathrm{DME}$ & {$[\mathrm{Pd}(\mathrm{allyl}) \mathrm{Cl}]_{2}$} & 48 & 50 & 16 & 4 \\
2 & $\mathrm{DME}$ & $\mathrm{TMEDA} \cdot \mathrm{PdMe}_{2}$ & 48 & 50 & 12 & 1 \\
3 & $\mathrm{DME}$ & $\mathrm{Pd}(\mathrm{dba})_{2}$ & 48 & 50 & 25 & 7 \\
4 & $\mathrm{DME}$ & $\mathrm{Pd}_{2}(\mathrm{dba})_{3}$ & 48 & 50 & 25 & 8 \\
5 & $\mathrm{DME}$ & $\mathrm{Pd}(\mathrm{OAc})_{2}$ & 48 & 50 & 64 & 24 \\
6 & toluene & $\mathrm{Pd}(\mathrm{OAc})_{2}$ & 48 & 50 & $<5 \%$ & $/$ \\
7 & n-hexane & $\mathrm{Pd}(\mathrm{OAc})_{2}$ & 48 & 50 & $<5 \%$ & $/$ \\
8 & DME & $\mathrm{Pd}(\mathrm{OAc})_{2}$ & 24 & 60 & $>95 \%$ & 24 \\
\hline
\end{tabular}

${ }^{a}$ Substrate $(0.1 \mathrm{mmol})$, Pd precursors $(10.0 \mathrm{~mol} \%), \mathrm{NHC} \cdot \mathrm{BF}_{4}(11.0 \mathrm{~mol} \%), \mathrm{NaO}{ }^{t} \mathrm{Bu}(0.15 \mathrm{mmol})$, in solvent $(2.0 \mathrm{~mL})$ stirred for indicated time. ${ }^{\mathrm{b}}$ The conversions were determined by ${ }^{1} \mathrm{H}$ NMR spectroscopy of the crude reaction mixture. ${ }^{\mathrm{c}}$ The enantiomeric excesses were determined by HPLC with a chiral column. DME $=1,2$-Dimethoxyethane. 


\section{Determination of Molecular Structures}

\subsection{Determination of Molecular Structure of $7 \mathbf{a}^{\prime}$}

To determine the molecular structure, crystals of $(S, R, R)-7 \mathbf{a}^{\prime}$ suitable for X-ray diffraction were obtained after slow evaporation of a concentrated solution in ethyl acetate and n-hexanes at room temperature. The structure in Figure S1 showed that the absolute configuration of $7 \mathbf{a}^{\prime}$ is $(S, R, R)$. [CCDC 1936817] contains the structure and supplementary crystallographic data for $(S, R, R)-7 \mathbf{a}^{\prime}$. These data can be obtained free of charge via www.ccdc.cam.ac.uk/data_request/cif, or by emailing data_request@ccdc.cam.ac.uk.

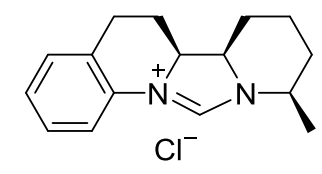

$(S, R, R)-7 a^{\prime}$

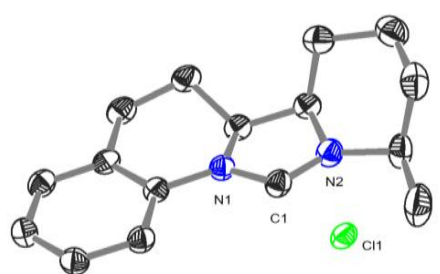

Figure S1. X-ray crystallographic analysis of $7 \mathbf{a}^{\prime}$

\subsection{Determination of Molecular Structure of 8a}

To determine the molecular structure, crystals of 8a suitable for X-ray diffraction were obtained after slow evaporation of a concentrated solution in ethyl acetate at room temperature. The structure in Figure $\mathrm{S} 2$ showed that the absolute configuration of 8a is $(S, S)$. [CCDC 1936782] contains the structure and supplementary crystallographic data for $(S, S)-\mathbf{8 a}$. These data can be obtained free of charge via www.ccdc.cam.ac.uk/data_request/cif, or by emailing data_request@ccdc.cam.ac.uk.

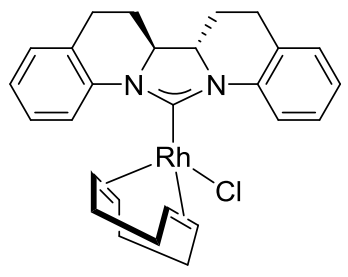

8 a

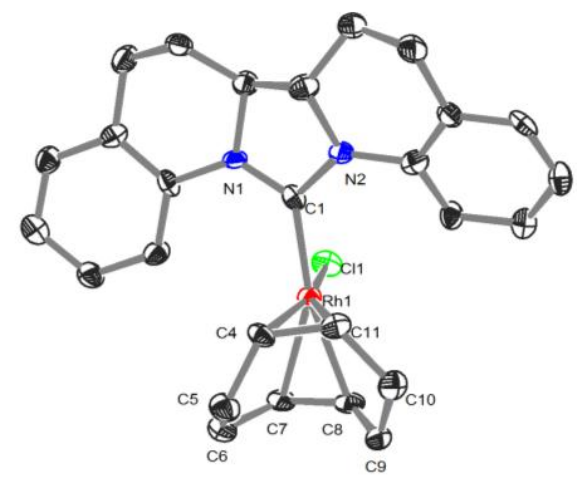

Figure S2. X-ray crystallographic analysis of 8a 


\subsection{Determination of Molecular Structure of $\mathbf{8 b}$}

To determine the molecular structure, crystals of $\mathbf{8 b}$ suitable for X-ray diffraction were obtained after slow evaporation of a concentrated solution in ethyl acetate at room temperature. The structure in Figure $\mathrm{S} 3$ showed that the absolute configuration of $\mathbf{8 b}$ is $(S, S)$. [CCDC 1936783] contains the structure and supplementary crystallographic data for $(S, S)-\mathbf{8 b}$. These data can be obtained free of charge via www.ccdc.cam.ac.uk/data_request/cif, or by emailing data_request@ccdc.cam.ac.uk.
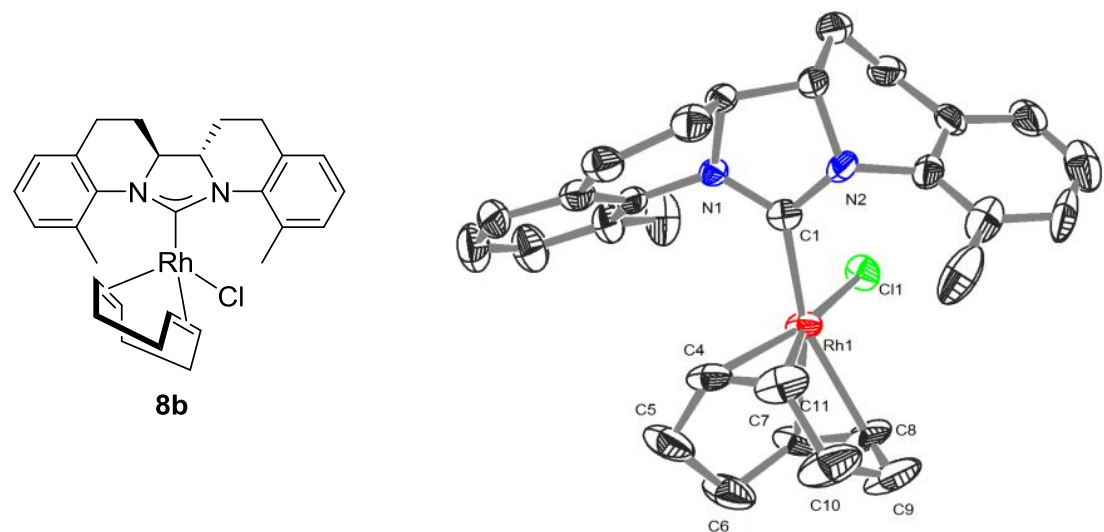

Figure S3. X-ray crystallographic analysis of $\mathbf{8 b}$

\subsection{Determination of Molecular Structure of $\mathbf{8 c}$}

To determine the molecular structure, crystals of $\mathbf{8 c}$ suitable for X-ray diffraction were obtained after slow evaporation of a concentrated solution in ethyl acetate at room temperature. The structure in Figure S4 showed that the absolute configuration of $\mathbf{8 c}$ is $(S, S)$. [CCDC 1736784] contains the structure and supplementary crystallographic data for $(S, S)-8 \mathbf{c}$. These data can be obtained free of charge via www.ccdc.cam.ac.uk/data_request/cif, or by emailingdata_request@ccdc.cam.ac.uk.
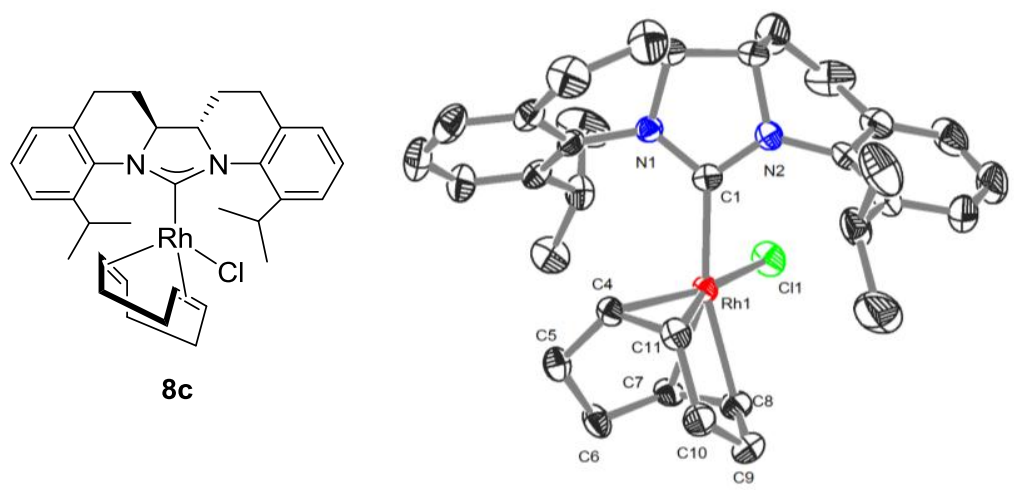

Figure S4. X-ray crystallographic analysis of 8c 


\subsection{Determination of Molecular Structure of 10a}

To determine the molecular structure, crystals of 10a suitable for X-ray diffraction were obtained after slow evaporation of a concentrated solution in ethyl acetate and n-hexanes at room temperature. The structure in Figure S5 showed that the absolute configuration of 10a is $(S, R, R)$. [CCDC 1736786] contains the structure and supplementary crystallographic data for $(S, R, R)$-10a. These data can be obtained free of charge via www.ccdc.cam.ac.uk/data_request/cif, or by emailing data_request@ccdc.cam.ac.uk.
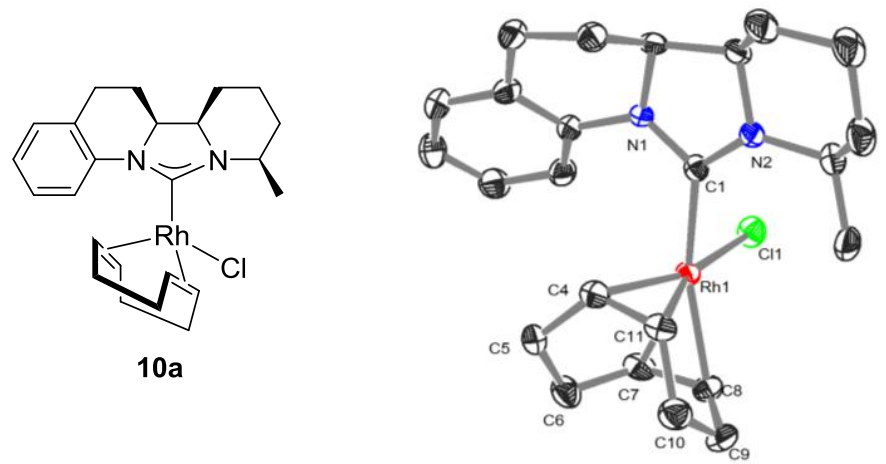

Figure S5. X-ray crystallographic analysis of 10a

\subsection{Determination of Molecular Structure of 10d}

To determine the molecular structure, crystals of $\mathbf{1 0 d}$ suitable for X-ray diffraction were obtained after slow evaporation of a concentrated solution in ethyl acetate at room temperature. The structure in Figure S6 showed that the absolute configuration of 10d is $(S, S, R, R, S)$. [CCDC 1936787] contains the structure and supplementary crystallographic data for $(S, S, R, R, S)$-10d. These data can be obtained free of charge via www.ccdc.cam.ac.uk/data_request/cif, or by emailing data_request@ ccdc.cam.ac.uk.
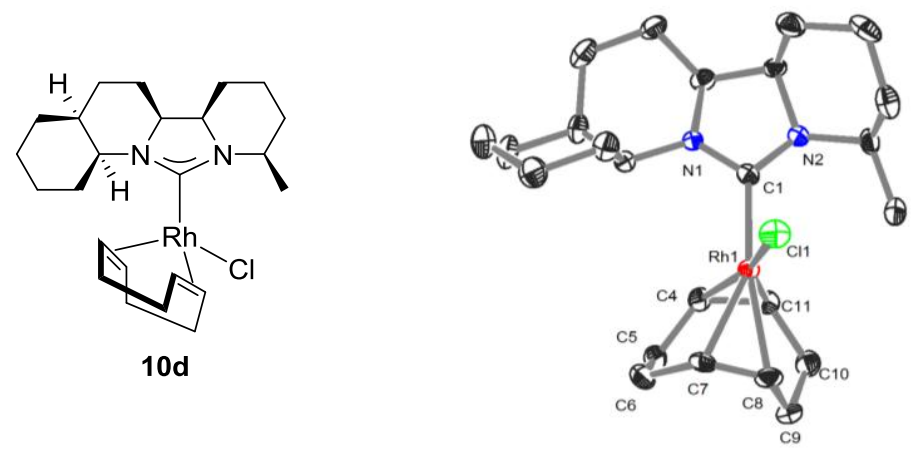

Figure S6. X-ray crystallographic analysis of 10d 


\subsection{Determination of Molecular Structure of $\mathbf{1 2}$}

To determine the molecular structure, crystals of $\mathbf{1 2}$ suitable for X-ray diffraction were obtained after slow evaporation of a concentrated solution in ethyl acetate and n-hexanes at room temperature. The structure in Figure S7 showed that the absolute configuration of $\mathbf{1 2}$ is $(S, S)$. [CCDC 1936785] contains the structure and supplementary crystallographic data for $(S, S)$-12. These data can be obtained free of charge via www.ccdc.cam.ac.uk/data_request/cif, or by emailing data_request@ @ccdc.cam.ac.uk.
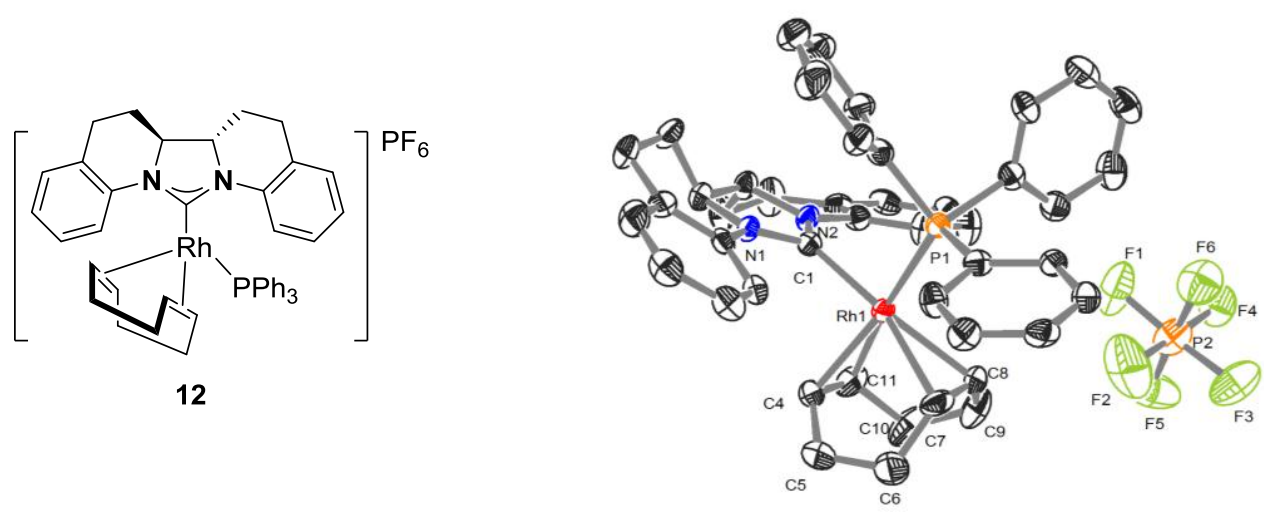

Figure S7. X-ray crystallographic analysis of $\mathbf{1 2}$

\subsection{Determination of Molecular Structure of $\mathbf{1 3}$}

To determine the molecular structure, crystals of $\mathbf{1 3}$ suitable for X-ray diffraction were obtained after slow evaporation of a concentrated solution in ethyl acetate and n-hexanes at room temperature. [CCDC 1936788] contains the structure and supplementary crystallographic data for 13. These data can be obtained free of charge via www.ccdc.cam.ac.uk/data_request/cif, or by emailing data_request@ccdc.cam.ac.uk.
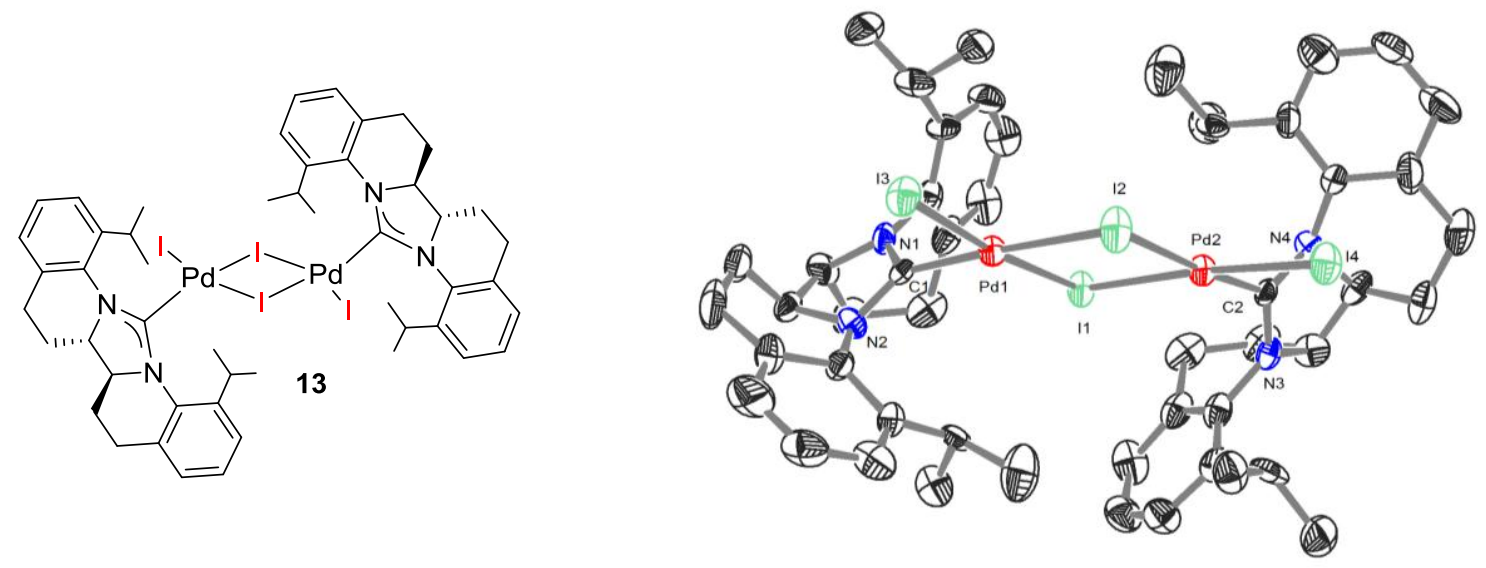

Figure S8. X-ray crystallographic analysis of $\mathbf{1 3}$ 
Table S3. Crystal data and structure refinement for $7 \mathbf{a}^{\prime}$.

Identification code

Empirical formula

Formula weight

Temperature

Wavelength

Crystal system

Space group

Unit cell dimensions

Volume

Z

Density (calculated)

Absorption coefficient

$\mathrm{F}(000)$

Crystal size

Theta range for data collection

Index ranges

Reflections collected

Independent reflections

Completeness to theta $=25.242^{\circ}$

Absorption correction

Max. and min. transmission

Refinement method

Data / restraints / parameters

Goodness-of-fit on $\mathrm{F}^{2}$

Final R indices [I $>2 \operatorname{sigma}(\mathrm{I})]$

$\mathrm{R}$ indices (all data)

Absolute structure parameter

Extinction coefficient

Largest diff. peak and hole
$7 \mathbf{a}^{\prime}$

$\mathrm{C}_{16} \mathrm{H}_{21} \mathrm{ClN}_{2}$

276.80

$173.1500 \mathrm{~K}$

$0.710747 \AA$

Orthorhombic

P 212121

$\begin{array}{ll}\mathrm{a}=7.804(4) \AA & \mathrm{a}=90^{\circ} . \\ \mathrm{b}=10.019(5) \AA & \mathrm{b}=90^{\circ} . \\ \mathrm{c}=18.368(9) \AA & \mathrm{g}=90^{\circ} .\end{array}$

1436.2(12) $\AA^{3}$

4

$1.280 \mathrm{Mg} / \mathrm{m}^{3}$

$0.255 \mathrm{~mm}^{-1}$

592

$0.312 \times 0.207 \times 0.135 \mathrm{~mm}^{3}$

2.218 to $27.477^{\circ}$.

$-10<=\mathrm{h}<=10,-12<=\mathrm{k}<=13,-23<=1<=23$

12313

$3267[\mathrm{R}(\mathrm{int})=0.0483]$

$9990.00 \%$

Semi-empirical from equivalents

1.00000 and 0.88292

Full-matrix least-squares on $\mathrm{F}^{2}$

3267 / 0 / 173

1.145

$\mathrm{R} 1=0.0473, \mathrm{wR} 2=0.0950$

$\mathrm{R} 1=0.0502, \mathrm{wR} 2=0.0964$

$0.01(4)$

$\mathrm{n} / \mathrm{a}$

0.173 and -0.184 e. $\AA^{-3}$ 
Table S4. Crystal data and structure refinement for $\mathbf{8 a}$.

Identification code

Empirical formula

Formula weight

Temperature

Wavelength

Crystal system

Space group

Unit cell dimensions

Volume

$\mathrm{Z}$

Density (calculated)

Absorption coefficient

$\mathrm{F}(000)$

Theta range for data collection

Index ranges

Reflections collected

Independent reflections

Completeness to theta $=25.242^{\circ}$

Absorption correction

Refinement method

Data / restraints / parameters

Goodness-of-fit on $\mathrm{F}^{2}$

Final $\mathrm{R}$ indices [I $>2 \operatorname{sigma}(\mathrm{I})]$

$\mathrm{R}$ indices (all data)

Absolute structure parameter

Extinction coefficient

Largest diff. peak and hole
$8 \mathbf{a}$

$\mathrm{C}_{27} \mathrm{H}_{30} \mathrm{Cl} \mathrm{N} \mathrm{Nh}_{2}$

520.89

293(2) K

$0.71073 \AA$

Monoclinic

P 1211

$\mathrm{a}=11.528(2) \AA$

$\mathrm{a}=90^{\circ}$.

$\mathrm{b}=8.9796(18) \AA$

$\mathrm{b}=100.38(3)^{\circ}$.

$c=21.706(4) \AA$

$\mathrm{g}=90^{\circ}$.

4

$1.565 \mathrm{mg} / \mathrm{m}^{3}$

$0.912 \mathrm{~mm}^{-1}$

1072

1.796 to $27.489^{\circ}$.

$-14<=\mathrm{h}<=14,-11<=\mathrm{k}<=11,-27<=\mathrm{l}<=28$

25745

$10031[\mathrm{R}$ (int) $=0.0477]$

$99.6 \%$

None

Full-matrix least-squares on $\mathrm{F}^{2}$

$10031 / 1 / 559$

1.086

$\mathrm{R} 1=0.0457, \mathrm{wR} 2=0.0824$

$\mathrm{R} 1=0.0509, \mathrm{wR} 2=0.0853$

0.04(3)

$\mathrm{n} / \mathrm{a}$

0.434 and -0.454 e. $\AA^{-3}$ 
Table S5. Crystal data and structure refinement for $(\mathbf{8 b})_{2}-\mathbf{C H}_{3} \mathrm{COOEt}$.

Identification code

Empirical formula

Formula weight

Temperature

Wavelength

Crystal system

Space group

Unit cell dimensions

Volume

Z

Density (calculated)

Absorption coefficient

$\mathrm{F}(000)$

Crystal size

Theta range for data collection

Index ranges

Reflections collected

Independent reflections

Completeness to theta $=25.242^{\circ}$

Absorption correction

Max. and min. transmission

Refinement method

Data / restraints / parameters

Goodness-of-fit on $\mathrm{F}^{2}$

Final R indices [I $>2 \operatorname{sigma}(\mathrm{I})]$

$\mathrm{R}$ indices (all data)

Absolute structure parameter

Extinction coefficient

Largest diff. peak and hole

\section{$(8 b)_{2}-\mathrm{CH}_{3} \mathrm{COOEt}$}

$\mathrm{C}_{62} \mathrm{H}_{76} \mathrm{Cl}_{2} \mathrm{~N}_{4} \mathrm{O}_{2} \mathrm{Rh}_{2}$

1185.98

$173.15 \mathrm{~K}$

$0.71073 \AA$

Monoclinic

P 1211

$\mathrm{a}=11.474(3) \AA \quad \mathrm{a}=90^{\circ}$.

$\mathrm{b}=10.161(2) \AA \quad \mathrm{b}=91.569(3)^{\circ}$.

$\mathrm{c}=23.799(6) \AA \quad \mathrm{g}=90^{\circ}$.

$2773.5(11) \AA^{3}$

2

$1.420 \mathrm{mg} / \mathrm{m}^{3}$

$0.738 \mathrm{~mm}^{-1}$

1232

$0.256 \times 0.031 \times 0.027 \mathrm{~mm}^{3}$

1.712 to $27.498^{\circ}$.

$-14<=\mathrm{h}<=14,-13<=\mathrm{k}<=13,-30<=1<=30$

31924

$12300[\mathrm{R}$ (int) $=0.0668]$

$99.9 \%$

Semi-empirical from equivalents

1.00000 and 0.78856

Full-matrix least-squares on $\mathrm{F}^{2}$

12300 / 43 / 665

1.099

$\mathrm{R} 1=0.0588, \mathrm{wR} 2=0.0915$

$\mathrm{R} 1=0.0656, \mathrm{wR} 2=0.0953$

$0.01(2)$

$\mathrm{n} / \mathrm{a}$

0.542 and -0.710 e. $\AA^{-3}$ 
Table S6. Crystal data and structure refinement for $\mathbf{8 c}$.

Identification code

Empirical formula

Formula weight

Temperature

Wavelength

Crystal system

Space group

Unit cell dimensions

Volume

$\mathrm{Z}$

Density (calculated)

Absorption coefficient

$\mathrm{F}(000)$

Crystal size

Theta range for data collection

Index ranges

Reflections collected

Independent reflections

Completeness to theta $=25.242^{\circ}$

Absorption correction

Max. and min. transmission

Refinement method

Data / restraints / parameters

Goodness-of-fit on $\mathrm{F}^{2}$

Final $\mathrm{R}$ indices [I $>2 \operatorname{sigma}(\mathrm{I})]$

$\mathrm{R}$ indices (all data)

Absolute structure parameter

Extinction coefficient

Largest diff. peak and hole
$8 c$

$\mathrm{C}_{33} \mathrm{H}_{42} \mathrm{ClN}_{2} \mathrm{Rh}$

605.04

$173.15 \mathrm{~K}$

$0.71073 \AA$

Orthorhombic

$\mathrm{P} 2{ }_{1} 2_{1} 2_{1}$

$\mathrm{a}=12.6364(6) \AA$

$\mathrm{a}=90^{\circ}$.

$\mathrm{b}=14.7003(7) \AA$

$\mathrm{b}=90^{\circ}$.

$\mathrm{c}=15.3650(9) \AA$

$\mathrm{g}=90^{\circ}$.

4

$1.408 \mathrm{mg} / \mathrm{m}^{3}$

$0.717 \mathrm{~mm}^{-1}$

1264

$0.564 \times 0.189 \times 0.147 \mathrm{~mm}^{3}$

2.992 to $27.487^{\circ}$.

$-16<=\mathrm{h}<=16,-19<=\mathrm{k}<=19,-19<=\mathrm{l}<=19$

19418

$6347[\mathrm{R}(\mathrm{int})=0.0570]$

$99.7 \%$

Semi-empirical from equivalents

1.00000 and 0.70764

Full-matrix least-squares on $\mathrm{F}^{2}$

6347 / 0 / 338

1.070

$\mathrm{R} 1=0.0408, \mathrm{wR} 2=0.0854$

$\mathrm{R} 1=0.0420, \mathrm{wR} 2=0.0862$

$0.01(2)$

$\mathrm{n} / \mathrm{a}$

0.595 and -0.823 e. $\AA^{-3}$ 
Table S7. Crystal data and structure refinement for $\mathbf{1 0 a}$

Identification code

Empirical formula

Formula weight

Temperature

Wavelength

Crystal system

Space group

Unit cell dimensions

Volume

$\mathrm{Z}$

Density (calculated)

Absorption coefficient

$\mathrm{F}(000)$

Theta range for data collection

Index ranges

Reflections collected

Independent reflections

Completeness to theta $=25.242^{\circ}$

Absorption correction

Max. and min. transmission

Refinement method

Data / restraints / parameters

Goodness-of-fit on $\mathrm{F}^{2}$

Final $\mathrm{R}$ indices [I $>2 \operatorname{sigma}(\mathrm{I})]$

$\mathrm{R}$ indices (all data)

Absolute structure parameter

Extinction coefficient

Largest diff. peak and hole

\section{$10 a$}

$\mathrm{C}_{24} \mathrm{H}_{32} \mathrm{ClN}_{2} \mathrm{Rh}$

486.87

173(2) K

$0.71073 \AA$

Orthorhombic

P 212121

$\begin{array}{ll}\mathrm{a}=9.1756(10) \AA & \mathrm{a}=90^{\circ} . \\ \mathrm{b}=12.5447(14) \AA & \mathrm{b}=90^{\circ} . \\ \mathrm{c}=18.664(2) \AA & \mathrm{g}=90^{\circ} .\end{array}$

2148.3(4) $\AA^{3}$

4

$1.505 \mathrm{mg} / \mathrm{m}^{3}$

$0.932 \mathrm{~mm}^{-1}$

1008

1.956 to $27.477^{\circ}$.

$-11<=\mathrm{h}<=11,-16<=\mathrm{k}<=16,-24<=1<=24$

13462

$4835[\mathrm{R}(\mathrm{int})=0.0360]$

$99.1 \%$

Semi-empirical from equivalents

1.0000 and 0.8667

Full-matrix least-squares on $\mathrm{F}^{2}$

4835 / 0 / 254

1.084

$\mathrm{R} 1=0.0281, \mathrm{wR} 2=0.0624$

$\mathrm{R} 1=0.0299, \mathrm{wR} 2=0.0637$

$-0.021(18)$

$\mathrm{n} / \mathrm{a}$

0.315 and -0.577 e. $\AA^{-3}$ 
Table S8. Crystal data and structure refinement for $\mathbf{1 0 d}$.

Identification code

Empirical formula

Formula weight

Temperature

Wavelength

Crystal system

Space group

Unit cell dimensions

Volume

Z

Density (calculated)

Absorption coefficient

$\mathrm{F}(000)$

Theta range for data collection

Index ranges

Reflections collected

Independent reflections

Completeness to theta $=25.242^{\circ}$

Absorption correction

Refinement method

Data / restraints / parameters

Goodness-of-fit on $\mathrm{F}^{2}$

Final R indices [I $>2 \operatorname{sigma}(\mathrm{I})]$

$\mathrm{R}$ indices (all data)

Absolute structure parameter

Extinction coefficient

Largest diff. peak and hole 10d

$\mathrm{C}_{24} \mathrm{H}_{38} \mathrm{ClN}_{2} \mathrm{Rh}$

492.92

293(2) K

$0.71073 \AA$

Orthorhombic

P 212121

$\begin{array}{ll}\mathrm{a}=10.810(2) \AA & \mathrm{a}=90^{\circ} . \\ \mathrm{b}=11.709(2) \AA & \mathrm{b}=90^{\circ} . \\ \mathrm{c}=17.758(4) \AA & \mathrm{g}=90^{\circ} .\end{array}$

2247.8(8) $\AA^{3}$

4

$1.457 \mathrm{mg} / \mathrm{m}^{3}$

$0.891 \mathrm{~mm}^{-1}$

1032

2.083 to $27.481^{\circ}$.

$-14<=\mathrm{h}<=14,-9<=\mathrm{k}<=15,-23<=1<=15$

8869

$5036[\mathrm{R}(\mathrm{int})=0.0329]$

$99.7 \%$

None

Full-matrix least-squares on $\mathrm{F}^{2}$

$5036 / 0 / 254$

1.048

$\mathrm{R} 1=0.0328, \mathrm{wR} 2=0.0678$

$\mathrm{R} 1=0.0348, \mathrm{wR} 2=0.0692$

$-0.01(2)$

$\mathrm{n} / \mathrm{a}$

0.478 and -0.426 e. $\AA^{-3}$ 
Table S9. Crystal data and structure refinement for $\mathbf{1 2} \cdot \mathbf{C H}_{\mathbf{2}} \mathbf{C l}_{\mathbf{2}}$

Identification code

Empirical formula

Formula weight

Temperature

Wavelength

Crystal system

Space group

Unit cell dimensions

Volume

Z

Density (calculated)

Absorption coefficient

$\mathrm{F}(000)$

Theta range for data collection

Index ranges

Reflections collected

Independent reflections

Completeness to theta $=25.242^{\circ}$

Absorption correction

Refinement method

Data / restraints / parameters

Goodness-of-fit on $\mathrm{F}^{2}$

Final $\mathrm{R}$ indices [I $>2 \operatorname{sigma}(\mathrm{I})]$

$\mathrm{R}$ indices (all data)

Absolute structure parameter

Extinction coefficient

Largest diff. peak and hole

\section{$12 \cdot \mathrm{CH}_{2} \mathrm{Cl}_{2}$}

$\mathrm{C}_{46} \mathrm{H}_{47} \mathrm{Cl}_{2} \mathrm{~F}_{6} \mathrm{~N}_{2} \mathrm{P}_{2} \mathrm{Rh}$

977.60

293(2) K

$0.71073 \AA$

Monoclinic

P 1211

$\begin{array}{ll}\mathrm{a}=12.7457(3) \AA & \mathrm{a}=90^{\circ} . \\ \mathrm{b}=15.5358(3) \AA & \mathrm{b}=102.568(2)^{\circ} . \\ \mathrm{c}=22.0173(5) \AA & \mathrm{g}=90^{\circ} .\end{array}$

4255.30(16) $\AA^{3}$

4

$1.526 \mathrm{mg} / \mathrm{m}^{3}$

$0.665 \mathrm{~mm}^{-1}$

2000

1.704 to $27.499^{\circ}$.

$-16<=\mathrm{h}<=16,-20<=\mathrm{k}<=20,-28<=1<=28$

55177

19524 [R(int) $=0.0463]$

$99.9 \%$

None

Full-matrix least-squares on $\mathrm{F}^{2}$

19524 / 1 / 1063

1.039

$\mathrm{R} 1=0.0462, \mathrm{wR} 2=0.0963$

$\mathrm{R} 1=0.0583, \mathrm{wR} 2=0.1030$

$-0.042(10)$

$\mathrm{n} / \mathrm{a}$

1.069 and -0.455 e. $\AA^{-3}$ 
Table S10. Crystal data and structure refinement for 13.

Identification code

Empirical formula

Formula weight

Temperature

Wavelength

Crystal system

Space group

Unit cell dimensions

Volume

Z

Density (calculated)

Absorption coefficient

$\mathrm{F}(000)$

Theta range for data collection

Index ranges

Reflections collected

Independent reflections

Completeness to theta $=24.994^{\circ}$

Absorption correction

Refinement method

Data / restraints / parameters

Goodness-of-fit on $\mathrm{F}^{2}$

Final R indices [I $>2 \operatorname{sigma}(\mathrm{I})]$

$\mathrm{R}$ indices (all data)

Absolute structure parameter

Extinction coefficient

Largest diff. peak and hole
13

$\mathrm{C}_{50} \mathrm{H}_{56} \mathrm{I}_{4} \mathrm{~N}_{4} \mathrm{Pd}_{2}$

1433.38

293(2) K

$0.71073 \AA$

Monoclinic

C 121

$\begin{array}{ll}\mathrm{a}=24.1544(16) \AA & \mathrm{a}=90^{\circ} . \\ \mathrm{b}=10.1485(4) \AA & \mathrm{b}=104.452(7)^{\circ} . \\ \mathrm{c}=12.0584(9) \AA & \mathrm{g}=90^{\circ} .\end{array}$

2862.4(3) $\AA^{3}$

2

$1.663 \mathrm{mg} / \mathrm{m}^{3}$

$2.818 \mathrm{~mm}^{-1}$

1376

2.659 to $24.994^{\circ}$.

$-22<=\mathrm{h}<=28,-11<=\mathrm{k}<=12,-14<=\mathrm{l}<=13$

8883

$4747[\mathrm{R}(\mathrm{int})=0.0509]$

$99.6 \%$

None

Full-matrix least-squares on $\mathrm{F}^{2}$

4747 / 13 / 276

1.007

$\mathrm{R} 1=0.0534, \mathrm{wR} 2=0.0952$

$\mathrm{R} 1=0.0688, \mathrm{wR} 2=0.1014$

$0.00(3)$

$\mathrm{n} / \mathrm{a}$

0.976 and -0.585 e. $\AA^{-3}$ 


\section{General procedure for the synthesis of substrates}

\subsection{Synthesis of 2,2'-Bisquinoline Derivatives 1a-g.}

2,2'-Bisquinoline Derivatives 1 could be synthesized according to the previously reported procedures. ${ }^{[2-3]}$ Among them, 1a-1e are the known compounds. ${ }^{[2 b]} \mathbf{1 f}$ and $\mathbf{1 g}$ are new compounds. They were synthesized by following steps. Moreover, the corresponding 2-aminobenzyl aldehyde and 2-acetylquinoline intermediates were synthesized according to the previously reported procedures. ${ }^{[2-3]}$

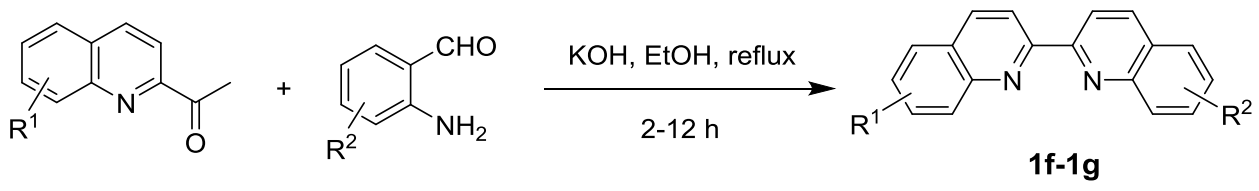

Typical procedure: To a suspension of 1-(8-isopropylquinolin-2-yl)ethanone $(0.85 \mathrm{~g}, 3.96$ mmol) and 2-aminobenzaldehyde $(0.40 \mathrm{~g}, 3.30 \mathrm{mmol})$ in $\mathrm{EtOH}(20 \mathrm{~mL})$ was added $\mathrm{KOH}(37$ $\mathrm{mg}, 0.66 \mathrm{mmol})$. Then, the mixture was heated at reflux and stirred for $2 \mathrm{~h}$. The resulting mixture was cooled and the solvent was evaporated in vacuo. The residue was purified by column chromatography (eluent:ethyl acetate $/$ petroleum ether $=1 / 300, v / v$ ) affording 8-isopropyl-2,2'-biquinoline $\mathbf{1 f}$ as a white solid (0.82 g, 83\% yield). The analytical data of the products are summarized below.

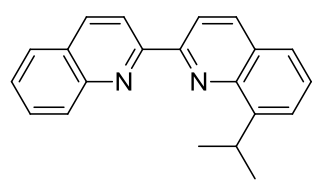

1f (New compound): White solid, $822 \mathrm{mg}, 83 \%$ yield, m.p. $116-118{ }^{\circ} \mathrm{C}$. ${ }^{1} \mathrm{H}$ NMR $\left(500 \mathrm{MHz}, \mathrm{CDCl}_{3}\right): \delta(\mathrm{ppm}) 8.91(\mathrm{~d}, J=8.5 \mathrm{~Hz}, 1 \mathrm{H}), 8.86(\mathrm{~d}, J$ $=8.5 \mathrm{~Hz}, 1 \mathrm{H}), 8.31(\mathrm{t}, J=8.5 \mathrm{~Hz}, 2 \mathrm{H}), 8.23(\mathrm{~d}, J=8.5 \mathrm{~Hz}, 1 \mathrm{H}), 7.89(\mathrm{~d}$, $J=8.0 \mathrm{~Hz}, 1 \mathrm{H}), 7.77-7.74(\mathrm{~m}, 2 \mathrm{H}), 7.72(\mathrm{~d}, J=8.0 \mathrm{~Hz}, 1 \mathrm{H}), 7.65(\mathrm{~d}, J=7.0 \mathrm{~Hz}, 1 \mathrm{H})$, 7.59-7.52 (m, 2H), 4.60-4.51 (m, 1H), $1.50(\mathrm{~d}, J=7.0 \mathrm{~Hz}, 6 \mathrm{H}) ;{ }^{13} \mathrm{C}$ NMR $\left(125 \mathrm{MHz}, \mathrm{CDCl}_{3}\right)$ : $\delta(\mathrm{ppm}) 156.9,154.6,148.1,148.0,145.7,137.3,136.7,130.0,129.6,128.7,128.6,127.8$, 127.1, 127.0, 125.6, 125.5, 119.7, 118.9, 28.0, 23.7. HRMS (ESI) m/z: $[\mathrm{M}+\mathrm{H}]^{+}$Calcd for $\mathrm{C}_{21} \mathrm{H}_{19} \mathrm{~N}_{2}$ 299.15428; Found 299.15409. 


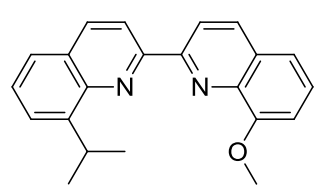

$\mathbf{1 g}$ (New compound): Following the similar synthetic procedure of $\mathbf{1 f}$ in $7.6 \mathrm{mmol}$ scale for $12 \mathrm{~h}$, The crude product was purified by column chromatography (eluent: ethyl acetate/petroleum ether $=1 / 200, v / v$ ) affording 8-isopropyl-8-methoxy-2,2'-biquinoline $1 \mathrm{~g}$ as Pale yellow solid (1.9 g, $76 \%$ yield). m.p. $220-225{ }^{\circ} \mathrm{C} .{ }^{1} \mathrm{H}$ NMR $\left(500 \mathrm{MHz}, \mathrm{CDCl}_{3}\right): \delta(\mathrm{ppm}) 8.96(\mathrm{~d}, J=8.5 \mathrm{~Hz}, 1 \mathrm{H}), 8.90(\mathrm{~d}, J=$ $8.5 \mathrm{~Hz}, 1 \mathrm{H}), 8.30\left(\mathrm{dd}, J_{1}=8.5 \mathrm{~Hz}, J_{2}=5.5 \mathrm{~Hz}, 2 \mathrm{H}\right), 7.71\left(\mathrm{dd}, J_{l}=8.0 \mathrm{~Hz}, J_{2}=1.0 \mathrm{~Hz}, 2 \mathrm{H}\right)$, $7.63(\mathrm{~d}, J=7.0 \mathrm{~Hz}, 1 \mathrm{H}), 7.54-7.46(\mathrm{~m}, 3 \mathrm{H}), 7.11\left(\mathrm{dd}, J_{l}=7.5 \mathrm{~Hz}, J_{2}=1.0 \mathrm{~Hz}, 1 \mathrm{H}\right), 4.59-4.51$ $(\mathrm{m}, 1 \mathrm{H}), 4.17(\mathrm{~s}, 3 \mathrm{H}), 1.49(\mathrm{~d}, J=6.5 \mathrm{~Hz}, 6 \mathrm{H}) ;{ }^{13} \mathrm{C} \mathrm{NMR}\left(125 \mathrm{MHz}, \mathrm{CDCl}_{3}\right): \delta(\mathrm{ppm}) 155.8$, $154.6,147.9,145.7,139.8,137.2,136.8,129.7,128.7,127.2,127.0,125.5,125.4,120.2$, 119.8, 119.2, 108.2, 56.4, 27.9, 23.7. HRMS (ESI) $\mathrm{m} / \mathrm{z}:[\mathrm{M}+\mathrm{H}]^{+}$Calcd for $\mathrm{C}_{22} \mathrm{H}_{21} \mathrm{ON}_{2}$ 329.16484; Found 329.16462.

\subsection{Synthesis of 2-(pyridin-2-yl)quinoline Derivatives 4a-c.}

The corresponding 2-aminobenzyl aldehyde and 2-acetylpyridine intermediates were synthesized according to the previously reported procedures. ${ }^{[4]} \mathbf{4 a}$ and $\mathbf{4 c}$ are the known compounds. ${ }^{[4]}$

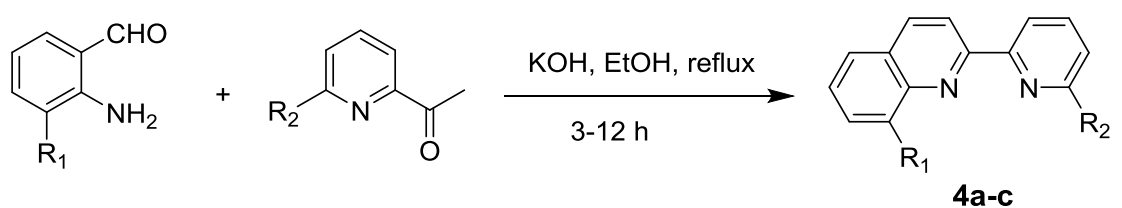

Typical procedure: To a stirred solution of 2-aminobenzyl aldehyde in EtOH (1.0 M), 2-acetylpyridine (1.2 eq) and $\mathrm{KOH}(0.2 \mathrm{eq})$ were added. The reaction mixture was stirred at reflux for 3-12 h. After cooling to room temperature, the mixture was evaporated under reduced pressure. The residue was purified by silica gel column chromatography (eluent: ethyl acetate / petroleum ether $=1 / 50 \sim 1 / 200$ ) to give 4a-c as white solid or colorless oil. The analytical data of the products are summarized below.

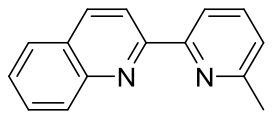

4a (Known compound, see: Liu, Y.; Chen, F.; He, Y.-M.; Li, C.; Fan, Q.-H.

Org. Biomol. Chem. 2019, 17, 5099-5105). white solid, m.p. $96-98{ }^{\circ}$ C. 2.3 g, 
92\% yield; ${ }^{1} \mathrm{H}$ NMR $\left(300 \mathrm{MHz}, \mathrm{CDCl}_{3}\right): \delta(\mathrm{ppm}) 8.59(\mathrm{~d}, J=8.7 \mathrm{~Hz}, 1 \mathrm{H}), 8.44(\mathrm{~d}, J=7.8 \mathrm{~Hz}$, 1H), $8.25(\mathrm{~d}, J=8.4 \mathrm{~Hz}, 1 \mathrm{H}), 8.17(\mathrm{~d}, J=8.7 \mathrm{~Hz}, 1 \mathrm{H}), 7.83(\mathrm{~d}, J=8.1 \mathrm{~Hz}, 1 \mathrm{H}), 7.77-7.69(\mathrm{~m}$, 2H), 7.55-7.50 (m, 1H), $7.20(\mathrm{~d}, J=7.5 \mathrm{~Hz}, 1 \mathrm{H}), 2.67(\mathrm{~s}, 3 \mathrm{H}) ;{ }^{13} \mathrm{C}$ NMR $\left(75 \mathrm{MHz}, \mathrm{CDCl}_{3}\right): \delta$ (ppm) 158.0, 156.7, 155.9, 148.1, 137.2, 136.8, 129.9, 129.6, 128.3, 127.7, 126.7, 123.7, $119.3,118.9,24.8$.

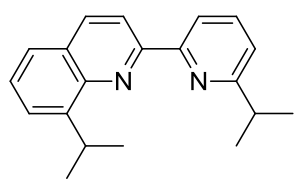

4b (New compound): colorless oil, $800 \mathrm{mg}$, 64\% yield; ${ }^{1} \mathrm{H}$ NMR (300 MHz, $\left.\mathrm{CDCl}_{3}\right): \delta(\mathrm{ppm}) 8.68(\mathrm{~d}, J=8.4 \mathrm{~Hz}, 1 \mathrm{H}), 8.53(\mathrm{~d}, J=7.8 \mathrm{~Hz}$, $1 \mathrm{H}), 8.22(\mathrm{~d}, J=8.7 \mathrm{~Hz}, 1 \mathrm{H}), 7.77(\mathrm{t}, J=7.8 \mathrm{~Hz}, 1 \mathrm{H}), 7.66(\mathrm{~d}, J=8.1 \mathrm{~Hz}$, $1 \mathrm{H}), 7.59(\mathrm{~d}, J=6.9 \mathrm{~Hz}, 1 \mathrm{H}), 7.48(\mathrm{t}, J=7.7 \mathrm{~Hz}, 1 \mathrm{H}), 7.21(\mathrm{~d}, J=7.8 \mathrm{~Hz}, 1 \mathrm{H}), 4.56-4.42(\mathrm{~m}$, $1 \mathrm{H}), 3.25-3.11(\mathrm{~m}, 1 \mathrm{H}), 1.46(\mathrm{~d}, J=6.9 \mathrm{~Hz}, 6 \mathrm{H}), 1.40(\mathrm{~d}, J=6.9 \mathrm{~Hz}, 6 \mathrm{H}) ;{ }^{13} \mathrm{C} \mathrm{NMR}(75 \mathrm{MHz}$, $\left.\mathrm{CDCl}_{3}\right): \delta$ (ppm) 166.6, 156.0, 155.1, 147.8, 145.7, 137.2, 137.0, 128.4, 126.6, 125.5, 125.2, 120.9, 119.0, 118.7, 36.5, 27.9, 23.7, 22.9. HRMS (ESI) m/z: $[\mathrm{M}+\mathrm{H}]^{+}$Calcd for $\mathrm{C}_{20} \mathrm{H}_{23} \mathrm{~N}_{2}$ 291.18558; Found 291.18481.

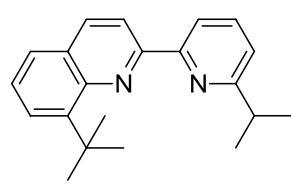

4c (Known compound, see: Liu, Y.; Chen, F.; He, Y.-M.; Li, C.; Fan, Q.-H. Org. Biomol. Chem. 2019, 17, 5099-5105). colorless oil, 1.2 g, 90\% yield. ${ }^{1} \mathrm{H}$ NMR (400 MHz, $\left.\mathrm{CDCl}_{3}\right): \delta(\mathrm{ppm}) 8.67(\mathrm{~d}, J=8.8 \mathrm{~Hz}, 1 \mathrm{H}), 8.48-8.46$ (m, 1H), $8.21(\mathrm{~d}, J=8.8 \mathrm{~Hz}, 1 \mathrm{H}), 7.78(\mathrm{t}, J=7.8 \mathrm{~Hz}, 1 \mathrm{H}), 7.68-7.65(\mathrm{~m}, 2 \mathrm{H}), 7.43(\mathrm{t}, J=7.8$ $\mathrm{Hz}, 1 \mathrm{H}), 7.20$ (d, $J=7.6 \mathrm{~Hz}, 1 \mathrm{H}), 3.21-3.14(\mathrm{~m}, 1 \mathrm{H}), 1.77$ (s, 9H), $1.40(\mathrm{~d}, J=7.2 \mathrm{~Hz}, 6 \mathrm{H})$; ${ }^{13} \mathrm{C}$ NMR (100 MHz, $\left.\mathrm{CDCl}_{3}\right): \delta$ (ppm) 166.6, 156.0, 153.9, 148.3, 146.7, 137.4, 137.3, 129.3, 126.7, 126.2, 126.2, 126.2, 120.9, 119.0, 118.1,36.8, 36.5, 31.5, 22.9. HRMS (ESI) m/z: $[\mathrm{M}+\mathrm{H}]^{+}$Calcd for $\mathrm{C}_{21} \mathrm{H}_{25} \mathrm{~N}_{2}$ 305.20123; Found 305.20102.

\subsection{General procedure for scale-up of asymmetric hydrogenation}

The chiral hydrogenated products 2 could be synthesized according to our reported procedures. ${ }^{[2 \mathrm{~b}]}$ Among them, $(2 S, 2 ' S)-\mathbf{2 a - 2 e}$ are the known compounds. 

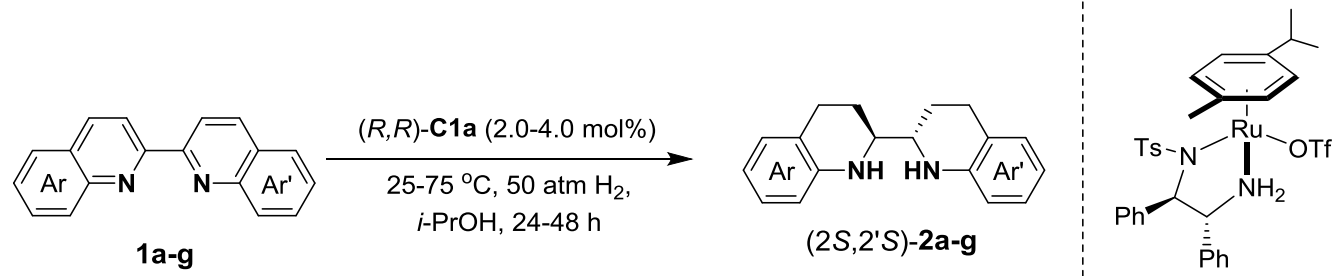

$(R, R)-\mathbf{C 1 a}$

Typical procedure: In a $50 \mathrm{~mL}$ glass-lined stainless steel reactor with a magnetic stirring bar was charged with Ru-catalyst $(R, R)-\mathbf{C 1 a}(12 \mathrm{mg}, 0.016 \mathrm{mmol}), \mathbf{1 a}(200 \mathrm{mg}, 0.78 \mathrm{mmol})$ and $i$-PrOH $(10 \mathrm{~mL})$. The autoclave was closed, and the final pressure of the hydrogen gas was adjusted to $50 \mathrm{~atm}$ after purging the autoclave with hydrogen gas several times. The reaction mixture was stirred at $25{ }^{\circ} \mathrm{C}$ for $24 \mathrm{~h}$. Then the hydrogen gas was carefully released and the conversion was determined by ${ }^{1} \mathrm{H}$ NMR spectroscopy after the solvent was evaporated under reduced pressure. After concentration, the diastereoisomer was purified under slow flowing by silica gel column chromatography (eluent: petroleum ether/ethyl acetate $=300 / 1-50 / 1, v / v$ ) to give the one of the pure isomer $\left(2 S, 2^{\prime} S\right)$-2a as pale yellow oil (184 mg, $89 \%$ yield, $99 \%$ ee).

(2S,2'S)-2a (Known compound, see: Ma, W.; Zhang, J.; Xu, C.; Chen, F.; He, Y.-M.; Fan, Q.-H. Angew. Chem. Int. Ed. 2016, 55, 12891-12894). $184 \mathrm{mg}, 89 \%$ yield, 99\% ee. ${ }^{1} \mathrm{H}$ NMR (300 MHz, $\left.\mathrm{CDCl}_{3}\right): \delta$ (ppm) 7.01-6.96 (m, 4H), 6.66-6.55 (m, 4H), 4.13 (brs, 2H), 3.30-3.27 (m, 2H), 2.86-2.71 (m, 4H), 2.04-1.95 (m, 2H), 1.89-1.80 (m, 2H); ${ }^{13} \mathrm{C}$ NMR (125 MHz, $\left.\mathrm{CDCl}_{3}\right): \delta$ (ppm) 144.1, 129.3, 127.0, 121.8, 117.6, 115.0, 54.9, 25.9, 24.3 .

The enantiomeric excess was determined by HPLC on Chiralcel AD-H column (n-hexane : isopropanol $=80: 20$, flowing rate $=1.0 \mathrm{~mL} / \mathrm{min}, 20^{\circ} \mathrm{C}$, $\mathrm{UV}$ detection at $\lambda=254$ $\mathrm{nm}), \mathrm{t}_{\mathrm{R} 1}=8.9 \min ($ minor $), \mathrm{t}_{\mathrm{R} 2}=13.0 \mathrm{~min}$ (major).

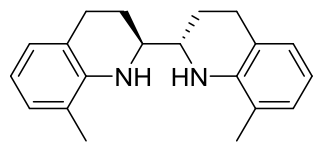

(2S,2'S)-2b (Known compound, see: Ma, W.; Zhang, J.; Xu, C.; Chen, F.;

He, Y.-M.; Fan, Q.-H. Angew. Chem. Int. Ed. 2016, 55, 12891-12894). Following the similar synthetic procedure of $(2 S, 2 ' S)-\mathbf{2 a}$ in $0.7 \mathrm{mmol}$ scale with $4.0 \mathrm{~mol} \%(R, R)-\mathbf{C 1 a}$ at $75{ }^{\circ} \mathrm{C}$ for $24 \mathrm{~h}$. The diastereoisomer was purified under 
slow flowing by silica gel column chromatography (eluent: petroleum ether/ethyl acetate = $300 / 1-100 / 1, v / v)$ to give the one of the pure isomer $\left(2 S, 2^{\prime} S\right)-\mathbf{2 b}$ as pale yellow oil $(102 \mathrm{mg}, 50 \%$ yield, 99\% ee). ${ }^{1} \mathrm{H}$ NMR (300 MHz, $\left.\mathrm{CDCl}_{3}\right): \delta(\mathrm{ppm}) 6.92(\mathrm{t}, J=7.2 \mathrm{~Hz}, 4 \mathrm{H}), 6.63(\mathrm{t}, J=7.5$ $\mathrm{Hz}, 2 \mathrm{H}), 3.88$ (brs, 2H), 3.40-3.35 (m, 2H), 2.87-2.78 (m, 4H), 2.15 (s, 6H), 2.04-2.00 (m, 2H), 1.96-1.85 (m, 2H); ${ }^{13} \mathrm{C}$ NMR (75 MHz, $\left.\mathrm{CDCl}_{3}\right): \delta(\mathrm{ppm}) 141.7,128.2,127.3,122.2$, $121.4,117.4,54.9,25.9,24.2,17.3$.

The enantiomeric excess was determined by HPLC on Chiralcel AD-H column (n-hexane : isopropanol $=98: 2$, flowing rate $=1.0 \mathrm{~mL} / \mathrm{min}, 20{ }^{\circ} \mathrm{C}$, UV detection at $\lambda=254$ $\mathrm{nm}), \mathrm{t}_{\mathrm{R} 1}=7.5 \min$ (major), $\mathrm{t}_{\mathrm{R} 2}=8.5 \min$ (minor).

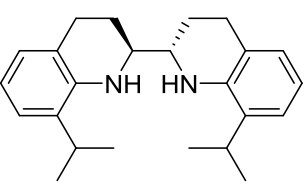

(2S,2'S)-2c (Known compound, see: Ma, W.; Zhang, J.; Xu, C.; Chen, F.; He, Y.-M.; Fan, Q.-H. Angew. Chem. Int. Ed. 2016, 55, 12891-12894). Following the similar synthetic procedure of $\left(2 S, 2^{\prime} S\right)-2 \mathbf{a}$ in $0.57 \mathrm{mmol}$ scale with $4.0 \mathrm{~mol} \%(R, R)-\mathbf{C 1 a}$ at $75{ }^{\circ} \mathrm{C}$ for $24 \mathrm{~h}$. The diastereoisomer was purified under slow flowing by silica gel column chromatography (eluent: petroleum ether/ethyl acetate = $300 / 1-100 / 1, v / v)$ to give the one of the pure isomer $(2 S, 2 ' S)-2 \mathbf{c}$ as yellow oil $(61 \mathrm{mg}, 30 \%$ yield, 99\% ee). ${ }^{1} \mathrm{H}$ NMR $\left(500 \mathrm{MHz}, \mathrm{CDCl}_{3}\right): \delta(\mathrm{ppm}) 7.02(\mathrm{~d}, J=8.0 \mathrm{~Hz}, 2 \mathrm{H}), 6.89$ (d, $J=7.5$ $\mathrm{Hz}, 2 \mathrm{H}), 6.70(\mathrm{t}, J=7.5 \mathrm{~Hz}, 2 \mathrm{H}), 4.49$ (brs, 2H), 3.38-3.37 (m, 2H), 2.92-2.83 (m, 6H), 2.03-2.01 (m, 2H), 1.89-1.86 (m, 2H), $1.26\left(\mathrm{dd}, J_{l}=15.0 \mathrm{~Hz}, J_{2}=7.0 \mathrm{~Hz}, 12 \mathrm{H}\right) ;{ }^{13} \mathrm{C} \mathrm{NMR}$ $\left(125 \mathrm{MHz}, \mathrm{CDCl}_{3}\right): \delta$ (ppm) 140.6, 132.7, 127.1, 123.1, 122.0, 117.6, 55.3, 27.4, 26.5, 24.5, 22.6, 22.4 .

The enantiomeric excess was determined by HPLC on Chiralcel OJ-H column (n-hexane : isopropanol $=98: 2$, flowing rate $=1.0 \mathrm{~mL} / \mathrm{min}, 20{ }^{\circ} \mathrm{C}$, UV detection at $\lambda=254$ $\mathrm{nm}), \mathrm{t}_{\mathrm{R} 1}=6.0 \min$ (major), $\mathrm{t}_{\mathrm{R} 2}=7.1 \mathrm{~min}$ (minor).

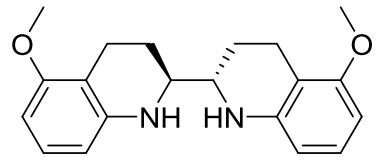

(2S,2'S)-2d (Known compound, see: Ma, W.; Zhang, J.; Xu, C.; Chen, F.; He, Y.-M.; Fan, Q.-H. Angew. Chem. Int. Ed. 2016, 55, 12891-12894). Following the similar synthetic procedure of $\left(2 S, 2^{\prime} S\right)-2 \mathbf{a}$ in $0.47 \mathrm{mmol}$ scale with $4.0 \mathrm{~mol} \%(R, R)-\mathbf{C 1 a}$ at $50{ }^{\circ} \mathrm{C}$ for $48 \mathrm{~h}$. The 
diastereoisomer was purified under slow flowing by silica gel column chromatography (eluent: petroleum ether/ethyl acetate $=100 / 1-50 / 1, v / v)$ to give the one of the pure isomer $\left(2 S, 2^{\prime} S\right)-\mathbf{2 d}$ as pale yellow oil (121 mg, 79\% yield, 99\% ee). ${ }^{1} \mathrm{H} \mathrm{NMR}\left(500 \mathrm{MHz}, \mathrm{CDCl}_{3}\right): \delta(\mathrm{ppm}) 6.96(\mathrm{t}$, $J=8.0 \mathrm{~Hz}, 2 \mathrm{H}), 6.25(\mathrm{~d}, J=8.5 \mathrm{~Hz}, 4 \mathrm{H}), 3.80(\mathrm{~s}, 6 \mathrm{H}), 3.26-3.23(\mathrm{~m}, 2 \mathrm{H}), 2.78-2.72(\mathrm{~m}, 2 \mathrm{H})$, 2.66-2.60 (m, 2H), 2.01-1.96 (m, 2H), 1.85-1.78 (m, 2H); $\left.{ }^{13} \mathrm{C} \mathrm{NMR} \mathrm{(125} \mathrm{MHz,} \mathrm{CDCl}_{3}\right): \delta$ (ppm) 157.9, 145.0, 127.0, 110.1, 108.3, 99.7, 55.4, 54.1, 23.9, 19.6.

The enantiomeric excess was determined by HPLC on Chiralcel AD-H column (n-hexane : isopropanol $=90: 10$, flowing rate $=1.0 \mathrm{~mL} / \mathrm{min}, 20{ }^{\circ} \mathrm{C}$, $\mathrm{UV}$ detection at $\lambda=254$ $\mathrm{nm}), \mathrm{t}_{\mathrm{R} 1}=14.1 \mathrm{~min}$ (major), $\mathrm{t}_{\mathrm{R} 2}=17.2 \mathrm{~min}$ (minor).

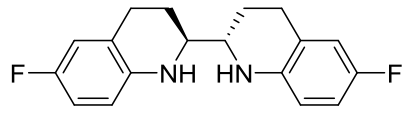

(2S,2'S)-2e (Known compound, see: Ma, W.; Zhang, J.; Xu, C.;

Chen, F.; He, Y.-M.; Fan, Q.-H. Angew. Chem. Int. Ed. 2016, 55, 12891-12894). Following the similar synthetic procedure of $(2 S, 2 ' S)-2 \mathbf{a}$ in $0.51 \mathrm{mmol}$ scale with $4.0 \mathrm{~mol} \%(R, R)-\mathbf{C 1 a}$ at $50{ }^{\circ} \mathrm{C}$ for $48 \mathrm{~h}$. The diastereoisomer was purified under slow flowing by silica gel column chromatography (eluent: petroleum ether/ethyl acetate = $100 / 1-20 / 1, v / v)$ to give the one of the pure isomer $(2 S, 2$ 'S)-2e as pale yellow oil $(100 \mathrm{mg}, 65 \%$ yield, 99\% ee). ${ }^{1} \mathrm{H}$ NMR (400 MHz, $\left.\mathrm{CDCl}_{3}\right): \delta(\mathrm{ppm}) 6.62(\mathrm{~d}, J=8.4 \mathrm{~Hz}, 4 \mathrm{H}), 6.45-6.41(\mathrm{~m}$, 2H), $3.83(\mathrm{~s}, 2 \mathrm{H}), 3.15-3.14(\mathrm{~m}, 2 \mathrm{H}), 2.69-2.63(\mathrm{~m}, 4 \mathrm{H}), 1.92-1.83(\mathrm{~m}, 2 \mathrm{H}), 1.75-1.69(\mathrm{~m}$, $2 \mathrm{H}) ;{ }^{13} \mathrm{C}$ NMR $\left(100 \mathrm{MHz}, \mathrm{CDCl}_{3}\right): \delta$ (ppm) 157.4, 154.3, 140.3, 123.2, 123.1, 115.9, 115.8, $115.6,115.3,113.7,113.4,55.0,26.1,24.2$.

The enantiomeric excess was determined by HPLC on Chiralcel AD-H column (n-hexane : isopropanol $=80: 20$, flowing rate $=1.0 \mathrm{~mL} / \mathrm{min}, 20{ }^{\circ} \mathrm{C}$, UV detection at $\lambda=254$ $\mathrm{nm}), \mathrm{t}_{\mathrm{R} 1}=9.5 \min$ (minor), $\mathrm{t}_{\mathrm{R} 2}=12.1 \min$ (major).

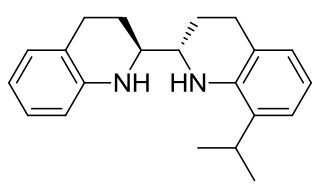

(2S,2'S)-2f (New compound): Following the similar synthetic procedure of $(2 S, 2 ' S)$-2a in $1.5 \mathrm{mmol}$ scale. The diastereoisomer was purified under slow flowing by silica gel column chromatography (eluent: petroleum ether/ethyl acetate $=300 / 1-100 / 1, v / v)$ to give the one of the pure isomer $\left(2 S, 2^{\prime} S\right)$-2f as pale yellow oil (300 mg, 65\% yield, 99\% ee). $[\alpha]_{\mathrm{D}}{ }^{20}=+64\left(c=0.2, \mathrm{CH}_{2} \mathrm{Cl}_{2}\right) ;{ }^{1} \mathrm{H} \mathrm{NMR}(500 \mathrm{MHz}$, 
$\left.\mathrm{CDCl}_{3}\right): \delta(\mathrm{ppm}) 7.02(\mathrm{t}, J=8.0 \mathrm{~Hz}, 3 \mathrm{H}), 6.90(\mathrm{~d}, J=7.5 \mathrm{~Hz}, 1 \mathrm{H}), 6.72-6.66(\mathrm{~m}, 2 \mathrm{H}), 6.58(\mathrm{~d}$, $J=7.5 \mathrm{~Hz}, 1 \mathrm{H}), 4.13$ (brs, 2H), 3.39-3.35 (m, 2H), 2.93-2.78 (m, 5H), 2.07-1.96 (m, 2H), $1.93-1.84(\mathrm{~m}, 2 \mathrm{H}), 1.28\left(\mathrm{dd}, J_{1}=14.3 \mathrm{~Hz}, J_{2}=6.8 \mathrm{~Hz}, 6 \mathrm{H}\right) ;{ }^{13} \mathrm{C} \mathrm{NMR}\left(125 \mathrm{MHz}, \mathrm{CDCl}_{3}\right): \delta$ (ppm) 144.2, 140.7, 132.4, 129.4, 127.1, 127.0, 123.1, 121.8, 121.7, 117.7, 117.4, 115.0, 55.2, 54.9, 27.4, 26.4, 26.0, 24.6, 24.1, 22.5, 22.4. HRMS-ESI exact mass calcd. for $\mathrm{C}_{21} \mathrm{H}_{27} \mathrm{~N}_{2}$ $\left([\mathrm{M}+\mathrm{H}]^{+}\right)$requires $\mathrm{m} / \mathrm{z}$ 307.21688, found $\mathrm{m} / \mathrm{z} 307.21662$.

The enantiomeric excess was determined by HPLC on Chiralcel AD-H column (n-hexane : isopropanol $=90: 10$, flowing rate $=1.0 \mathrm{~mL} / \mathrm{min}, 20{ }^{\circ} \mathrm{C}$, UV detection at $\lambda=254$ $\mathrm{nm}), \mathrm{t}_{\mathrm{R} 1}=5.3 \min$ (minor), $\mathrm{t}_{\mathrm{R} 2}=6.9 \min$ (major).

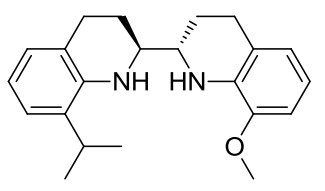

(2S,2'S)-2g (New compound): Following the similar synthetic procedure of $(2 S, 2 ' S)-2 \mathbf{a}$ in $3.6 \mathrm{mmol}$ scale with $4.0 \mathrm{~mol} \%(R, R)-\mathbf{C 1 a}$ at $50{ }^{\circ} \mathrm{C}$. The diastereoisomer was purified under slow flowing by silica gel column chromatography (eluent: petroleum ether/ethyl acetate $=200 / 1-50 / 1, v / v$ ) to give the one of the pure isomer $\left(2 S, 2^{\prime} S\right)-2 \mathrm{~g}$ as colorless oil $\left(944 \mathrm{mg}, 77 \%\right.$ yield, $99 \%$ ee). $[\alpha]_{\mathrm{D}}{ }^{20}=+84(c=$ 1.0, $\left.\mathrm{CH}_{2} \mathrm{Cl}_{2}\right) ;{ }^{1} \mathrm{H} \mathrm{NMR}\left(500 \mathrm{MHz}, \mathrm{CDCl}_{3}\right): \delta(\mathrm{ppm}) 7.01(\mathrm{~d}, J=7.5 \mathrm{~Hz}, 1 \mathrm{H}), 6.88(\mathrm{~d}, J=7.5$ $\mathrm{Hz}, 1 \mathrm{H}), 6.69-6.59(\mathrm{~m}, 4 \mathrm{H}), 4.35$ (brs, 2H), 3.82 (s, 3H), 3.40-3.32 (m, 2H), 2.91-2.78 (m, 5H), 2.08-1.97 (m, 2H), 1.93-1.86 (m, 2H), $1.29(\mathrm{~d}, J=6.5 \mathrm{~Hz}, 3 \mathrm{H}), 1.26(\mathrm{~d}, J=6.5 \mathrm{~Hz}, 3 \mathrm{H})$; ${ }^{13} \mathrm{C} \mathrm{NMR}\left(125 \mathrm{MHz}, \mathrm{CDCl}_{3}\right): \delta$ (ppm) 146.6, 140.8, 133.9, 132.2, 127.1, 123.1, 121.6, 121.5, $117.1,116.4,107.5,55.5,55.0,54.2,27.4,27.1,26.2,25.7,24.5,23.9,22.4,22.3$. HRMS-ESI exact mass calcd. for $\mathrm{C}_{22} \mathrm{H}_{29} \mathrm{ON}_{2}\left([\mathrm{M}+\mathrm{H}]^{+}\right)$requires $\mathrm{m} / \mathrm{z} 337.22744$, found $\mathrm{m} / \mathrm{z} 337.22723$.

The enantiomeric excess was determined by HPLC on the connection of two Chiralcel AD-H column (n-hexane : isopropanol $=99: 1$, flowing rate $=0.5 \mathrm{~mL} / \mathrm{min}, 20{ }^{\circ} \mathrm{C}, \mathrm{UV}$ detection at $\lambda=254 \mathrm{~nm}$ ), $\mathrm{t}_{\mathrm{R} 1}=22.7 \min$ (major), $\mathrm{t}_{\mathrm{R} 2}=24.1 \mathrm{~min}$ (minor).

The chiral hydrogenated products $\mathbf{5}$ could be synthesized according to our reported procedures. ${ }^{[4]} 99 \%$ ee of products were given after recrystallization for one or two times. Among them, $(S)-\mathbf{5 a}$ and $(S)-\mathbf{5 c}$ are the known compounds. 


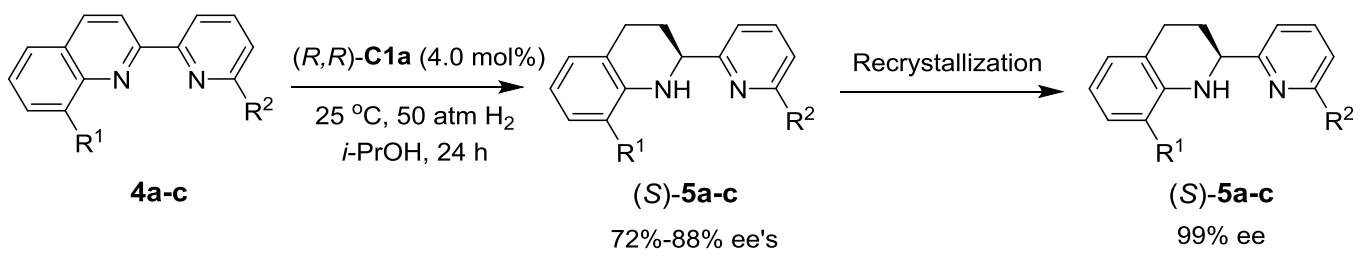

Typical procedure: In a $50 \mathrm{~mL}$ glass-lined stainless steel reactor with a magnetic stirring bar was charged with Ru-catalyst $(R, R)$-C1a $(120 \mathrm{mg}, 0.16 \mathrm{mmol}), 4 \mathbf{a}(889 \mathrm{mg}, 4.0 \mathrm{mmol})$ and $i$-PrOH $(10 \mathrm{~mL})$. The autoclave was closed, and the final pressure of the hydrogen gas was adjusted to $50 \mathrm{~atm}$ after purging the autoclave with hydrogen gas several times. The reaction mixture was stirred at $25{ }^{\circ} \mathrm{C}$ for $24 \mathrm{~h}$. Then the hydrogen gas was carefully released and the conversion was determined by ${ }^{1} \mathrm{H}$ NMR spectroscopy after the solvent was evaporated under reduced pressure. After concentration, the residue was purified by silica gel column chromatography (eluent: petroleum ether/ethyl acetate $=50 / 1-20 / 1, v / v$ ) to give the pure product (S)-5a (896 mg, 99\% yield, 87\% ee). It was further recrystallized from ethyl acetate to give $(S)-5 a$ (White solid, $715 \mathrm{mg}$, 79\% yield, 99\% ee).

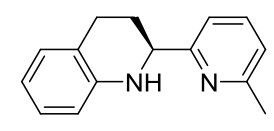

(S)-5a (Known compound, see: Liu, Y.; Chen, F.; He, Y.-M.; Li, C.; Fan, Q.-H. Org. Biomol. Chem. 2019, 17, 5099-5105). White solid, 715 mg, 79\% yield, 99\% ee, $[\alpha]_{\mathrm{D}}{ }^{20}=-110\left(c=1.0, \mathrm{CHCl}_{3}\right) .{ }^{1} \mathrm{H} \mathrm{NMR}\left(300 \mathrm{MHz}, \mathrm{CDCl}_{3}\right): \delta(\mathrm{ppm}) 7.55(\mathrm{t}, J$ $=7.8 \mathrm{~Hz}, 1 \mathrm{H}), 7.22(\mathrm{~d}, J=7.8 \mathrm{~Hz}, 1 \mathrm{H}), 7.05-6.97(\mathrm{~m}, 3 \mathrm{H}), 6.67-6.60(\mathrm{~m}, 2 \mathrm{H}), 4.56-4.52(\mathrm{~m}$, $2 \mathrm{H}$, containing NH ), 2.97-2.86 (m, 1H), 2.73-2.65 (m, 1H), $2.56(\mathrm{~s}, 3 \mathrm{H}), 2.30-2.21(\mathrm{~m}, 1 \mathrm{H})$, 2.05-1.93 (m, 1H); ${ }^{13} \mathrm{C}$ NMR (75 MHz, $\left.\mathrm{CDCl}_{3}\right): \delta(\mathrm{ppm}) 162.6,157.9,144.4,137.1,129.3$, 127.0, 121.8, 121.2, 117.5, 117.3, 114.5, 57.0, 28.9, 26.2, 24.6.

The enantiomeric excess was determined by HPLC on Chiralcel OB-H column (n-hexane : isopropanol $=95: 5$, flowing rate $=1.0 \mathrm{~mL} / \mathrm{min}, 25^{\circ} \mathrm{C}$, $\mathrm{UV}$ detection at $\lambda=254$ $\mathrm{nm}), \mathrm{t}_{\mathrm{R} 1}=10.5 \min ($ minor $), \mathrm{t}_{\mathrm{R} 2}=11.9 \min$ (major).

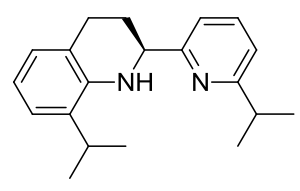

(S)-5b (New compound): Following the similar synthetic procedure of (S)-5a in $1.01 \mathrm{mmol}$ scale. The crude product was purified by silica gel column chromatography (eluent: petroleum ether / ethyl acetate $=$ 
50/1-20/1, v/v) to give the pure product $(S)$-5b (Colorless oil, $214 \mathrm{mg}, 72 \%$ yield, $88 \%$ ee). To further improve the enantiomeric excess to $99 \%$ ee, $(S)-5 \mathbf{b}(214 \mathrm{mg}, 0.73 \mathrm{mmol})$ was dissolved in $10 \mathrm{~mL} \mathrm{CH}_{2} \mathrm{Cl}_{2}$ at $25{ }^{\circ} \mathrm{C}$, trifluoromethanesulfonate $(109 \mathrm{mg}, 0.73 \mathrm{mmol})$ was added slowly, then it was stirred at $25{ }^{\circ} \mathrm{C}$ for $30 \mathrm{~min}$. The solvent was evaporated under reduced pressure to give the yellow solid (323 mg, 100\% yield). The yellow solid was recrystallized from ethyl acetate and petroleum ether. It was filtered and the solvent was washed with saturated $\mathrm{NaHCO}_{3}$, the organic layer was dried over $\mathrm{Na}_{2} \mathrm{SO}_{4}$, filtered, and evaporated in vacuo to give the pure $(S)-5 \mathbf{b}$ as colorless oil (140 mg, 47\% yield, $99 \%$ ee), $[\alpha]_{\mathrm{D}}{ }^{20}=-184\left(c=0.88, \mathrm{CHCl}_{3}\right) .{ }^{1} \mathrm{H}$ NMR $\left(300 \mathrm{MHz}, \mathrm{CDCl}_{3}\right): \delta(\mathrm{ppm}) 7.61(\mathrm{t}, J=7.7 \mathrm{~Hz}$, $1 \mathrm{H}), 7.21(\mathrm{~d}, J=7.8 \mathrm{~Hz}, 1 \mathrm{H}), 7.06\left(\mathrm{dd}, J_{l}=10.4 \mathrm{~Hz}, J_{2}=8.0 \mathrm{~Hz}, 2 \mathrm{H}\right), 6.89(\mathrm{~d}, J=7.2 \mathrm{~Hz}$, 1H), 6.67 (t, $J=7.5 \mathrm{~Hz}, 1 \mathrm{H}), 5.09$ (brs, $1 \mathrm{H}), 4.59$ (d, $J=7.8 \mathrm{~Hz}, 1 \mathrm{H}), 3.13-2.92(\mathrm{~m}, 3 \mathrm{H}), 2.78$ $\left(\mathrm{dt}, J_{1}=10.4 \mathrm{~Hz}, J_{2}=4.5 \mathrm{~Hz}, 1 \mathrm{H}\right), 2.34-2.26(\mathrm{~m}, 1 \mathrm{H}) 1.93-1.81(\mathrm{~m}, 1 \mathrm{H}), 1.32\left(\mathrm{dd}, J_{l}=6.8 \mathrm{~Hz}\right.$, $\left.J_{2}=2.6 \mathrm{~Hz}, 12 \mathrm{H}\right) ;{ }^{13} \mathrm{C} \mathrm{NMR}\left(75 \mathrm{MHz}, \mathrm{CDCl}_{3}\right): \delta(\mathrm{ppm}) 166.4,161.7,141.1,137.2,132.1$, $127.0,123.1,121.0,119.0,117.9,116.7,56.7,36.3,28.9,27.4,22.7,22.7,22.7,22.4$. HRMS-ESI exact mass calcd. for $\mathrm{C}_{20} \mathrm{H}_{27} \mathrm{~N}_{2}\left([\mathrm{M}+\mathrm{H}]^{+}\right)$requires $\mathrm{m} / \mathrm{z} 295.21688$, found $\mathrm{m} / \mathrm{z}$ 295.21648.

The enantiomeric excess was determined by HPLC on the connection of two Chiralcel AD-H column. $\left(n\right.$-hexane $:$ isopropanol $=99.5: 0.5$, flowing rate $=0.5 \mathrm{~mL} / \mathrm{min}, 25{ }^{\circ} \mathrm{C}, \mathrm{UV}$ detection at $\lambda=254 \mathrm{~nm}$ ), $\mathrm{t}_{\mathrm{R} 1}=18.0 \mathrm{~min}$ (major), $\mathrm{t}_{\mathrm{R} 2}=19.4 \mathrm{~min} \quad$ (minor).

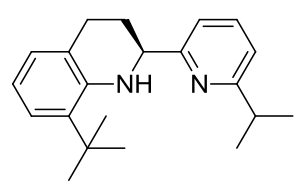

(S)-5c (Known compound, see: Liu, Y.; Chen, F.; He, Y.-M.; Li, C.; Fan, Q.-H. Org. Biomol. Chem. 2019, 17, 5099-5105). Following the similar synthetic procedure of $(S)-\mathbf{5 a}$ in $1.95 \mathrm{mmol}$ scale. The crude product was purified by silica gel column chromatography (eluent: petroleum ether/ethyl acetate = 50/1-20/1, v/v) to give the pure product $(S)-5 \mathbf{c}$ (Colorless oil, $522 \mathrm{mg}, 87 \%$ yield, $72 \%$ ee). To further improve the enantiomeric excess to $99 \%$ ee, $(S)-5 c(522 \mathrm{mg}, 1.69 \mathrm{mmol})$ was dissolved in $10 \mathrm{~mL} \mathrm{CH}_{2} \mathrm{Cl}_{2}$ at $25{ }^{\circ} \mathrm{C}$, trifluoromethanesulfonate (254 mg, $1.69 \mathrm{mmol}$ ) was added slowly, then it was stirred at $25{ }^{\circ} \mathrm{C}$ for $30 \mathrm{~min}$. The solvent was evaporated under reduced pressure to give the yellow solid (776 mg, 100\% yield). The yellow solid was 
recrystallized from ethyl acetate and petroleum ether for two times. It was filtered and the yellow solid was dissolved in $\mathrm{CH}_{2} \mathrm{Cl}_{2}$, washed with saturated $\mathrm{NaHCO}_{3}$, dried over $\mathrm{Na}_{2} \mathrm{SO}_{4}$, filtered, and evaporated in vacuo to give the pure $(S)-5 \mathbf{c}$ as colorless oil (138 $\mathrm{mg}, 23 \%$ yield, $99 \%$ ee $),[\alpha]_{\mathrm{D}}{ }^{20}=-194\left(c=0.65, \mathrm{CHCl}_{3}\right) .{ }^{1} \mathrm{H} \mathrm{NMR}\left(400 \mathrm{MHz}, \mathrm{CDCl}_{3}\right): \delta(\mathrm{ppm}) 7.60(\mathrm{t}, J=$ $7.8 \mathrm{~Hz}, 1 \mathrm{H}), 7.19(\mathrm{~d}, J=7.6 \mathrm{~Hz}, 1 \mathrm{H}), 7.13(\mathrm{~d}, J=7.2 \mathrm{~Hz}, 1 \mathrm{H}), 7.06(\mathrm{~d}, J=7.6 \mathrm{~Hz}, 1 \mathrm{H}), 6.91$ $(\mathrm{d}, J=7.2 \mathrm{~Hz}, 1 \mathrm{H}), 6.60(\mathrm{t}, J=7.6 \mathrm{~Hz}, 1 \mathrm{H}), 5.62$ (brs, $1 \mathrm{H}), 4.57(\mathrm{~d}, J=8.8 \mathrm{~Hz}, 1 \mathrm{H})$, 3.11-3.03 (m, 2H), $2.84\left(\mathrm{dt}, J_{l}=16.4 \mathrm{~Hz}, J_{2}=4.2 \mathrm{~Hz}, 1 \mathrm{H}\right), 2.37-2.31(\mathrm{~m}, 1 \mathrm{H}), 1.91-1.82(\mathrm{~m}$, $1 \mathrm{H}), 1.50(\mathrm{~s}, 9 \mathrm{H}), 1.32\left(\mathrm{dd}, J_{l}=7.0 \mathrm{~Hz}, J_{2}=1.8 \mathrm{~Hz}, 6 \mathrm{H}\right) ;{ }^{13} \mathrm{C} \mathrm{NMR}\left(75 \mathrm{MHz}, \mathrm{CDCl}_{3}\right): \delta(\mathrm{ppm})$ 166.4, 161.1 142.6, 137.1, 132.9, 127.7, 124.4, 121.3, 119.0, 117.7, 116.1, 56.4, 36.5, 34.4, 30.0, 28.4, 28.1, 22.8, 22.7. HRMS-ESI exact mass calcd. for $\mathrm{C}_{21} \mathrm{H}_{29} \mathrm{~N}_{2}\left([\mathrm{M}+\mathrm{H}]^{+}\right)$requires $\mathrm{m} / \mathrm{z} 309.23253$, found $\mathrm{m} / \mathrm{z} 309.23213$.

The enantiomeric excess was determined by HPLC on the connection of two Chiralcel AD-H column. (n-hexane : isopropanol $=99.5: 0.5$, flowing rate $=0.5 \mathrm{~mL} / \mathrm{min}, 25{ }^{\circ} \mathrm{C}, \mathrm{UV}$ detection at $\lambda=254 \mathrm{~nm}$ ), $\mathrm{t}_{\mathrm{R} 1}=18.2 \min$ (major), $\mathrm{t}_{\mathrm{R} 2}=19.4 \min$ (minor). 


\subsection{General procedure for $\mathrm{Hydrogenation} \mathrm{of}(S)-5$ with $\mathrm{PtO}_{2}$.}

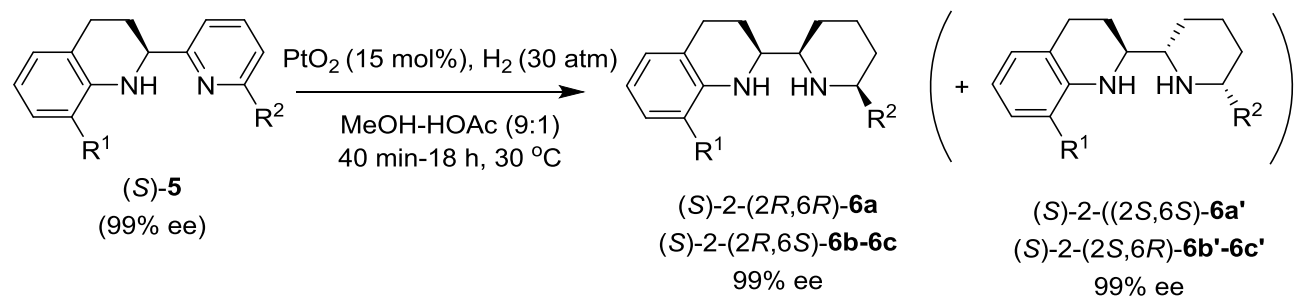

Typical procedure: A $50 \mathrm{~mL}$ glass-lined stainless-steel reactor equipped with a magnetic stirrer bar was charged with $\mathrm{PtO}_{2}(0.32 \mathrm{mmol}, 72 \mathrm{mg})$ and $(S)-5 \mathbf{a}(474 \mathrm{mg}, 2.1 \mathrm{mmol})$ in $\mathrm{MeOH}(4.5 \mathrm{~mL})$ containing HOAc $(0.5 \mathrm{~mL})$. The autoclave was closed, and the final pressure of the hydrogen gas was adjusted to 30 atm after purging the autoclave with hydrogen gas several times. The reaction mixture was stirred at $30{ }^{\circ} \mathrm{C}$ for $40 \mathrm{~min}$. Then the hydrogen gas was carefully released and the solvent was evaporated. The residue was dissolved in $\mathrm{CH}_{2} \mathrm{Cl}_{2}$, washed with sat. aq. $\mathrm{Na}_{2} \mathrm{CO}_{3}$ solution and brine, and dried over $\mathrm{Na}_{2} \mathrm{SO}_{4}$. The diastereoisomer was purified under slow flowing by silica gel column chromatography (eluent: petroleum ether/ethyl acetate $\left./ \mathrm{Et}_{3} \mathrm{~N}=150 / 10 / 1-70 / 10 / 1, v / v / v\right)$ to give the one of the pure isomer (S)-2-(2R,6R)-6a (colorless oil, $298 \mathrm{mg}, 61 \%$ yield, 99\% ee).

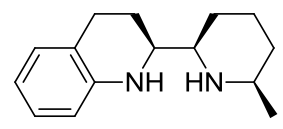

(S)-2-(2R,6R)-6a (New compound): Colorless oil, $298 \mathrm{mg}, 61 \%$ yield, 99\% ee. $[\alpha]_{\mathrm{D}}{ }^{20}=+34.4\left(c=0.55, \mathrm{CHCl}_{3}\right) ;{ }^{1} \mathrm{H} \mathrm{NMR}\left(300 \mathrm{MHz}, \mathrm{CDCl}_{3}\right): \delta(\mathrm{ppm})$ $6.97(\mathrm{t}, J=8.1 \mathrm{~Hz}, 2 \mathrm{H}), 6.60(\mathrm{t}, J=7.4 \mathrm{~Hz}, 1 \mathrm{H}), 6.53(\mathrm{~d}, J=7.8 \mathrm{~Hz}, 1 \mathrm{H}), 4.18(\mathrm{~s}, 1 \mathrm{H})$, 3.26-3.21 (m, 1H), 2.91-2.68 (m, 4H), 1.89-1.75 (m, 3H), 1.64-1.46 (m, 3H, containing NH), 1.44-1.25 (m, 2H), 1.15-1.02 (m, 1H), $1.10(\mathrm{~d}, J=6.3 \mathrm{~Hz}, 3 \mathrm{H}) ;{ }^{13} \mathrm{C}$ NMR $\left(75 \mathrm{MHz}, \mathrm{CDCl}_{3}\right)$ : $\delta(\mathrm{ppm}) 145.1,129.2,126.8,121.4,116.7,114.1,60.9,56.3,52.9,34.3,26.9,26.2,24.7,24.2$, 23.2. HRMS (ESI) m/z: $[\mathrm{M}+\mathrm{H}]^{+}$Calcd for $\mathrm{C}_{15} \mathrm{H}_{23} \mathrm{~N}_{2}$ 231.18558; Found 231.18513.

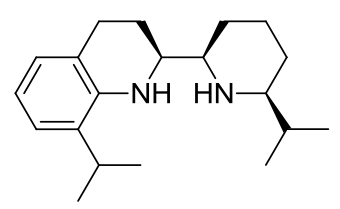

(S)-2-(2R,6S)-6b (New compound): Following the similar synthetic procedure of $(S)-2-(2 R, 6 R)-6 \mathbf{a}$ in $1.24 \mathrm{mmol}$ scale for $18 \mathrm{~h}$. The diastereoisomer was purified under slow flowing by silica gel column chromatography (eluent: petroleum ether / ethyl acetate $/ \mathrm{Et}_{3} \mathrm{~N}=300 / 10 / 1-100 / 10 / 1, v / v / v$ ) to give the one of the pure isomer $(S)-2-(2 R, 6 S)-6 \mathbf{b}$ as colorless oil (302 mg, 81\% yield, $99 \%$ ee). 
$[\alpha]_{\mathrm{D}}{ }^{20}=+38.8\left(c=0.8, \mathrm{CH}_{2} \mathrm{Cl}_{2}\right) ;{ }^{1} \mathrm{H} \mathrm{NMR}\left(300 \mathrm{MHz}, \mathrm{CDCl}_{3}\right): \delta(\mathrm{ppm}) 6.97(\mathrm{~d}, J=7.5 \mathrm{~Hz}$, $1 \mathrm{H}), 6.85(\mathrm{~d}, J=7.2 \mathrm{~Hz}, 1 \mathrm{H}), 6.60(\mathrm{t}, J=7.5 \mathrm{~Hz}, 1 \mathrm{H}), 4.48(\mathrm{~s}, 1 \mathrm{H}), 3.30-3.26(\mathrm{~m}, 1 \mathrm{H})$, 2.96-2.74 (m, 4H), 2.41-2.36 (m, 1H), 1.88-1.72 (m, 3H), 1.64-1.52 (m, 3H), 1.40-1.26 (m, $3 \mathrm{H}), 1.27\left(\mathrm{dd}, J_{1}=6.9 \mathrm{~Hz}, J_{2}=1.2 \mathrm{~Hz}, 6 \mathrm{H}\right), 1.15-1.03(\mathrm{~m}, 1 \mathrm{H}), 0.94(\mathrm{~d}, J=6.6 \mathrm{~Hz}, 6 \mathrm{H}) ;{ }^{13} \mathrm{C}$ NMR (75 MHz, $\left.\mathrm{CDCl}_{3}\right): \delta(\mathrm{ppm}) 142.0,131.0,126.9,122.9,121.0,116.1,62.7,60.5,56.6$, 33.6, 28.7, 27.7, 27.5, 26.2, 24.7, 24.1, 22.3, 22.1, 18.8, 18.7. HRMS (ESI) m/z: $[\mathrm{M}+\mathrm{H}]^{+}$ Calcd for $\mathrm{C}_{20} \mathrm{H}_{33} \mathrm{~N}_{2}$ 301.26383; Found 301.26336.

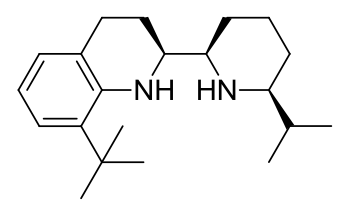

$(S)-2-(2 R, 6 S)-6 \mathbf{c}$ (New compound): Following the similar synthetic procedure of $(S)-2-(2 R, 6 R)-6 \mathbf{a}$ in $0.42 \mathrm{mmol}$ scale for $18 \mathrm{~h}$. The diastereoisomer was purified under slow flowing by silica gel column chromatography (eluent: petroleum ether / ethyl acetate $/ \mathrm{Et}_{3} \mathrm{~N}=300 / 10 / 1-100 / 10 / 1, v / v / v$ ) to give the one of the pure isomer $(S)-2-(2 R, 6 S)-6 \mathbf{c}$ as colorless oil (106 mg, $80 \%$ yield, $99 \%$ ee). $[\alpha]_{\mathrm{D}}{ }^{20}=+41.5\left(c=1.0, \mathrm{CH}_{2} \mathrm{Cl}_{2}\right) ;{ }^{1} \mathrm{H}$ NMR $\left(300 \mathrm{MHz}, \mathrm{CDCl}_{3}\right): \delta(\mathrm{ppm}) 7.07(\mathrm{~d}, J=7.8 \mathrm{~Hz}$, $1 \mathrm{H}), 6.85(\mathrm{~d}, J=7.2 \mathrm{~Hz}, 1 \mathrm{H}), 6.53(\mathrm{t}, J=7.7 \mathrm{~Hz}, 1 \mathrm{H}), 4.94(\mathrm{~s}, 1 \mathrm{H}), 3.36-3.30(\mathrm{~m}, 1 \mathrm{H})$, 3.00-2.89 (m, 1H), 2.83-2.75 (m, 2H), 2.48-2.42 (m, 1H), 1.89-1.75 (m, 3H), 1.62-1.54 (m, $3 \mathrm{H}), 1.43-1.22(\mathrm{~m}, 3 \mathrm{H}), 1.43(\mathrm{~s}, 9 \mathrm{H}), 1.16-1.07(\mathrm{~m}, 1 \mathrm{H}), 0.94\left(\mathrm{dd}, J_{1}=6.9 \mathrm{~Hz}, J_{2}=3.6 \mathrm{~Hz}\right.$, $6 \mathrm{H}) ;{ }^{13} \mathrm{C} \mathrm{NMR}\left(75 \mathrm{MHz}, \mathrm{CDCl}_{3}\right): \delta(\mathrm{ppm}) 143.5,132.0,127.6,124.3,121.3,115.4,62.6,60.3$, 56.8, 34.2, 33.7, 29.9, 28.5, 28.4, 25.5, 24.6, 24.1, 18.7, 18.6. HRMS (ESI) m/z: $[\mathrm{M}+\mathrm{H}]^{+}$ Calcd for $\mathrm{C}_{21} \mathrm{H}_{35} \mathrm{~N}_{2}$ 315.27948; Found 315.27896.

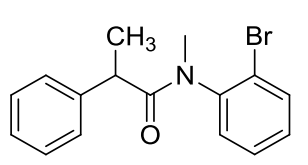

14 (Known compound, see: Lee, S.; Hartwig, J. F. J. Org. Chem. 2001, 66, 3402). ${ }^{1} \mathrm{H}$ NMR (300 MHz, $\left.\mathrm{CDCl}_{3}\right): \delta(\mathrm{ppm}) \quad 7.72-7.69(\mathrm{~m}, 0.7 \mathrm{H})$, 7.59-7.56 (m, 0.3H), 7.46-7.34 (m, 0.8H), 7.29-7.13 (m, 5.4H), 7.02-6.94 $(\mathrm{m}, 2.2 \mathrm{H}), 6.72-6.69(\mathrm{~m}, 0.8 \mathrm{H}), 3.52(\mathrm{q}, J=6.9 \mathrm{~Hz}, 0.3 \mathrm{H}), 3.35(\mathrm{q}, J=6.9 \mathrm{~Hz}, 0.8 \mathrm{H}), 3.19(\mathrm{~s}$, $0.88 \mathrm{H}), 3.17(\mathrm{~s}, 2.5 \mathrm{H}), 1.43(\mathrm{~d}, J=6.9 \mathrm{~Hz}, 3 \mathrm{H}) ;{ }^{13} \mathrm{C} \mathrm{NMR}\left(75 \mathrm{MHz}, \mathrm{CDCl}_{3}\right): \delta 173.9,142.4$, $141.8,140.7,134.2,133.7,131.0,130.2,129.8,129.8,128.8,128.6,128.5,128.3,128.2$, $127.6,126.9,126.8,123.8,44.2,43.4,36.3,20.8,20.2$. 


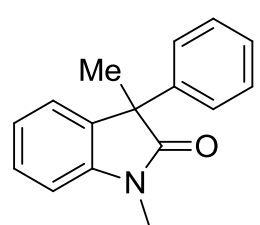

15 (Known compound, see: Lee, S.; Hartwig, J. F. J. Org. Chem. 2001, 66, 3402). ${ }^{1} \mathrm{H}$ NMR (300 MHz, $\left.\mathrm{CDCl}_{3}\right): \delta 7.34-7.11(\mathrm{~m}, 7 \mathrm{H}), 7.07(\mathrm{~d}, J=7.5 \mathrm{~Hz}$, $1 \mathrm{H}), 6.91(\mathrm{~d}, J=7.8 \mathrm{~Hz}, 1 \mathrm{H}), 3.23(\mathrm{~s}, 3 \mathrm{H}), 1.79(\mathrm{~s}, 3 \mathrm{H}) .{ }^{13} \mathrm{C}$ NMR $(125$ $\left.\mathrm{MHz}, \mathrm{CDCl}_{3}\right): \delta(\mathrm{ppm}) 179.5,143.3,140.9,134.9,128.6,128.2,127.3,126.7,124.3,122.9$, 52.2, 26.6, 23.9. 


\section{NMR and HPLC spectra of compounds}

Figure S9. ${ }^{1} \mathrm{H}$ NMR and ${ }^{13} \mathrm{C}$ NMR spectra of $\mathbf{1 f}\left(\mathrm{CDCl}_{3}\right)$

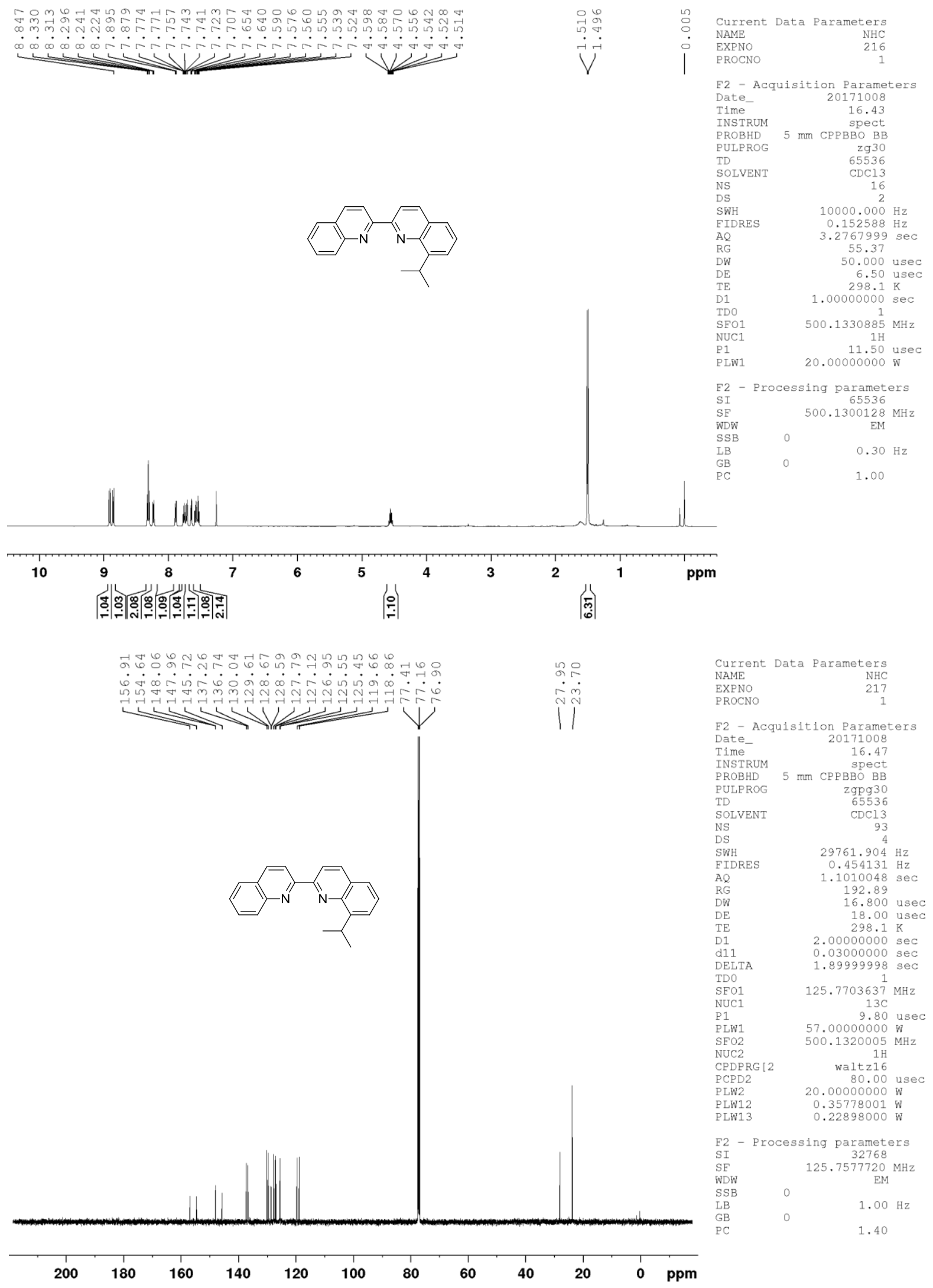


Figure S10. ${ }^{1} \mathrm{H}$ NMR and ${ }^{13} \mathrm{C}$ NMR spectra of $\mathbf{1 g}\left(\mathrm{CDCl}_{3}\right)$

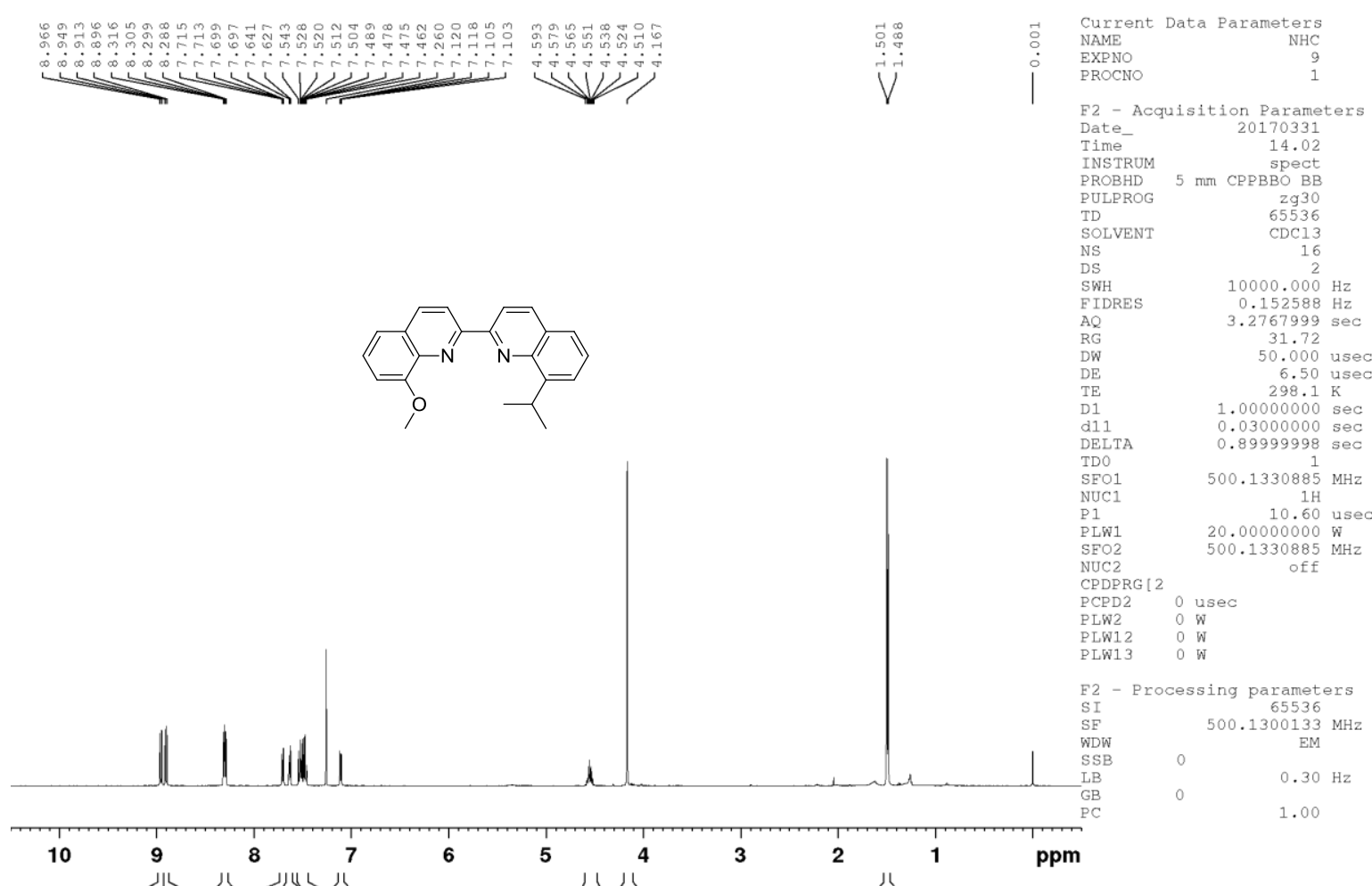

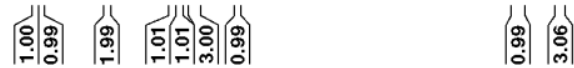

荌

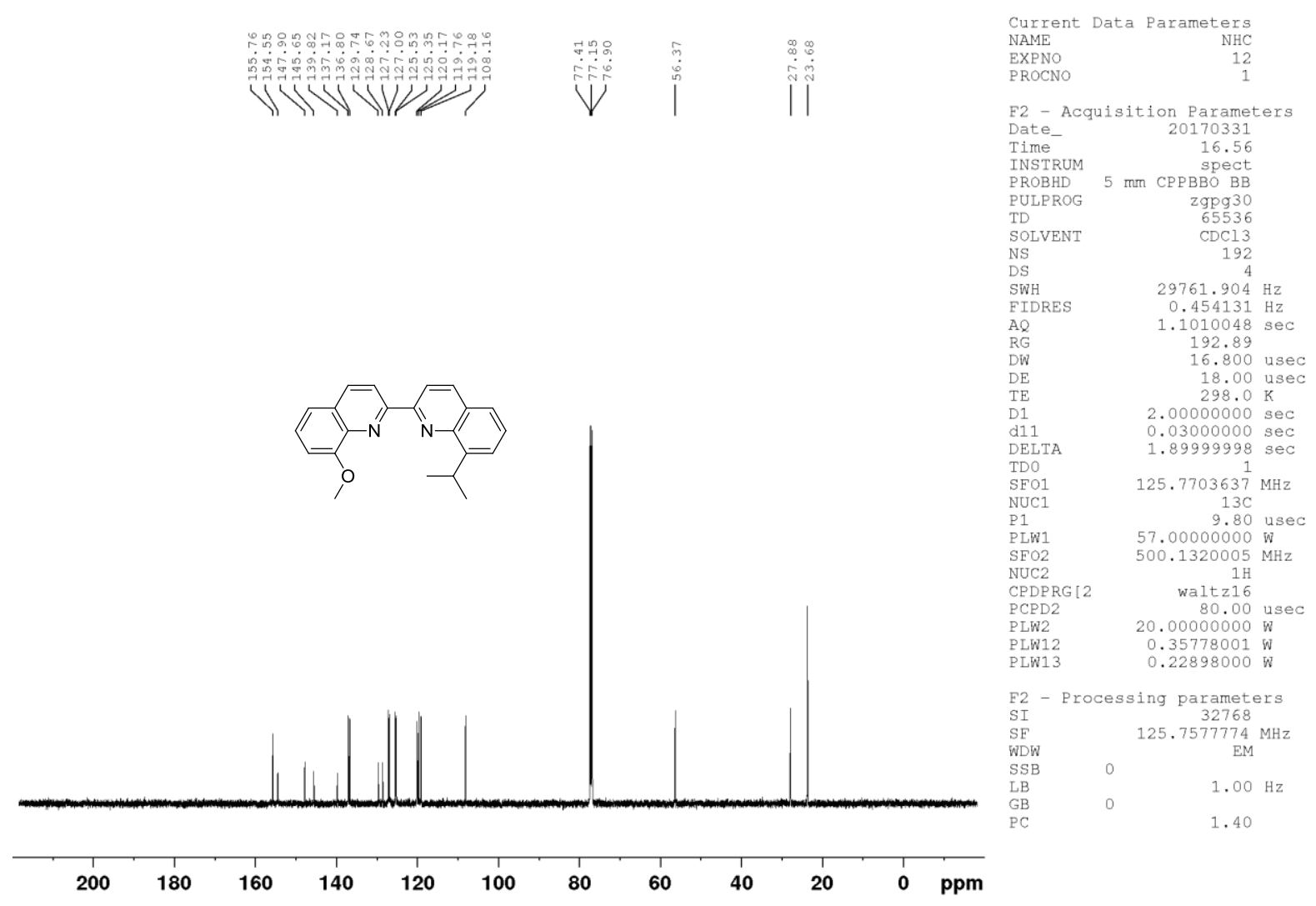


Figure S11. ${ }^{1} \mathrm{H}$ NMR and ${ }^{13} \mathrm{C}$ NMR spectra of $\mathbf{4 b}\left(\mathrm{CDCl}_{3}\right)$

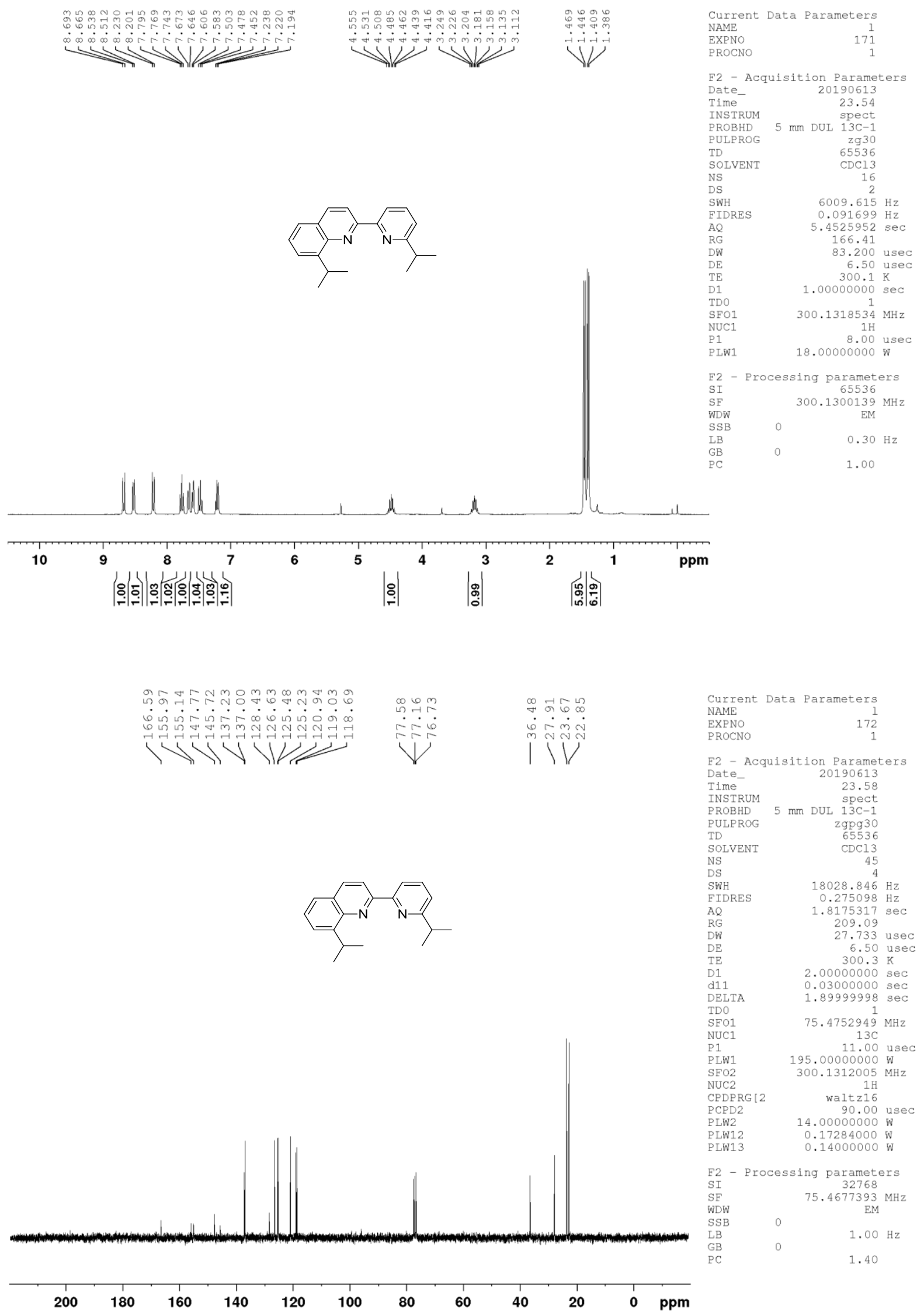


Figure S12. ${ }^{1} \mathrm{H}$ NMR and ${ }^{13} \mathrm{C}$ NMR spectra of $\mathbf{2 f}\left(\mathrm{CDCl}_{3}\right)$

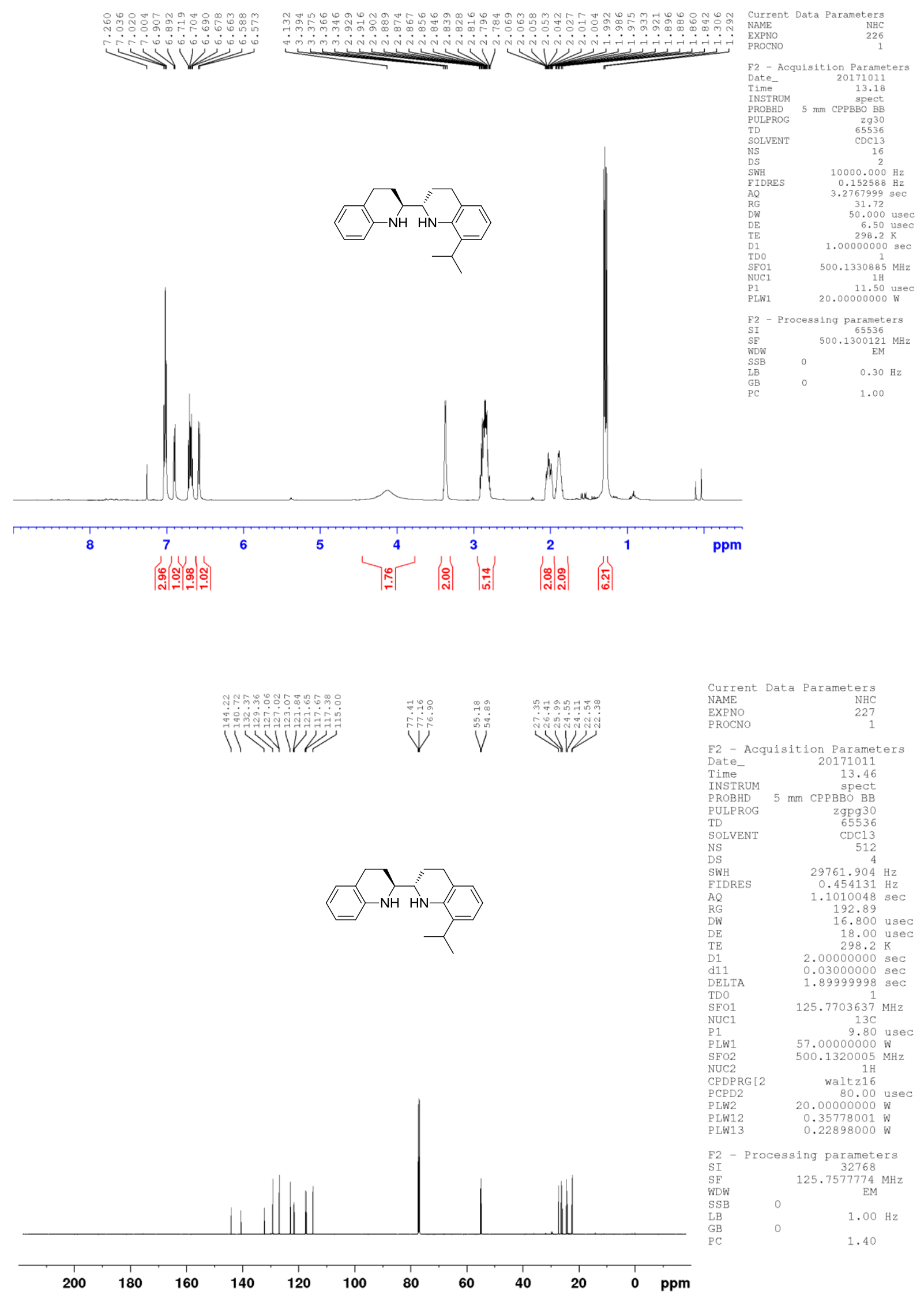


Figure S13. ${ }^{1} \mathrm{H}$ NMR and ${ }^{13} \mathrm{C}$ NMR spectra of $2 \mathrm{~g}\left(\mathrm{CDCl}_{3}\right)$

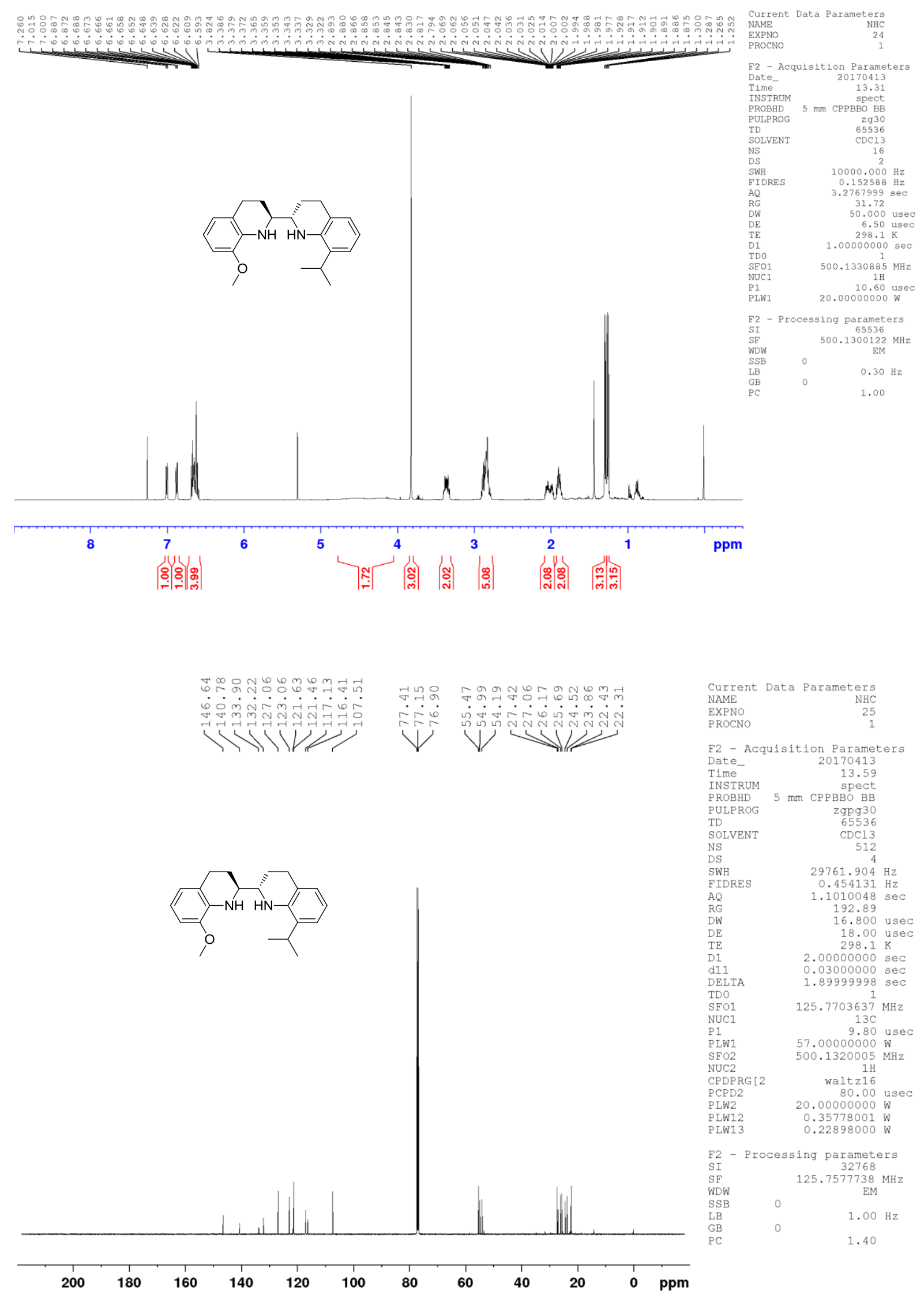


Figure S14. ${ }^{1} \mathrm{H}$ NMR and ${ }^{13} \mathrm{C}$ NMR spectra of $\mathbf{5 b}\left(\mathrm{CDCl}_{3}\right)$

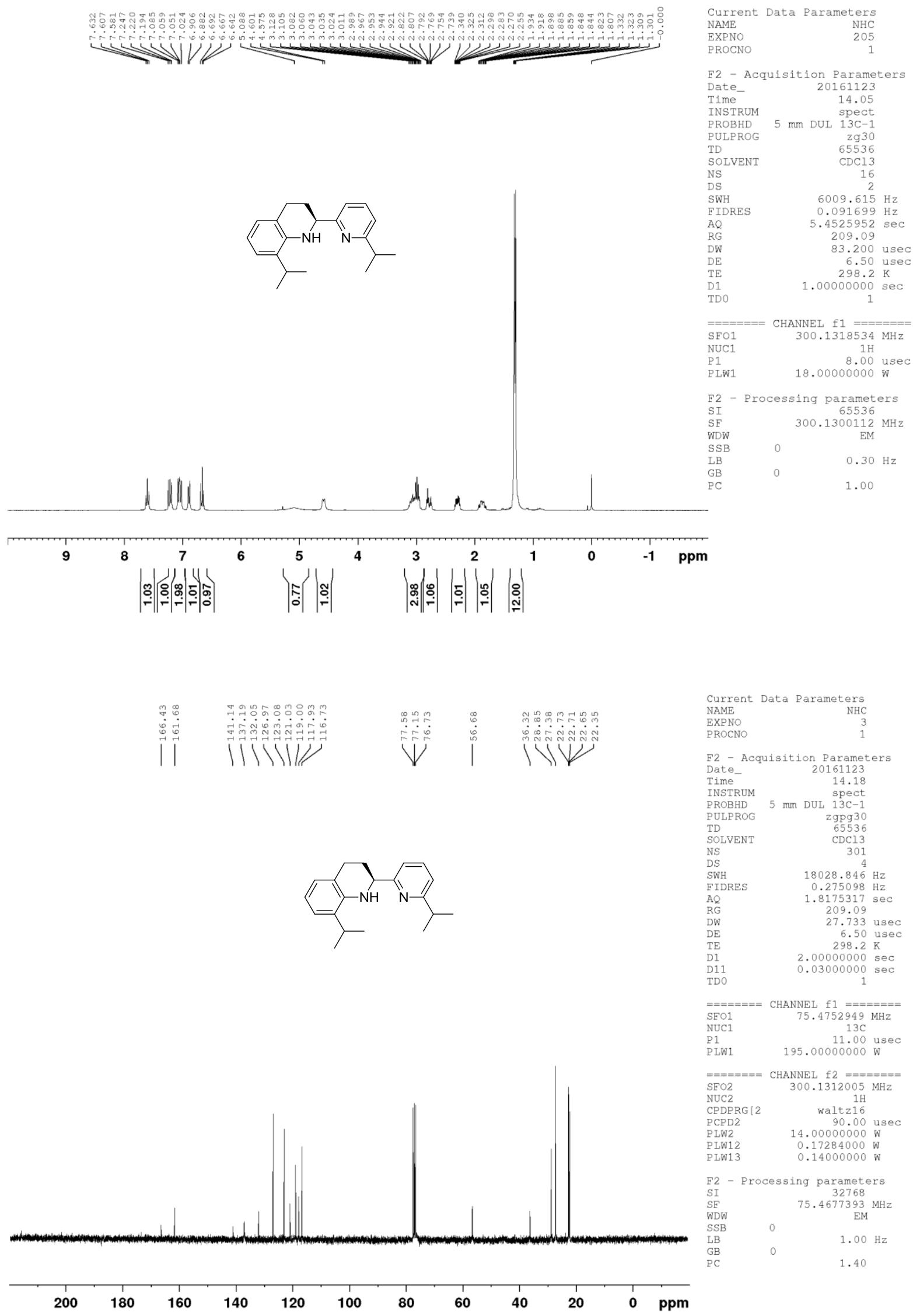


Figure S15. ${ }^{1} \mathrm{H}$ NMR and ${ }^{13} \mathrm{C}$ NMR spectra of $\mathbf{6 a}\left(\mathrm{CDCl}_{3}\right)$

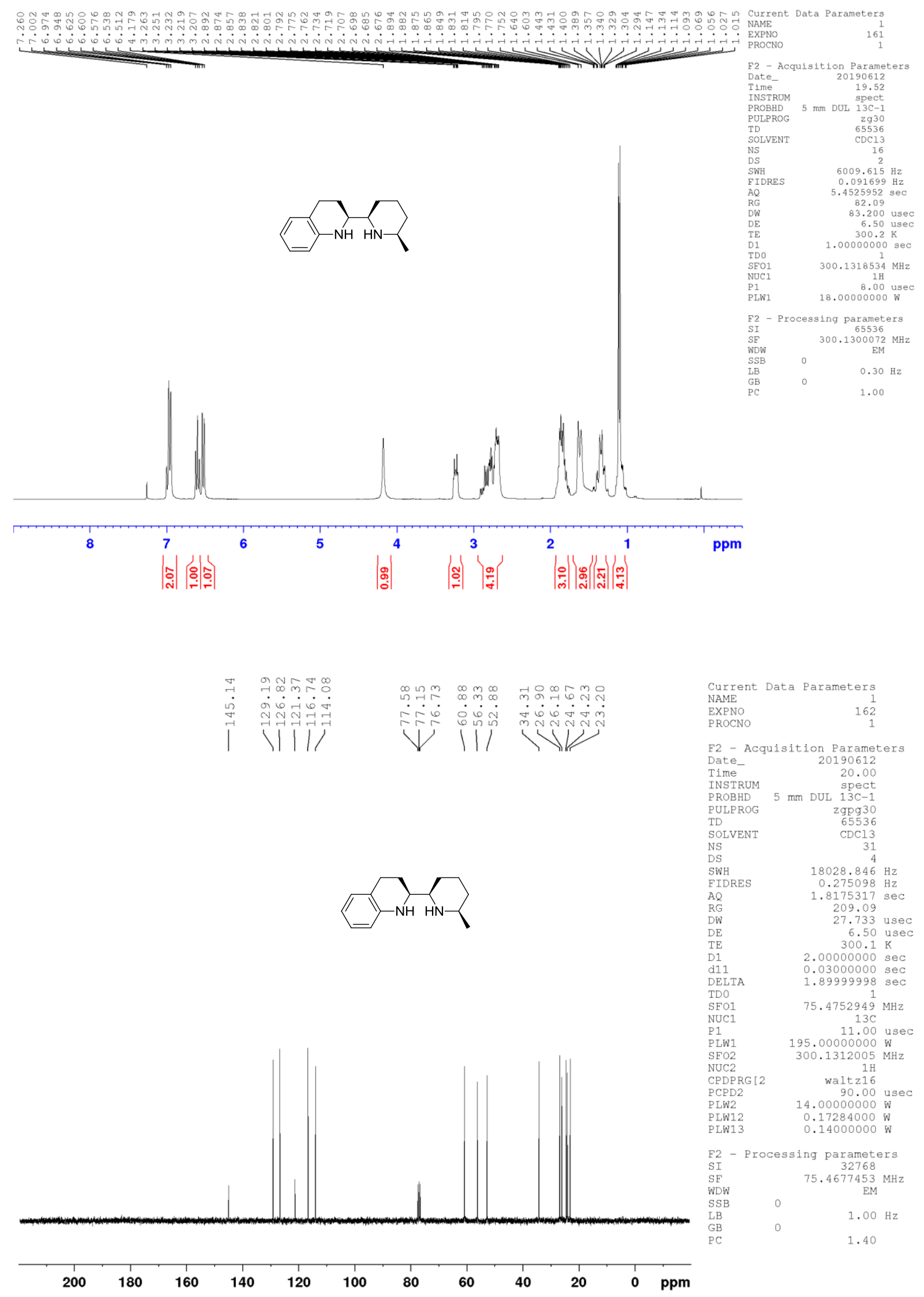


Figure S16. ${ }^{1} \mathrm{H}$ NMR and ${ }^{13} \mathrm{C}$ NMR spectra of $\mathbf{6 b}\left(\mathrm{CDCl}_{3}\right)$

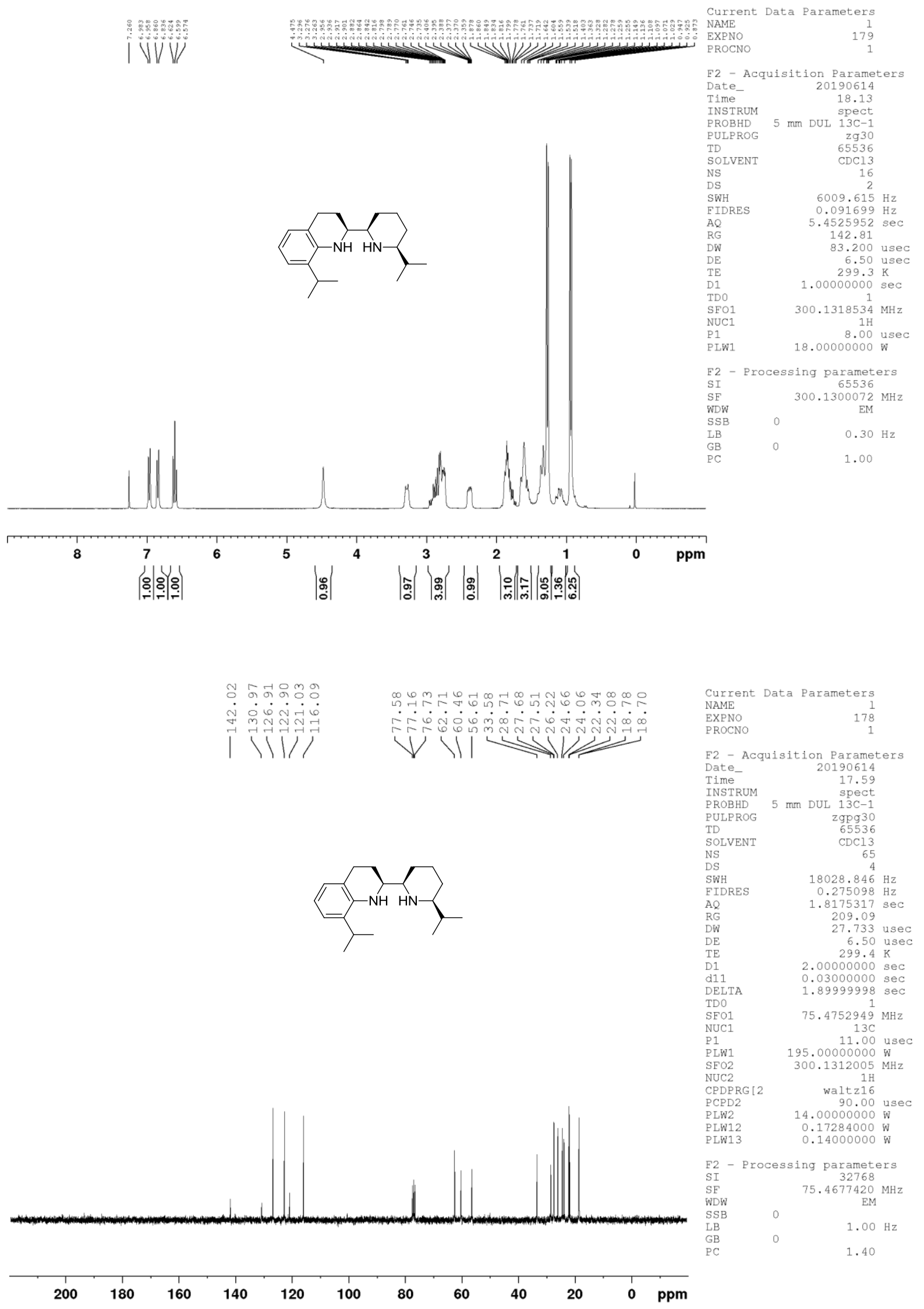


Figure S17. ${ }^{1} \mathrm{H} \mathrm{NMR}$ and ${ }^{13} \mathrm{C}$ NMR spectra of $\mathbf{6 c}\left(\mathrm{CDCl}_{3}\right)$

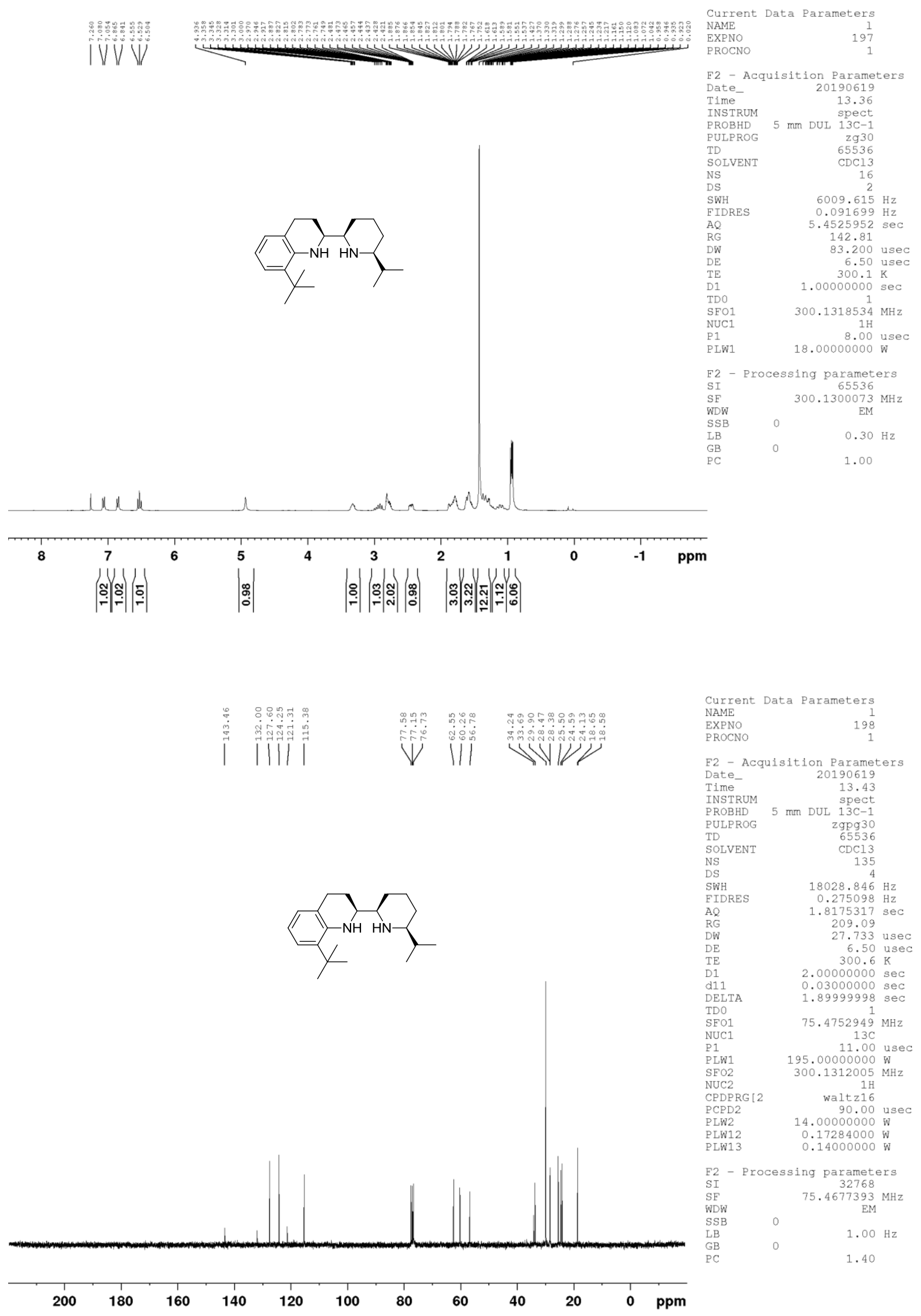


Figure S18. ${ }^{1} \mathrm{H}$ NMR and ${ }^{13} \mathrm{C}$ NMR spectra of $\mathbf{3 d}\left(d^{6}\right.$-DMSO)

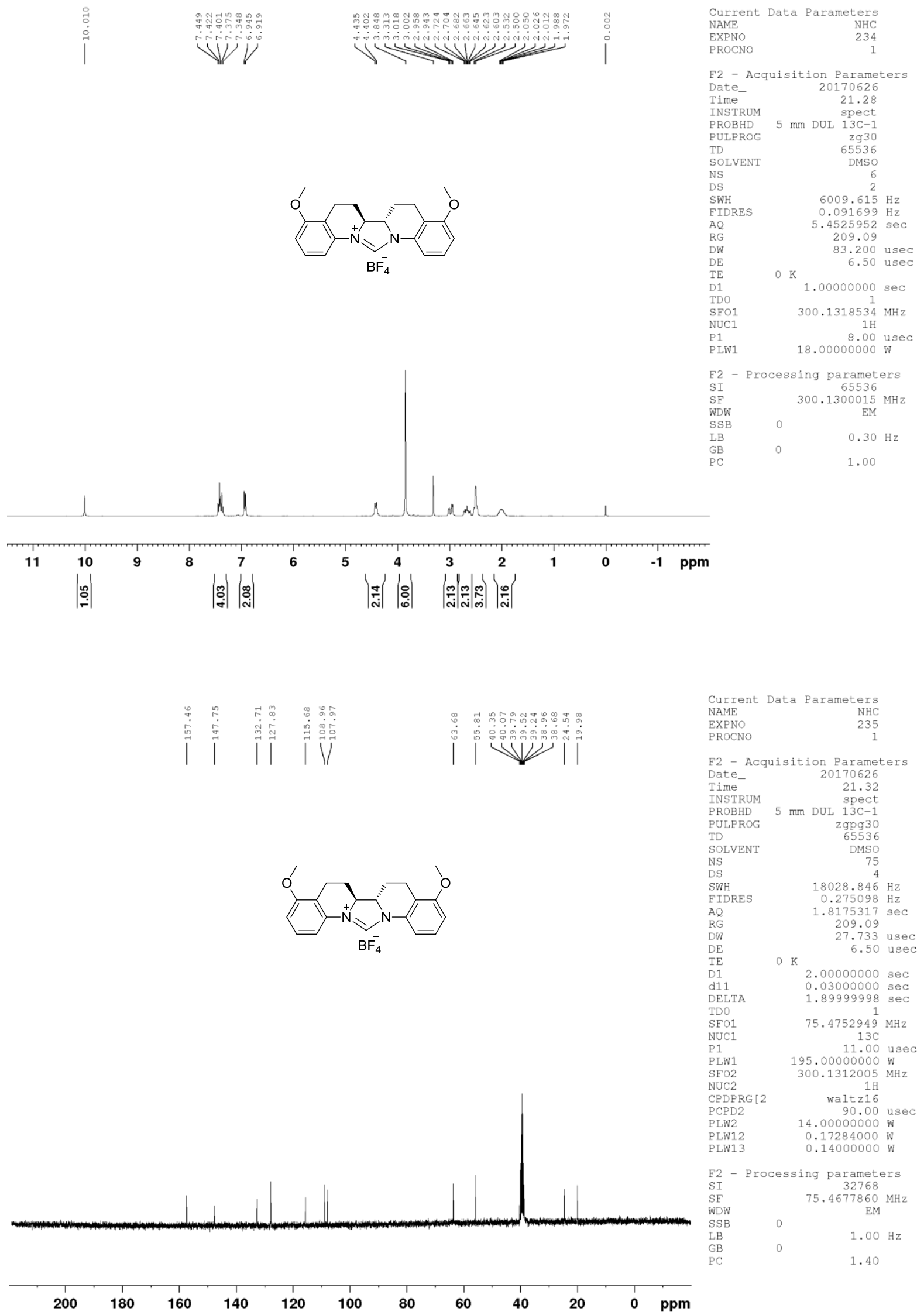


Figure S19. ${ }^{1} \mathrm{H}$ NMR and ${ }^{13} \mathrm{C}$ NMR spectra of $\mathbf{3 e}\left(d^{6}\right.$-DMSO)

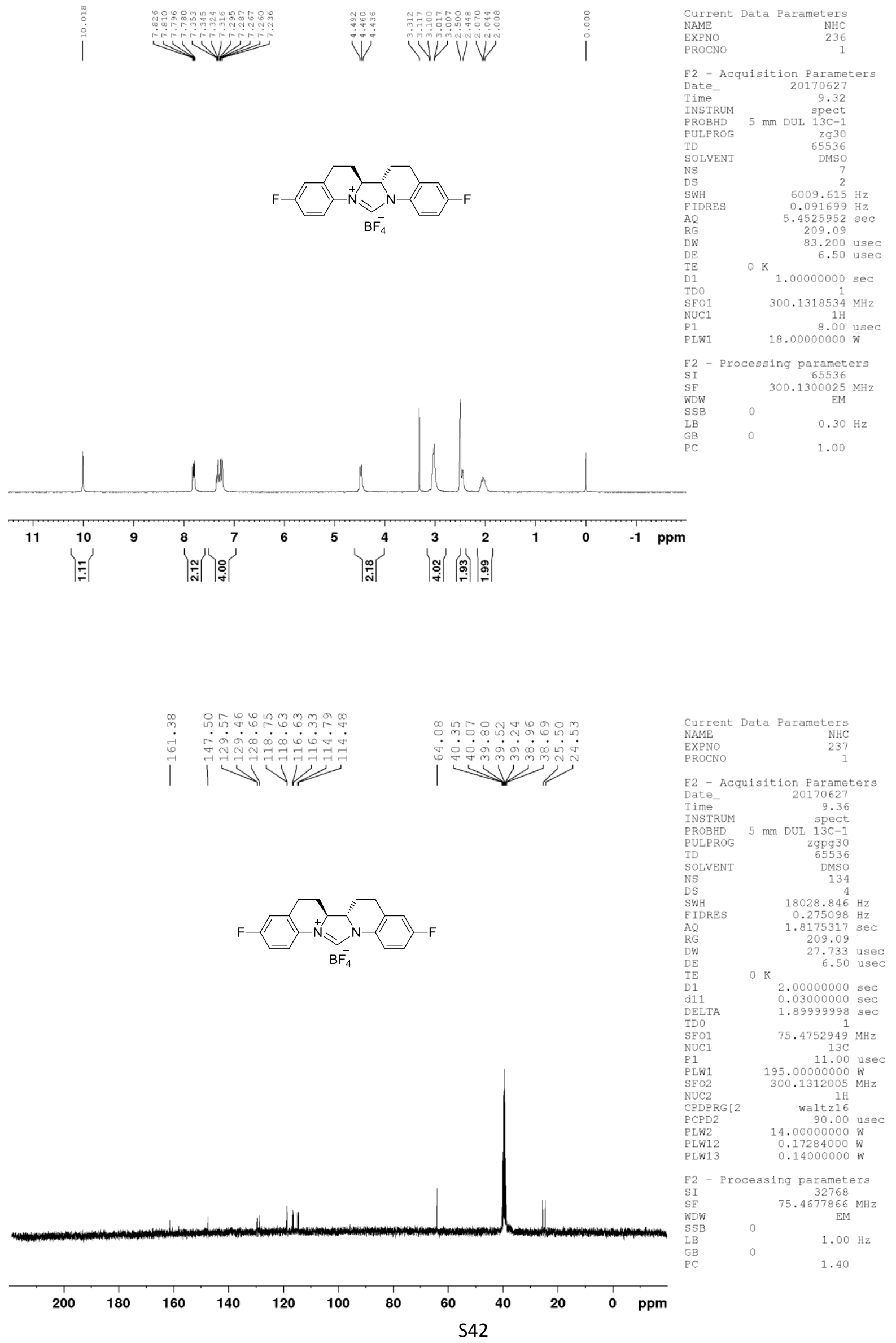


Figure S20. ${ }^{1} \mathrm{H}$ NMR and ${ }^{13} \mathrm{C}$ NMR spectra of $3 \mathbf{f}\left(d^{6}\right.$-DMSO)

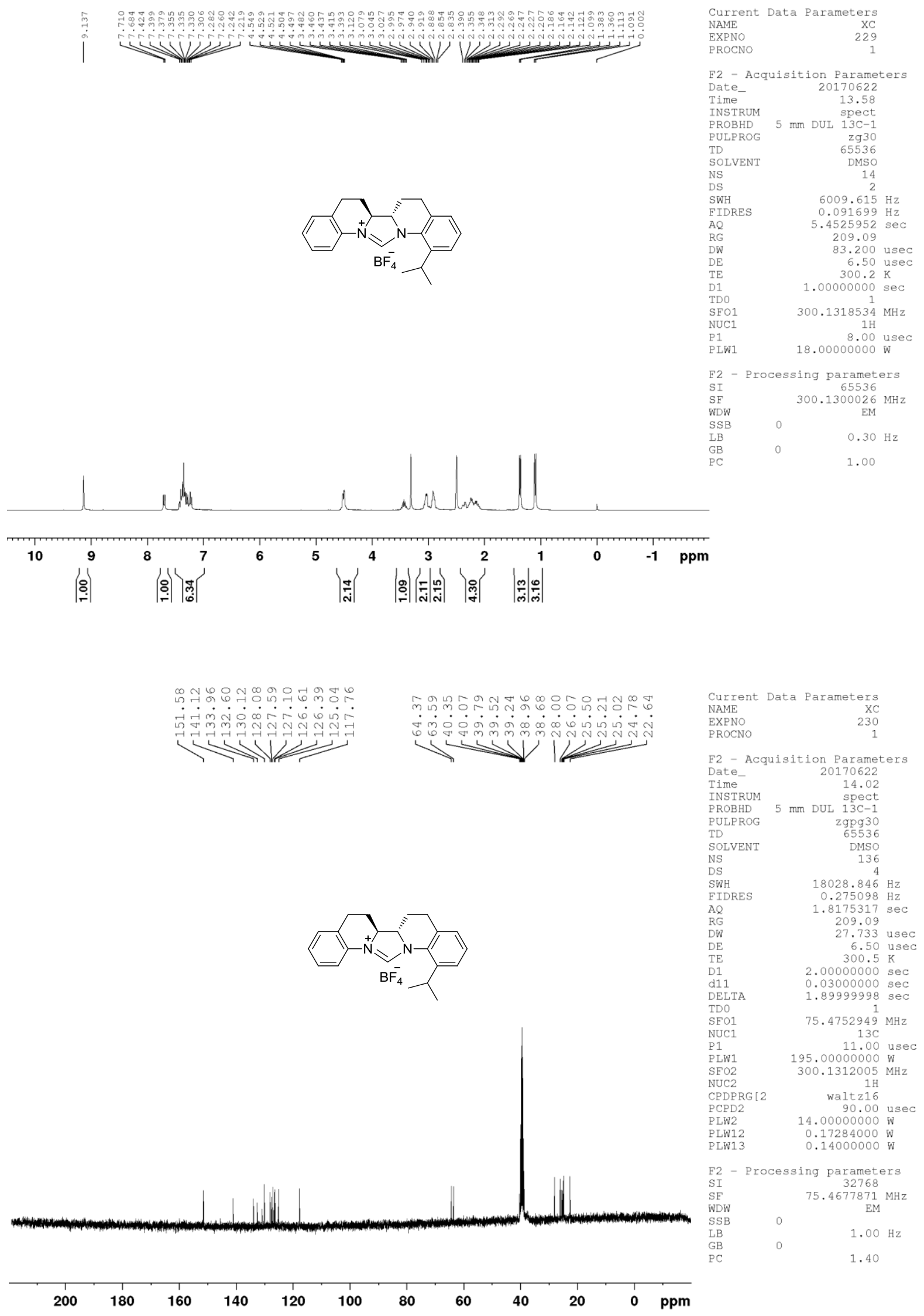


Figure S21. ${ }^{1} \mathrm{H}$ NMR and ${ }^{13} \mathrm{C}$ NMR spectra of $\mathbf{3 g}\left(d^{6}\right.$-DMSO)
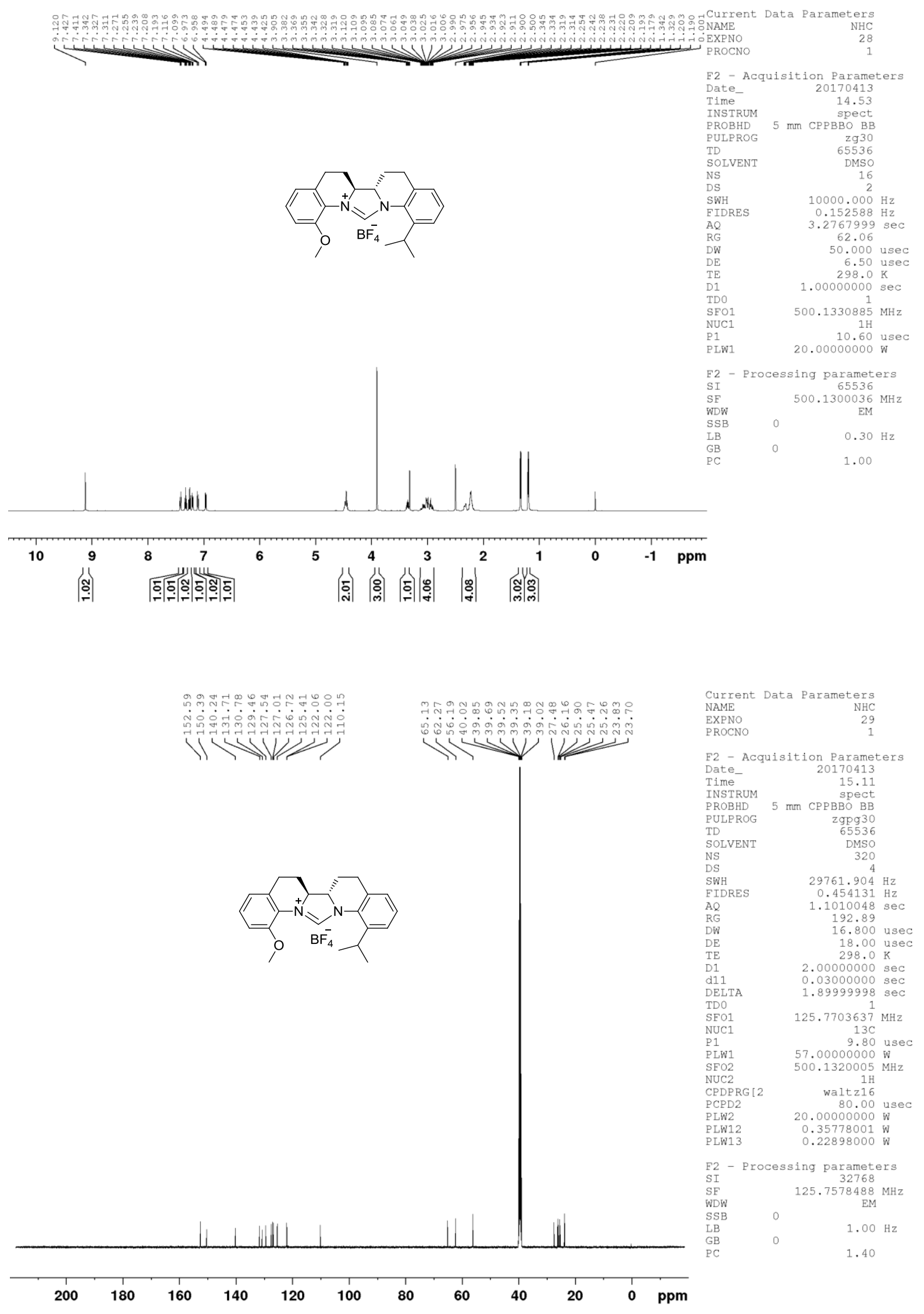
Figure S22. ${ }^{1} \mathrm{H}$ NMR and ${ }^{13} \mathrm{C}$ NMR spectra of $7 \mathrm{a}^{\prime}\left(d^{6}\right.$-DMSO)

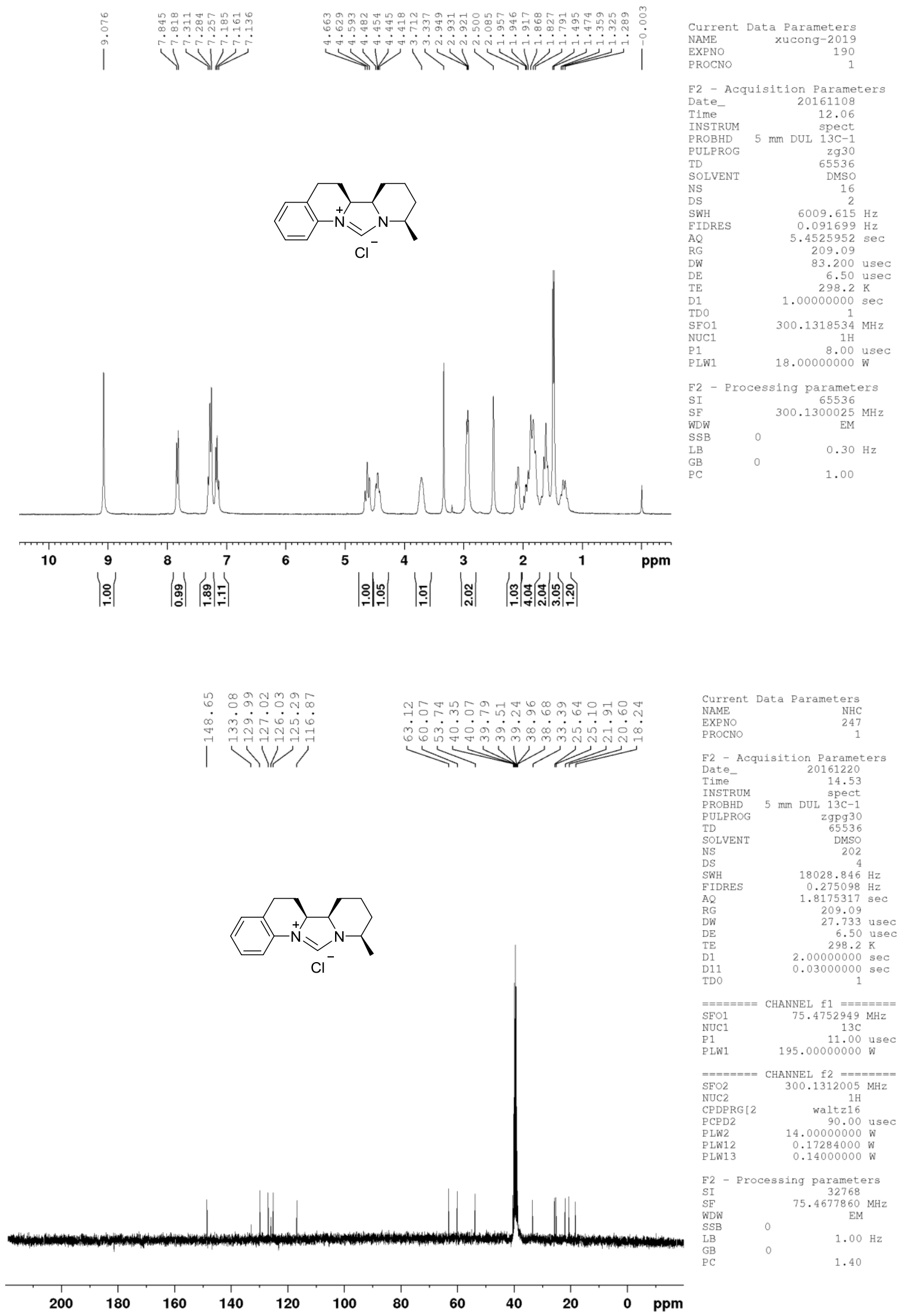


Figure S23. ${ }^{1} \mathrm{H}$ NMR and ${ }^{13} \mathrm{C}$ NMR spectra of $7 \mathbf{a}\left(d^{6}-\mathrm{DMSO}\right)$

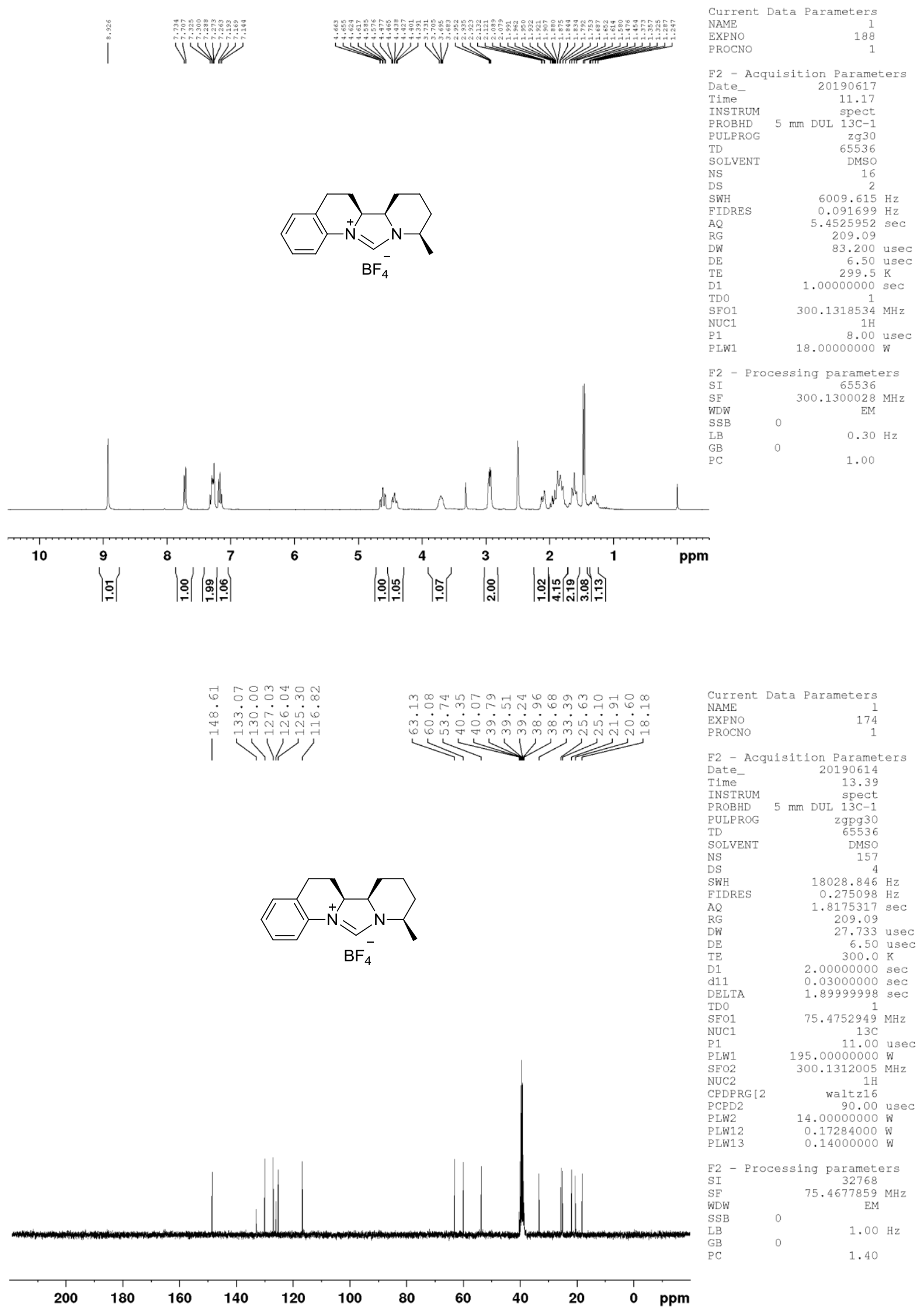


Figure S24. ${ }^{1} \mathrm{H}$ NMR and ${ }^{13} \mathrm{C}$ NMR spectra of $7 \mathrm{~b}\left(d^{6}\right.$-DMSO)

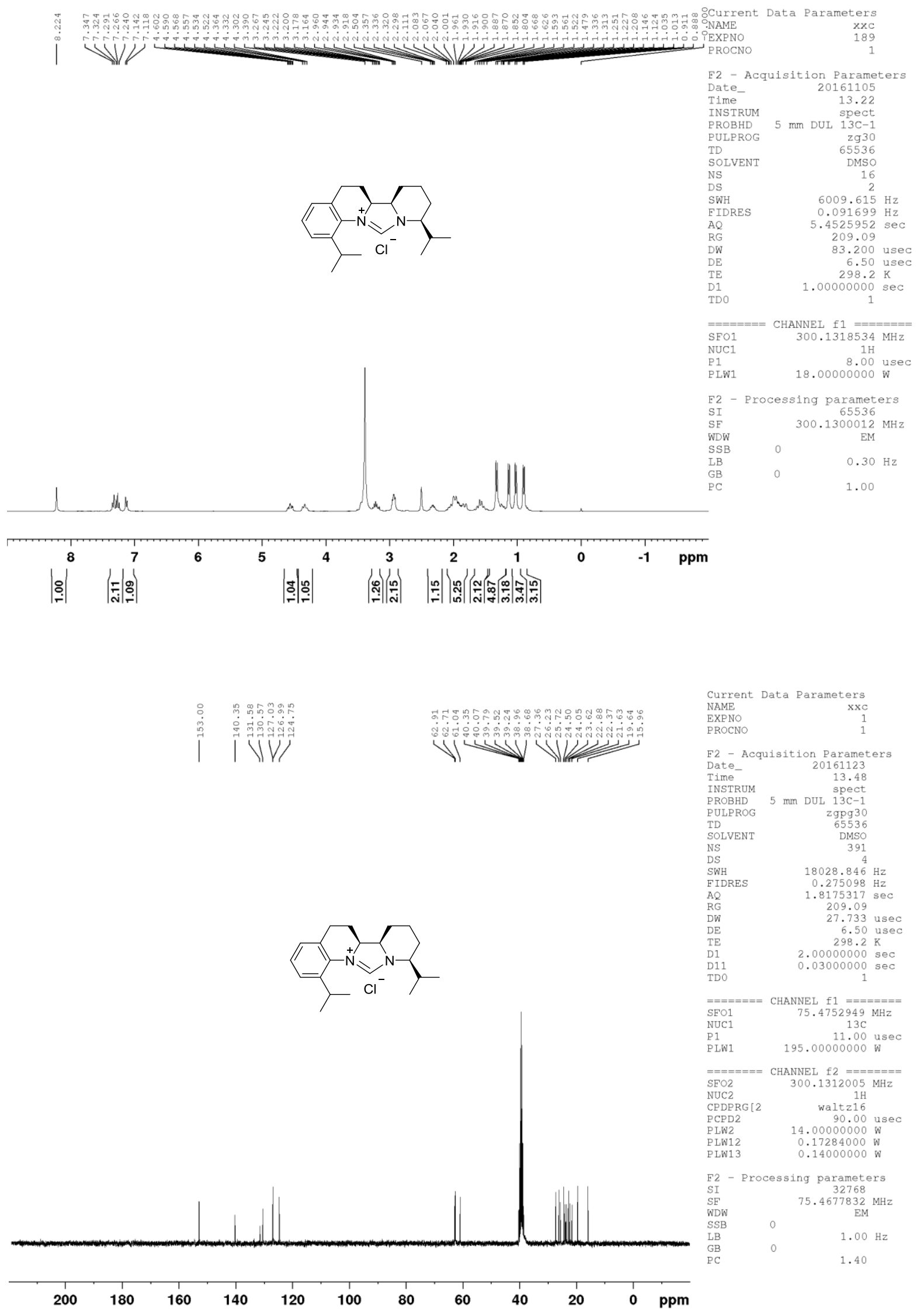


Figure S25. ${ }^{1} \mathrm{H}$ NMR and ${ }^{13} \mathrm{C}$ NMR spectra of $7 \mathrm{c}\left(d^{6}\right.$-DMSO)

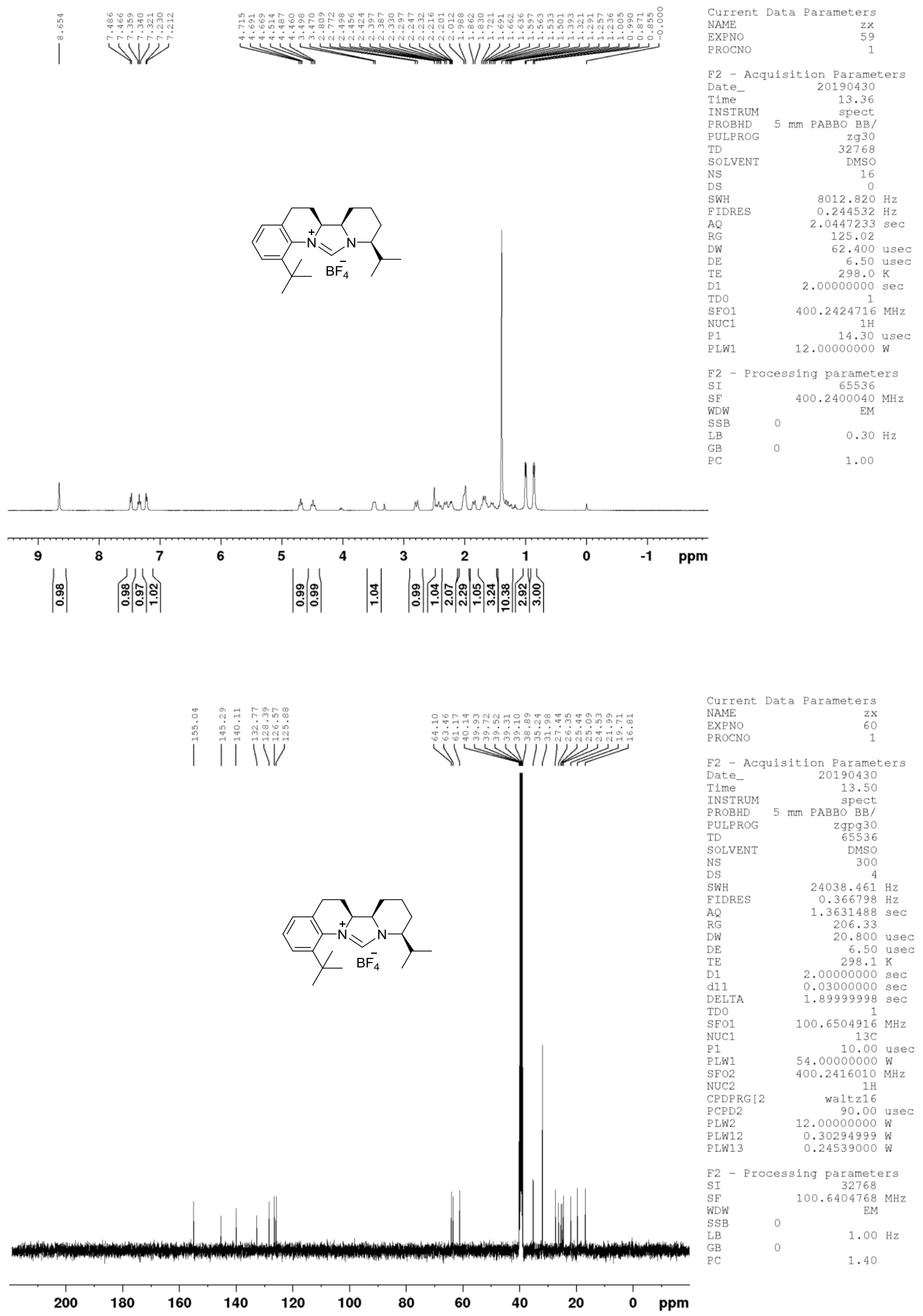


Figure S26. ${ }^{1} \mathrm{H}$ NMR and ${ }^{13} \mathrm{C}$ NMR spectra of $7 \mathrm{~d}\left(\mathrm{CDCl}_{3}\right)$

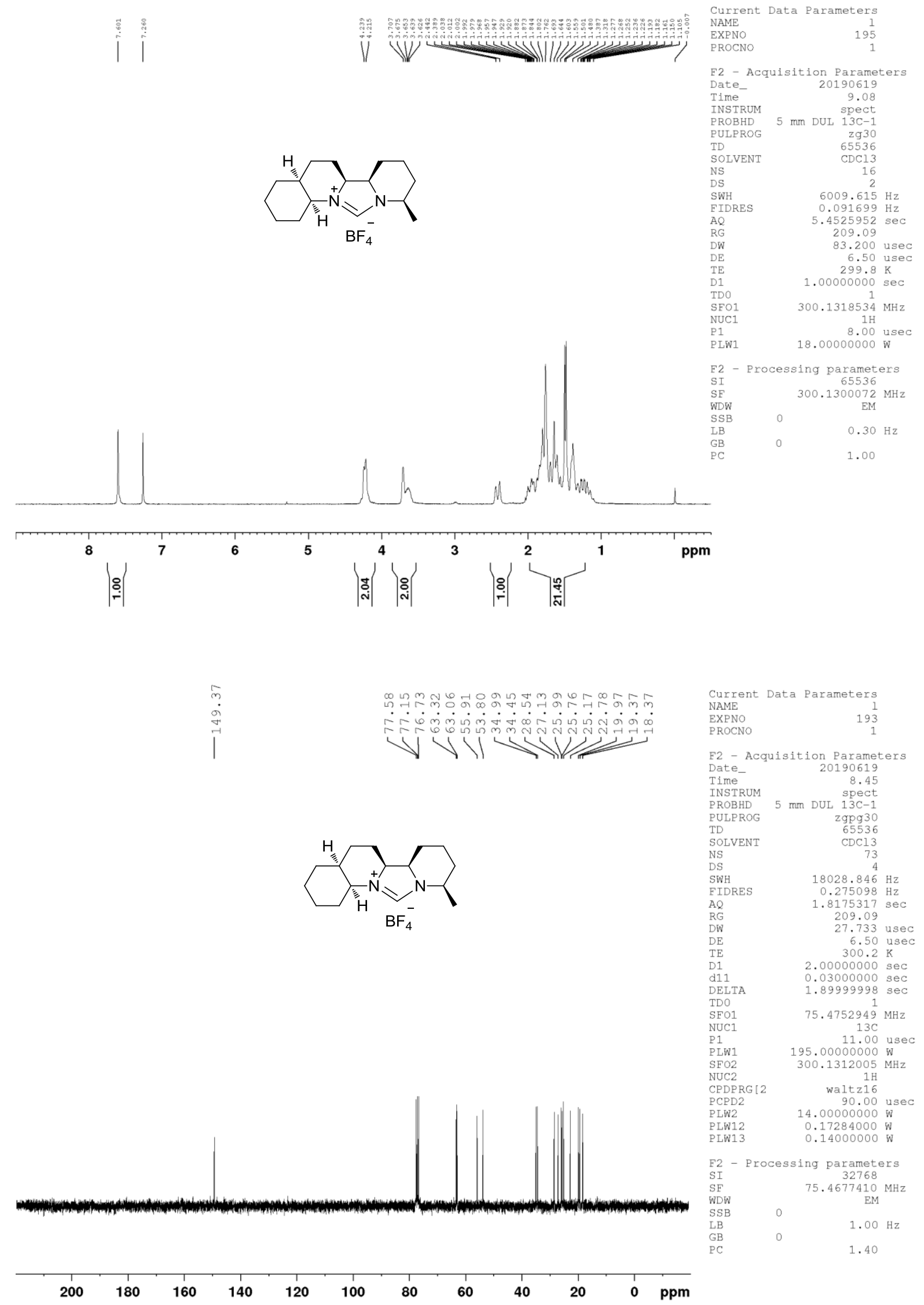


Figure S27. ${ }^{1} \mathrm{H}$ NMR and ${ }^{13} \mathrm{C}$ NMR spectra of $8 \mathrm{a}\left(\mathrm{CDCl}_{3}\right)$
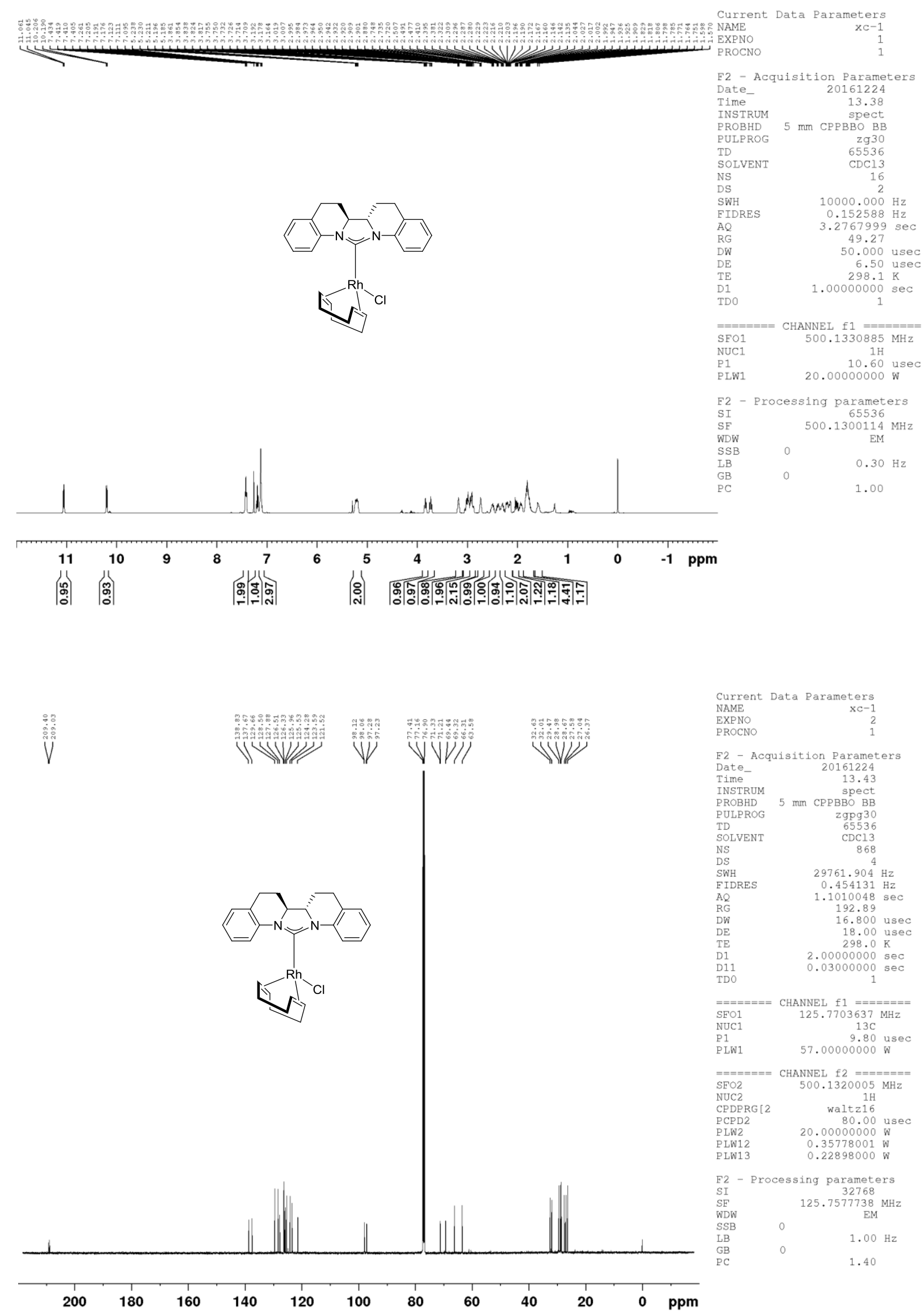
Figure S28. ${ }^{1} \mathrm{H}$ NMR and ${ }^{13} \mathrm{C}$ NMR spectra of $\mathbf{8 b}\left(\mathrm{CDCl}_{3}\right)$
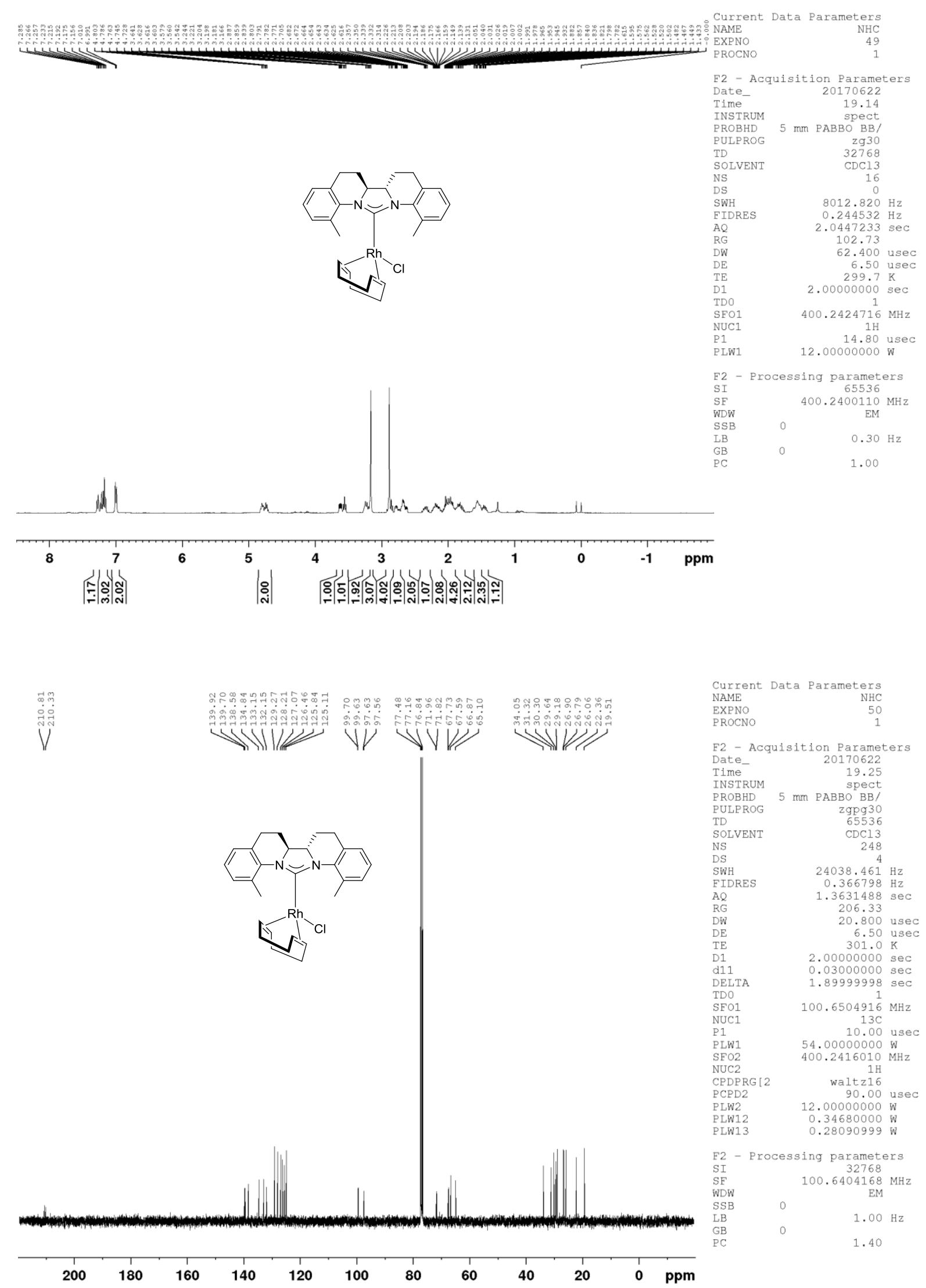
Figure S29. ${ }^{1} \mathrm{H}$ NMR and ${ }^{13} \mathrm{C}$ NMR spectra of $\mathbf{8 c}\left(\mathrm{CDCl}_{3}\right)$
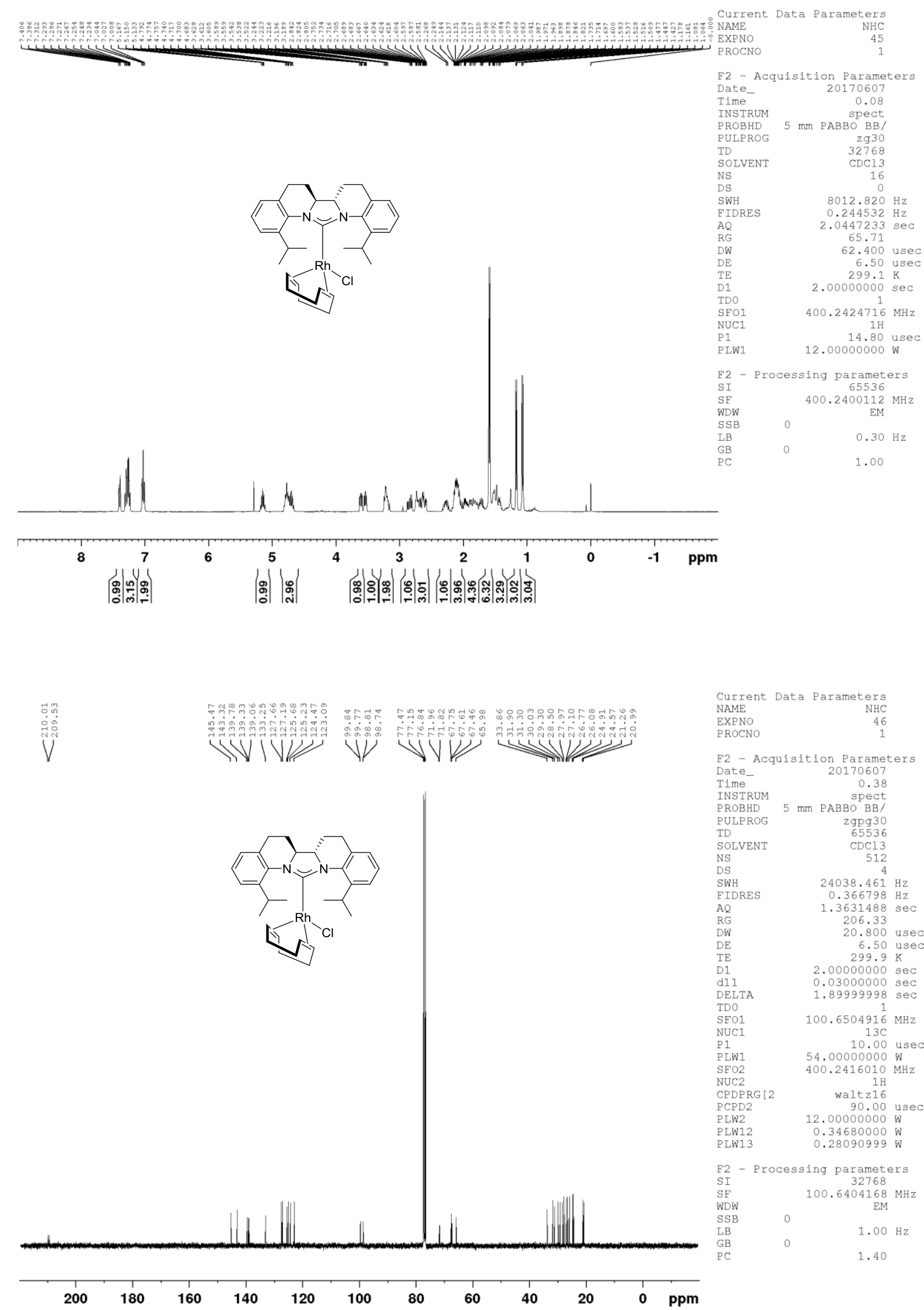
Figure S30. ${ }^{1} \mathrm{H}$ NMR and ${ }^{13} \mathrm{C}$ NMR spectra of $\mathbf{8 d}\left(\mathrm{CDCl}_{3}\right)$
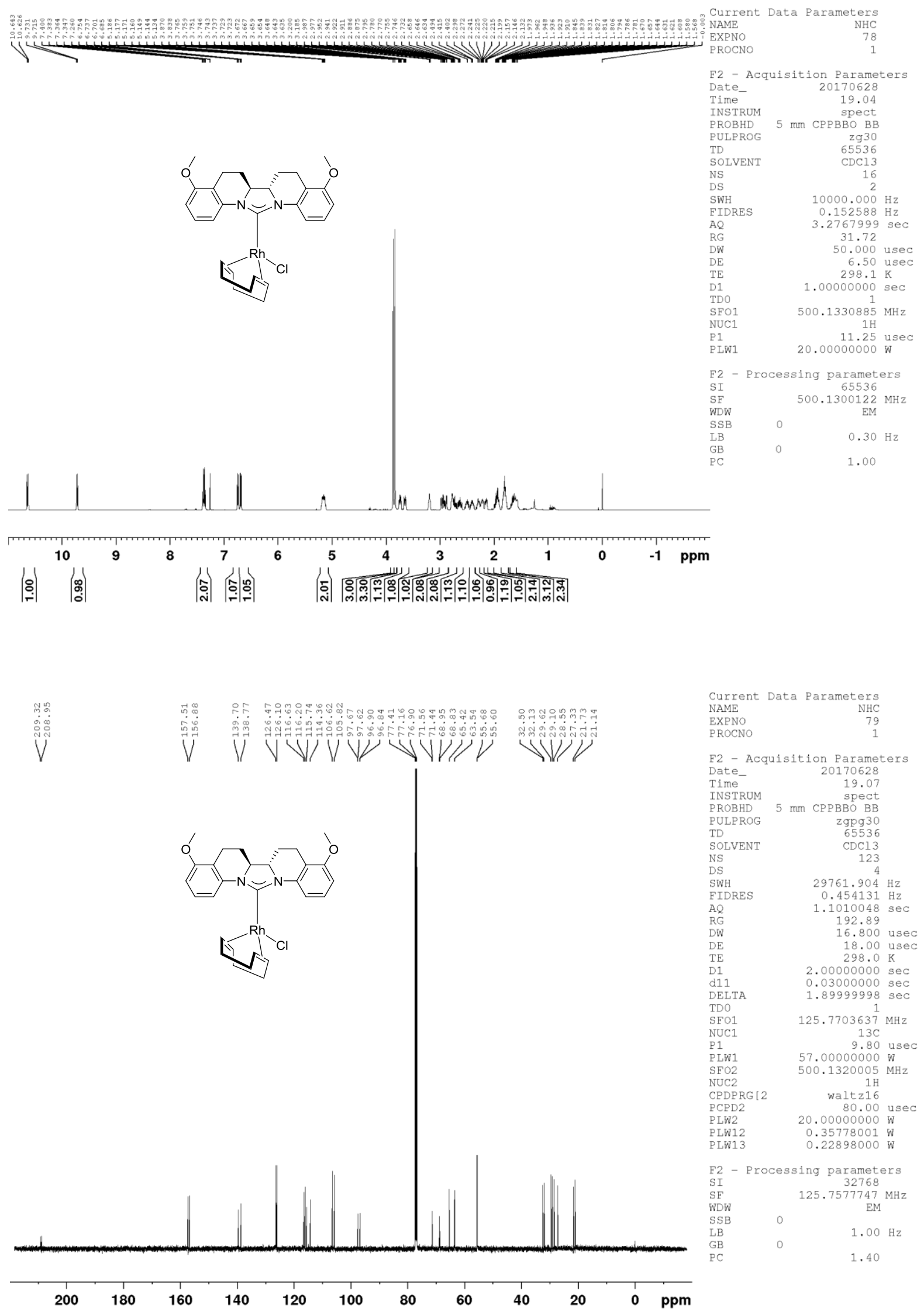
Figure S31. ${ }^{1} \mathrm{H}$ NMR and ${ }^{13} \mathrm{C}$ NMR spectra of $\mathbf{8 e}\left(\mathrm{CDCl}_{3}\right)$
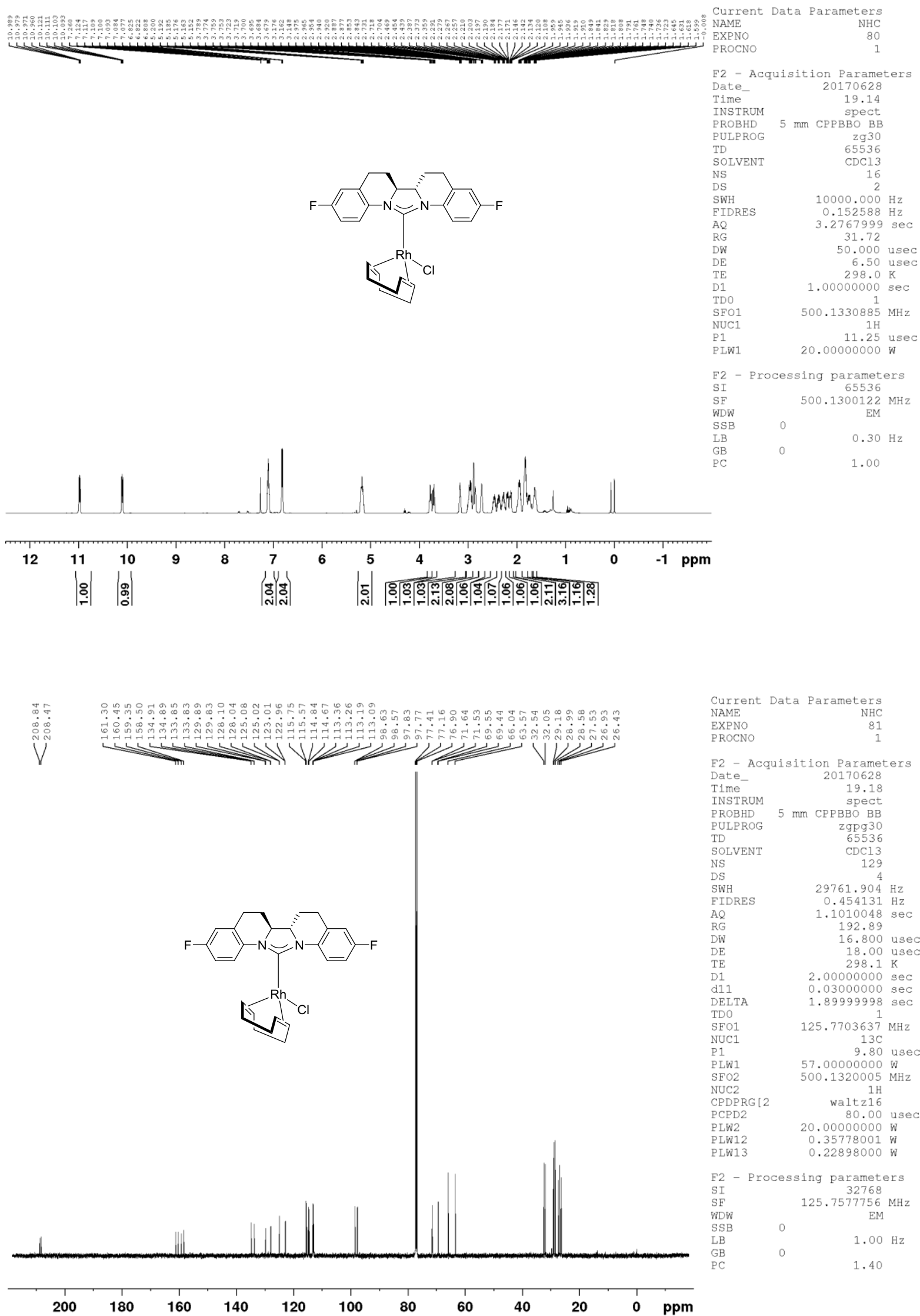
Figure S32. ${ }^{1} \mathrm{H}$ NMR and ${ }^{13} \mathrm{C}$ NMR spectra of $\mathbf{8 f}\left(\mathrm{CDCl}_{3}\right)$
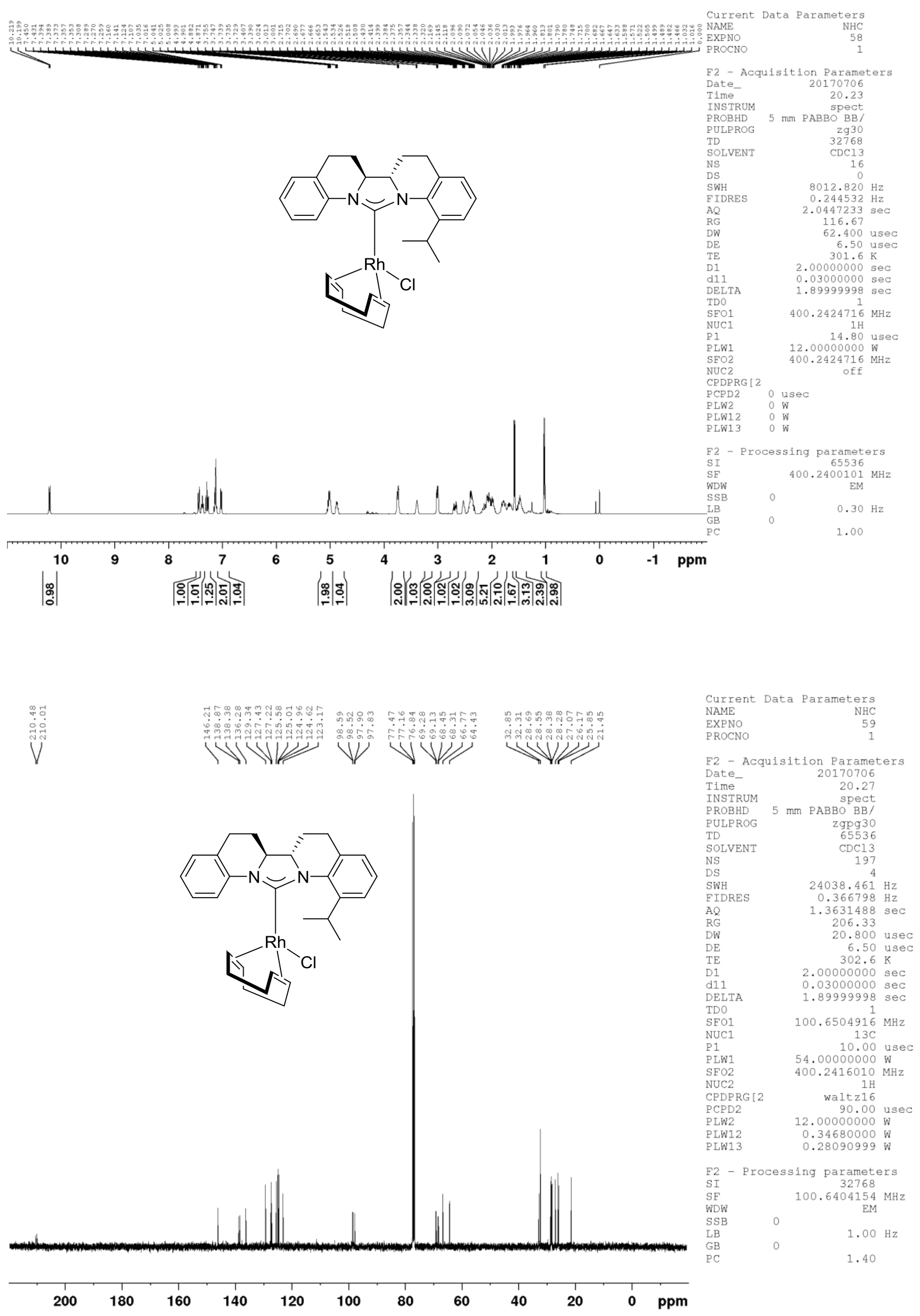
Figure S33. ${ }^{1} \mathrm{H}$ NMR and ${ }^{13} \mathrm{C}$ NMR spectra of $\mathbf{8 g}\left(\mathrm{CDCl}_{3}\right)$
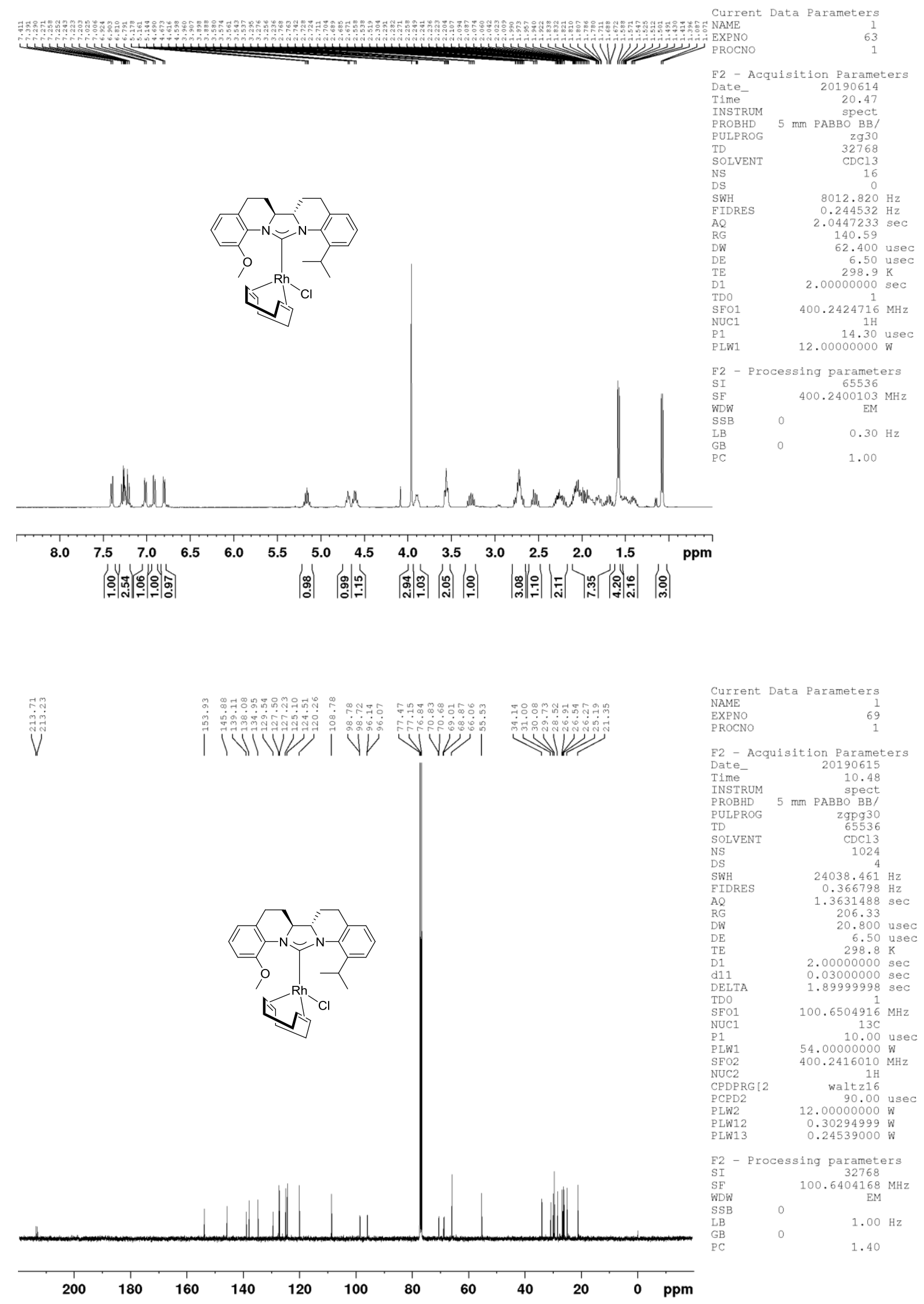
Figure S34. ${ }^{1} \mathrm{H}$ NMR and ${ }^{13} \mathrm{C}$ NMR spectra of $\mathbf{1 0 a}\left(\mathrm{CDCl}_{3}\right)$

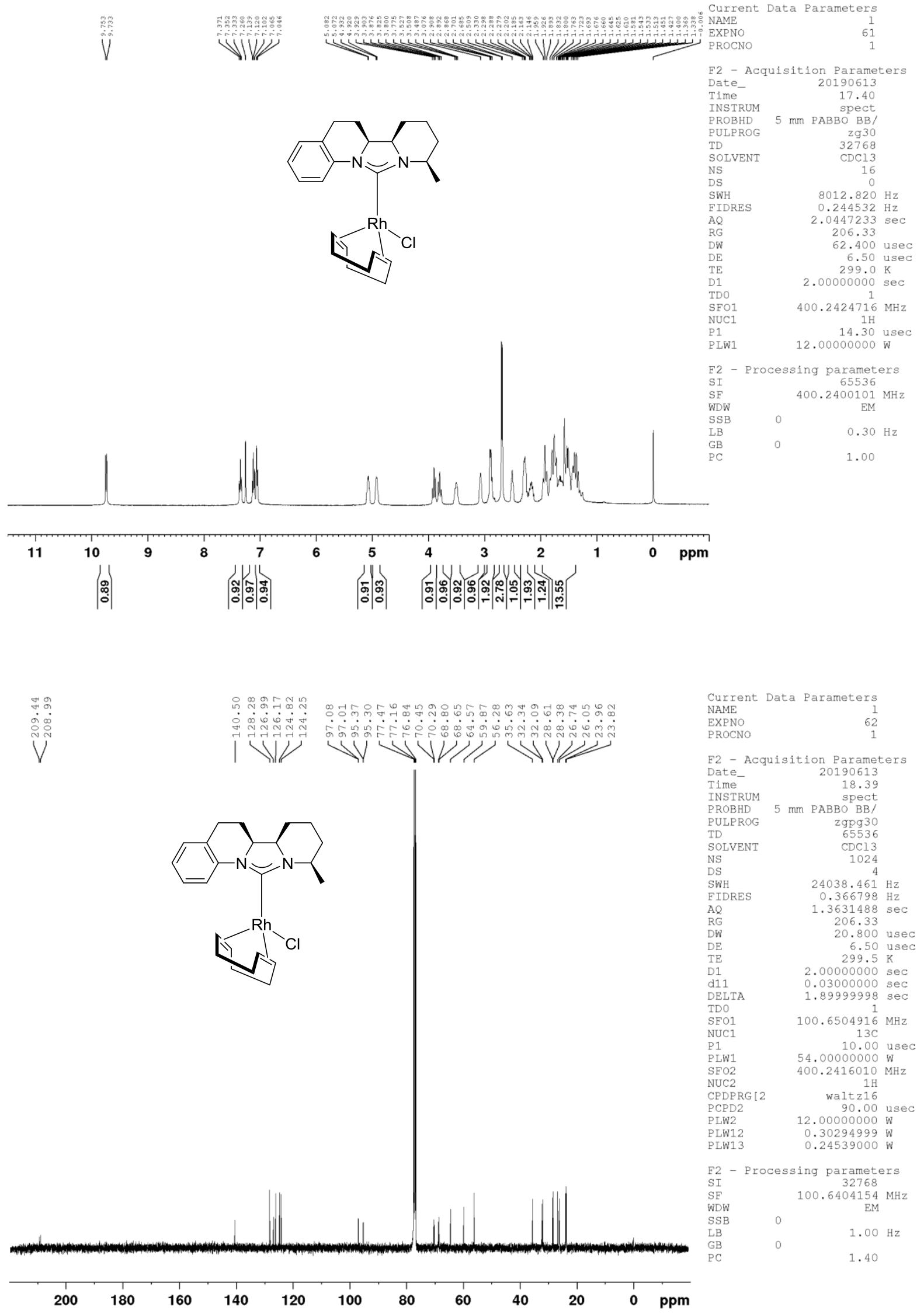


Figure S35. ${ }^{1} \mathrm{H}$ NMR and ${ }^{13} \mathrm{C}$ NMR spectra of $\mathbf{1 0 b}\left(\mathrm{CDCl}_{3}\right)$

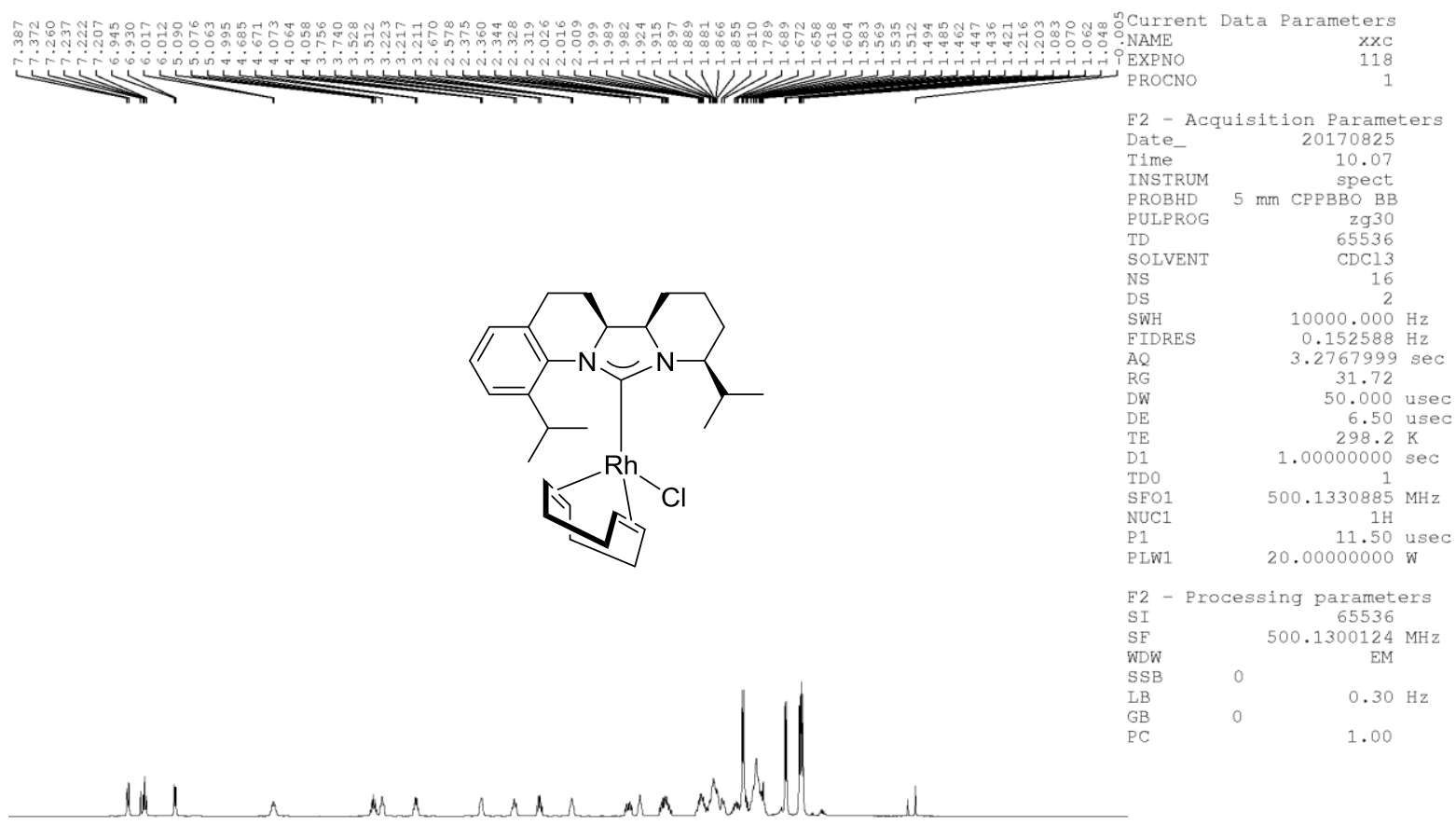

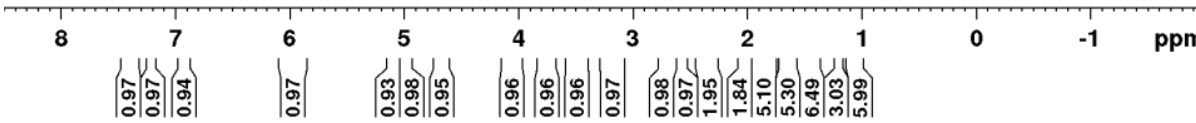

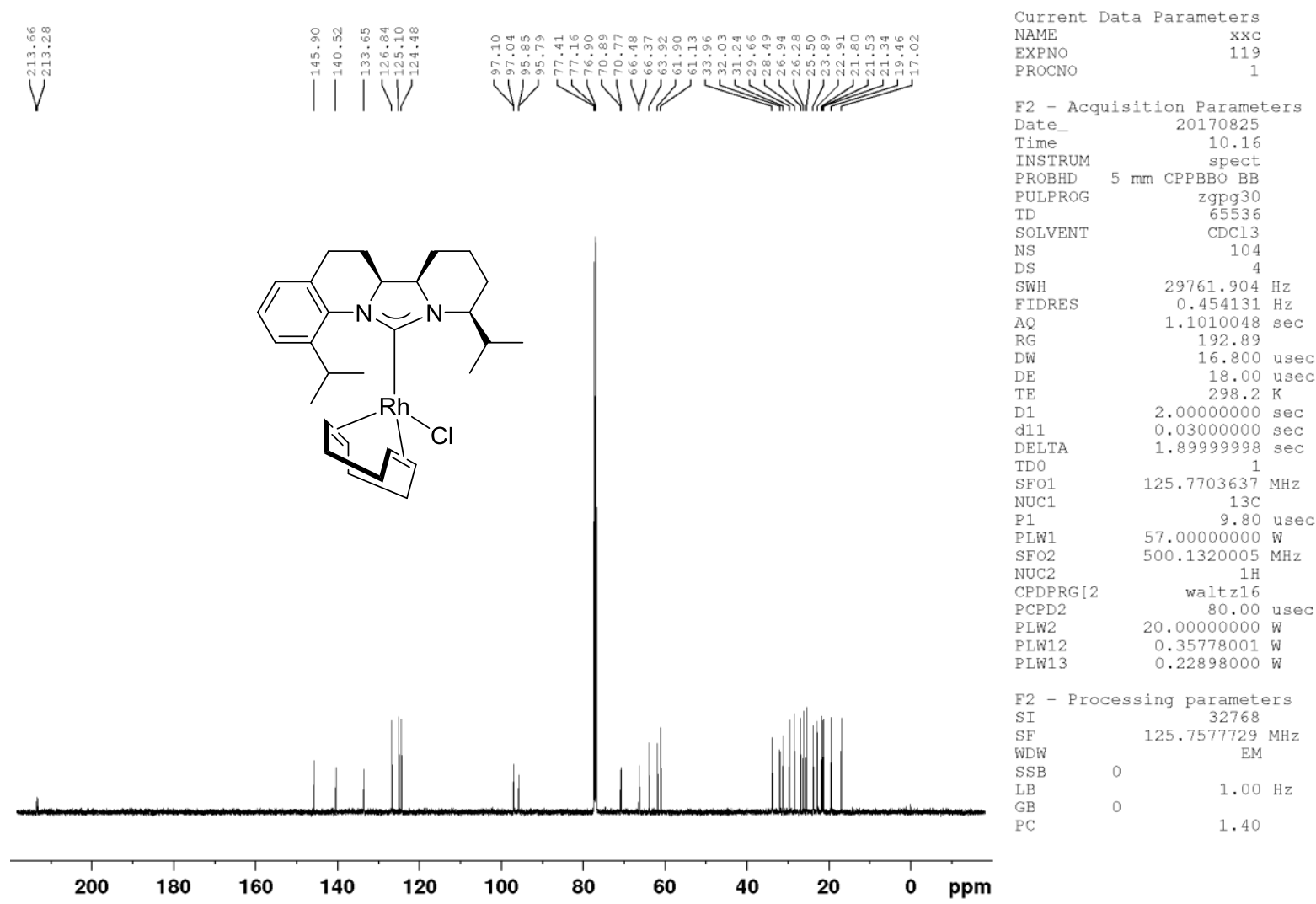


Figure S36. ${ }^{1} \mathrm{H} \mathrm{NMR}$ and ${ }^{13} \mathrm{C} \mathrm{NMR}$ spectra of $\mathbf{1 0 c}\left(\mathrm{CDCl}_{3}\right)$

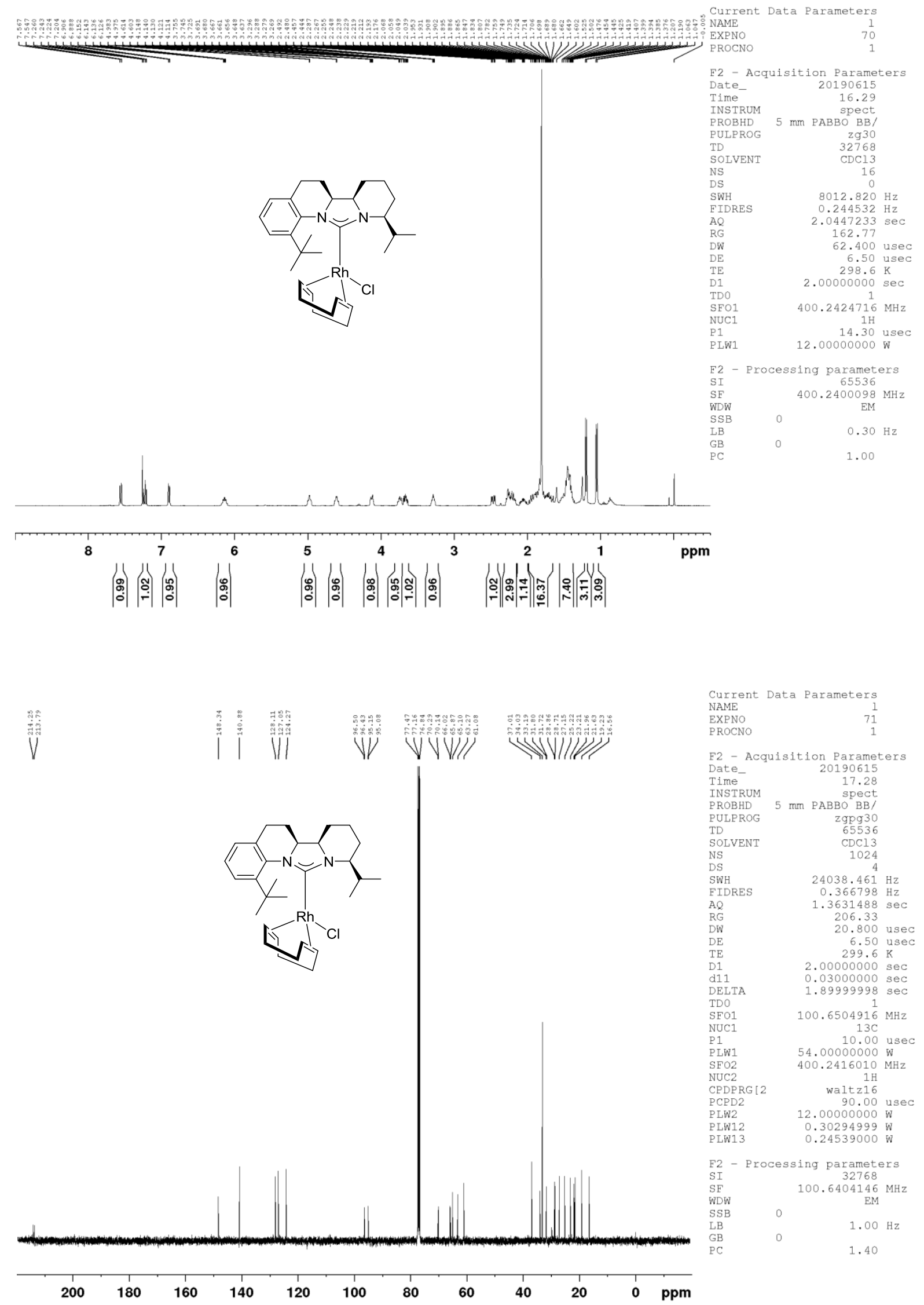


Figure S37. ${ }^{1} \mathrm{H}$ NMR and ${ }^{13} \mathrm{C}$ NMR spectra of $10 d\left(\mathrm{CDCl}_{3}\right)$

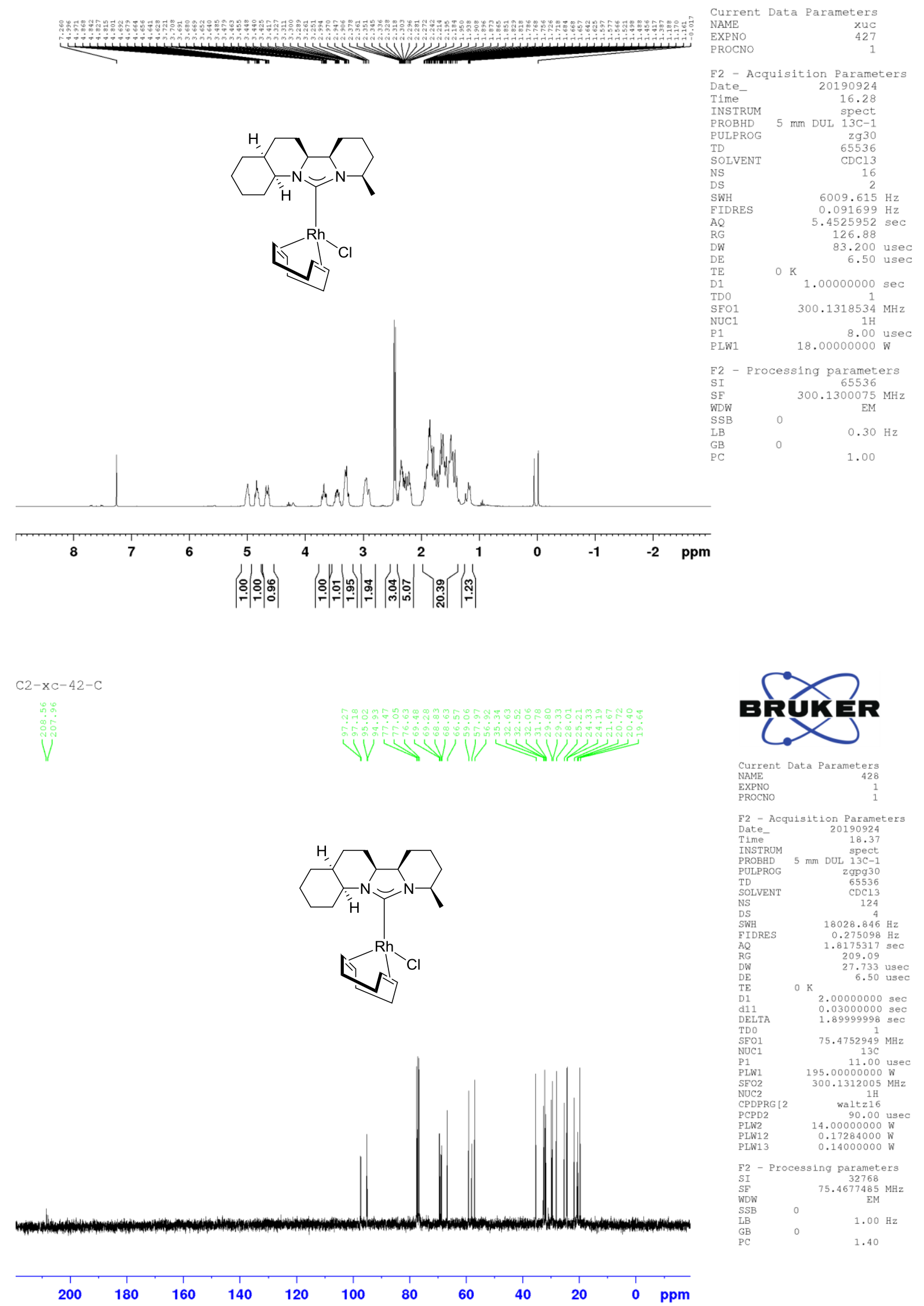


Figure S38. ${ }^{1} \mathrm{H}$ NMR and ${ }^{13} \mathrm{C}$ NMR spectra of $9 a\left(\mathrm{CDCl}_{3}\right)$
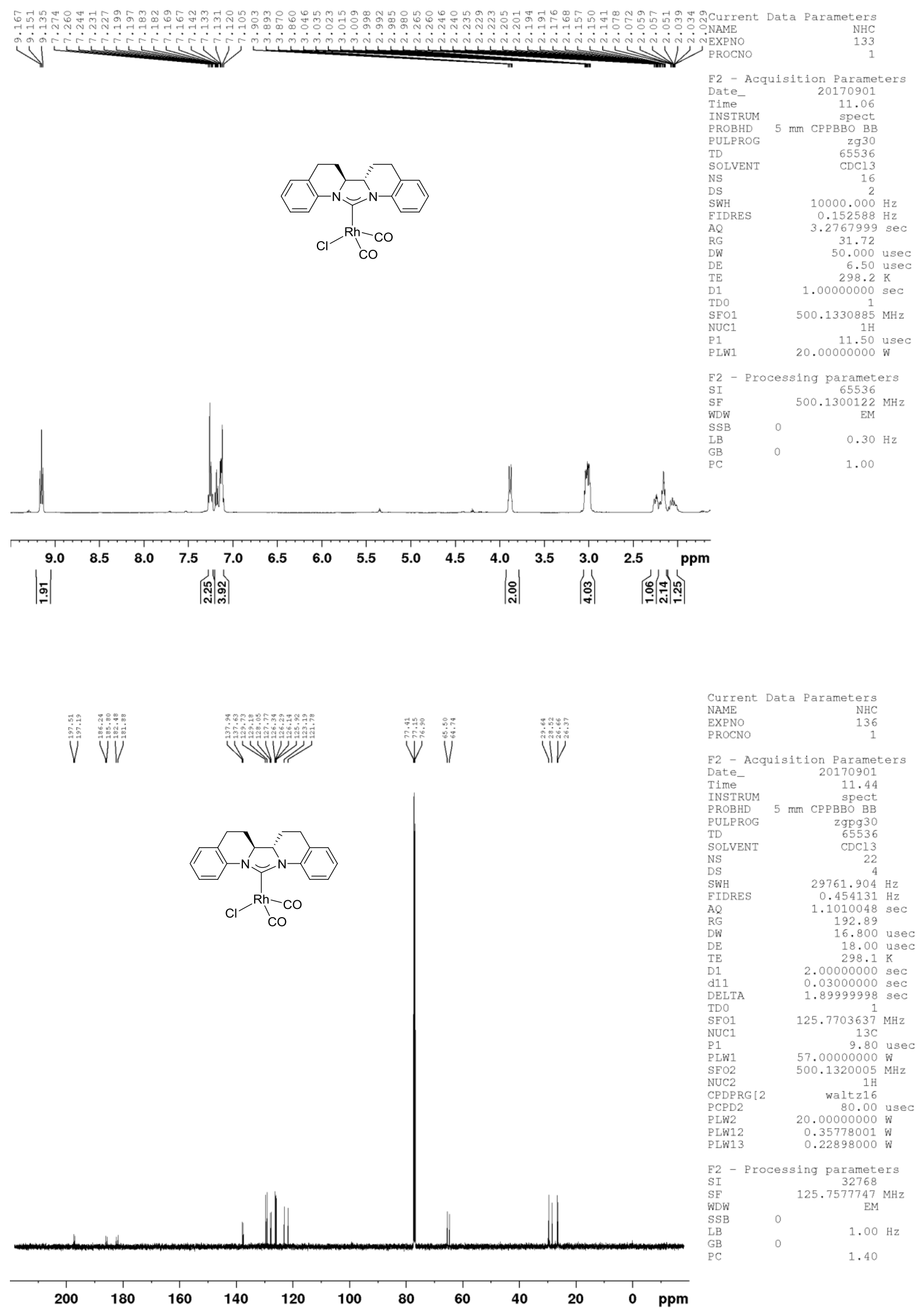
Figure S39. ${ }^{1} \mathrm{H}$ NMR and ${ }^{13} \mathrm{C}$ NMR spectra of $\mathbf{9 b}\left(\mathrm{CDCl}_{3}\right)$
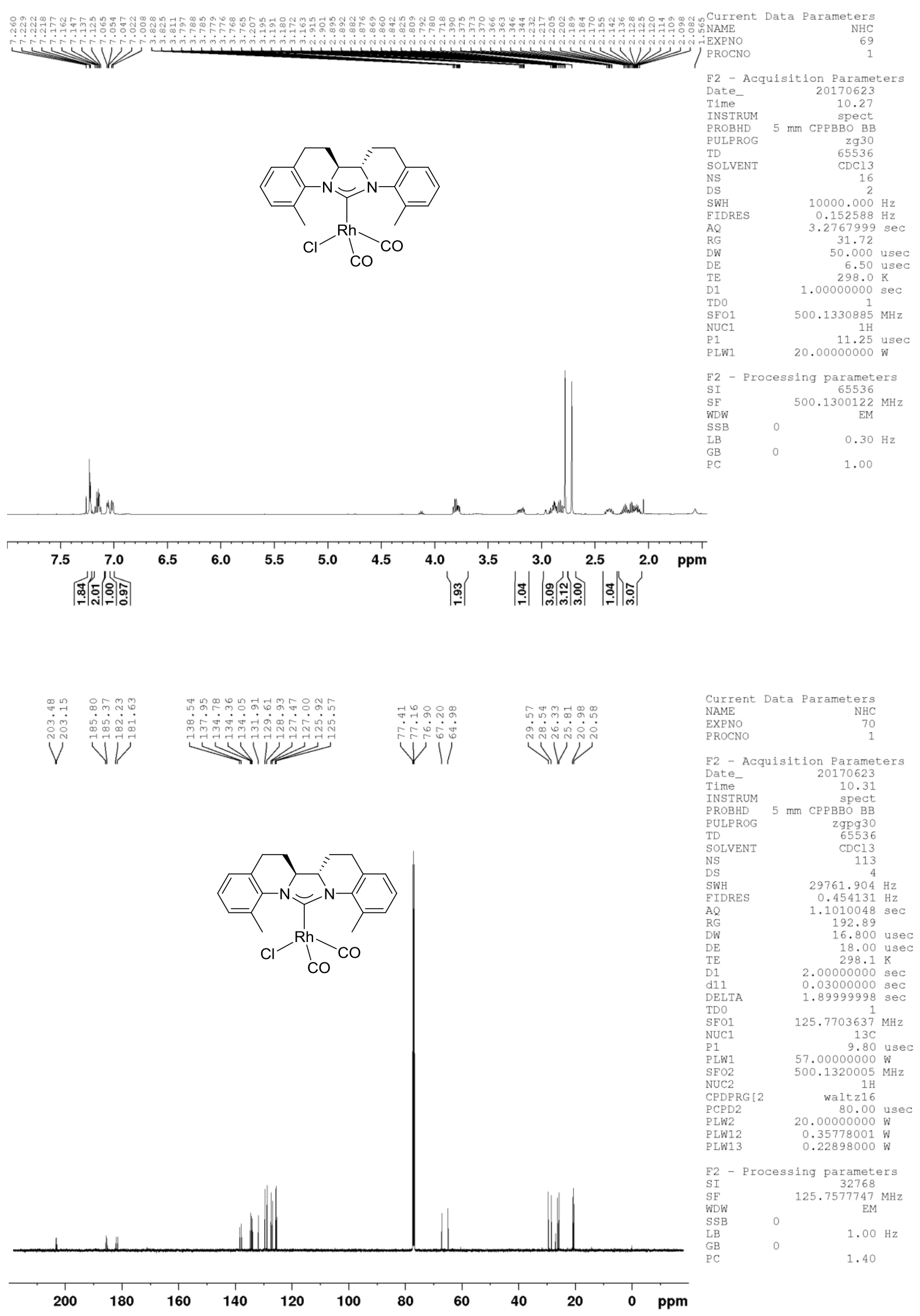
Figure S40. ${ }^{1} \mathrm{H}$ NMR and ${ }^{13} \mathrm{C}$ NMR spectra of $9 c\left(\mathrm{CDCl}_{3}\right)$
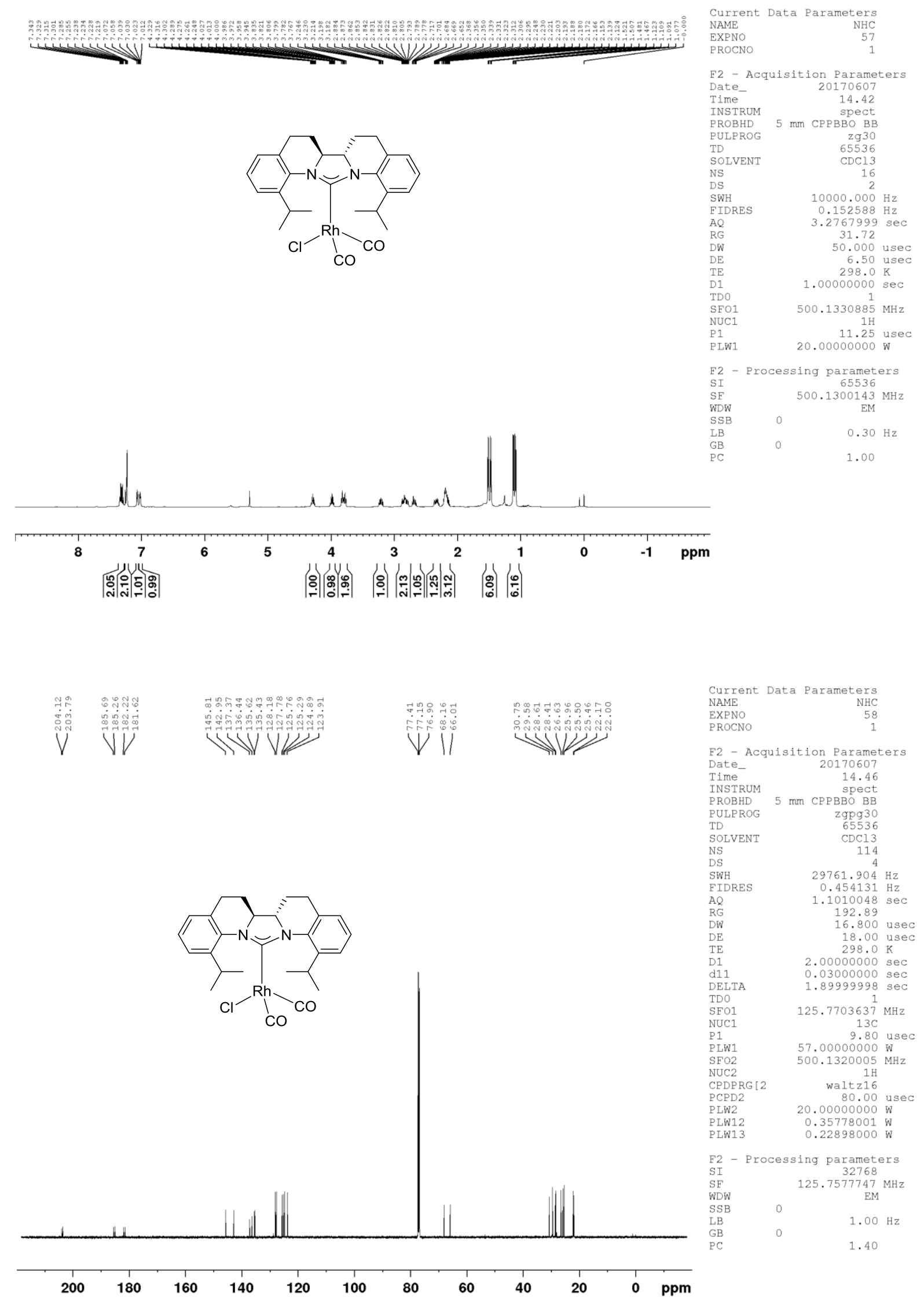
Figure $\mathbf{S 4 1}{ }^{1} \mathrm{H} \mathrm{NMR}$ and ${ }^{13} \mathrm{C}$ NMR spectra of $9 d\left(\mathrm{CDCl}_{3}\right)$

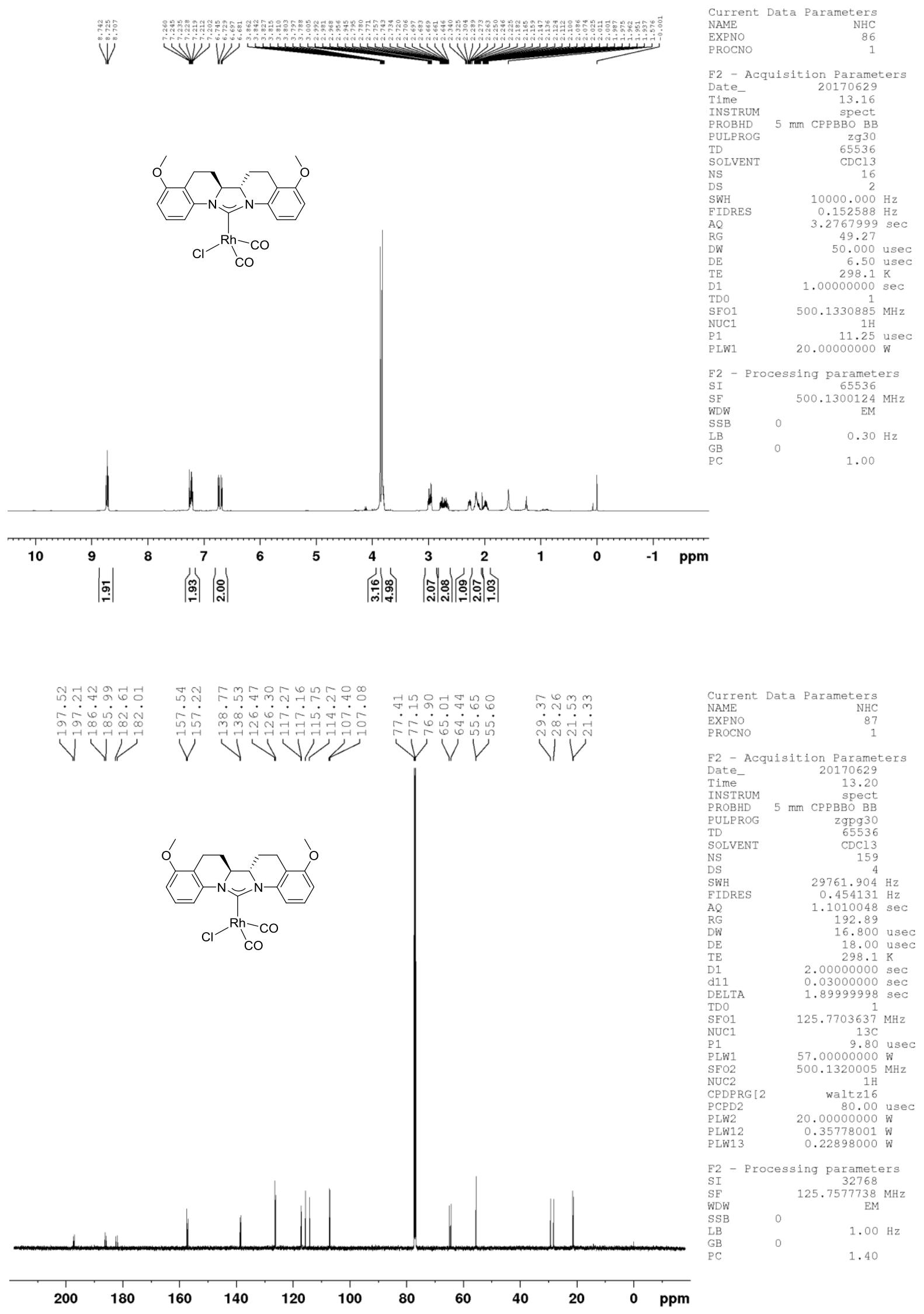


Figure S42. ${ }^{1} \mathrm{H}$ NMR and ${ }^{13} \mathrm{C}$ NMR spectra of $9 e\left(\mathrm{CDCl}_{3}\right)$

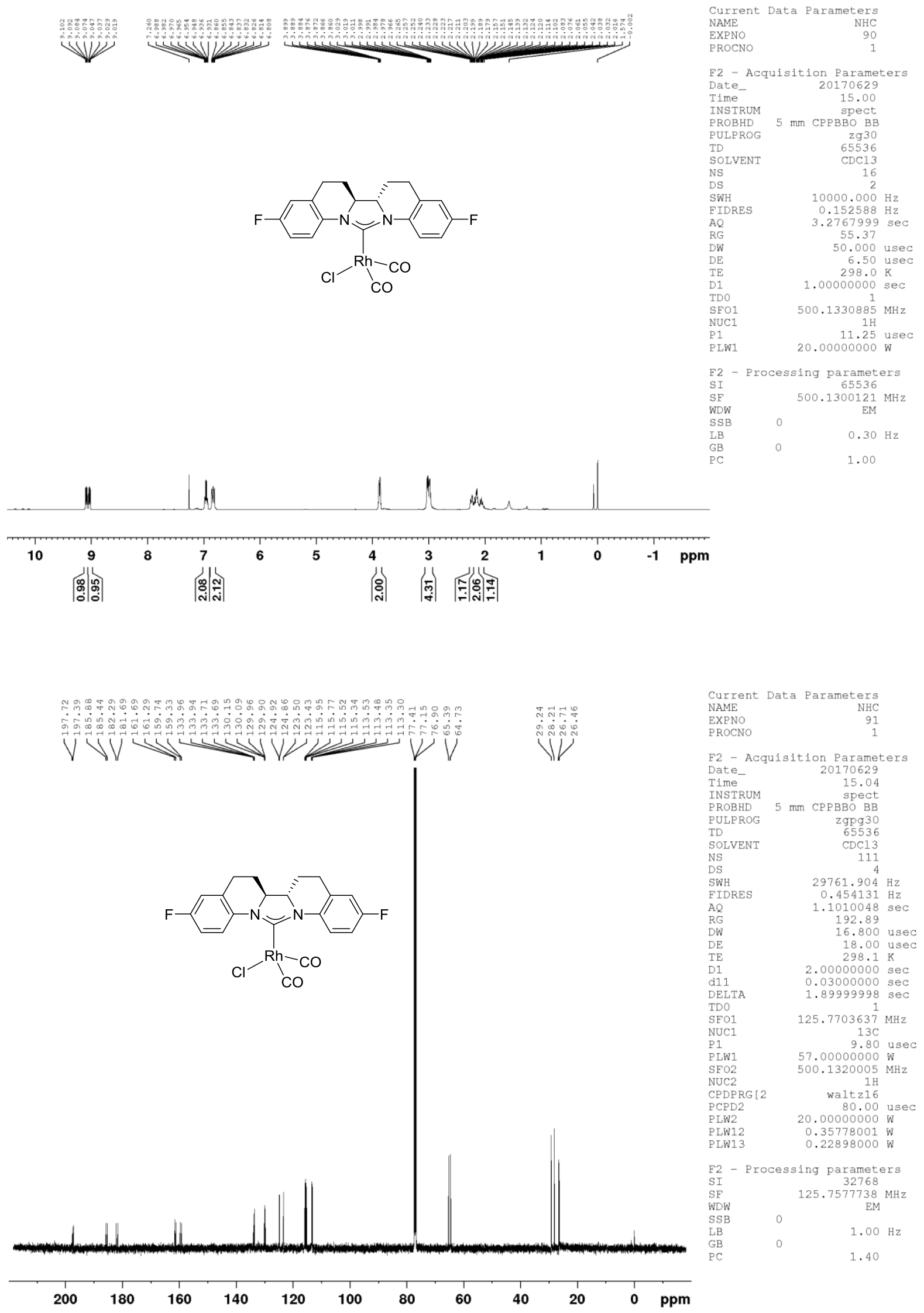


Figure S43. ${ }^{1} \mathrm{H}$ NMR and ${ }^{13} \mathrm{C}$ NMR spectra of $9 f\left(\mathrm{CDCl}_{3}\right)$
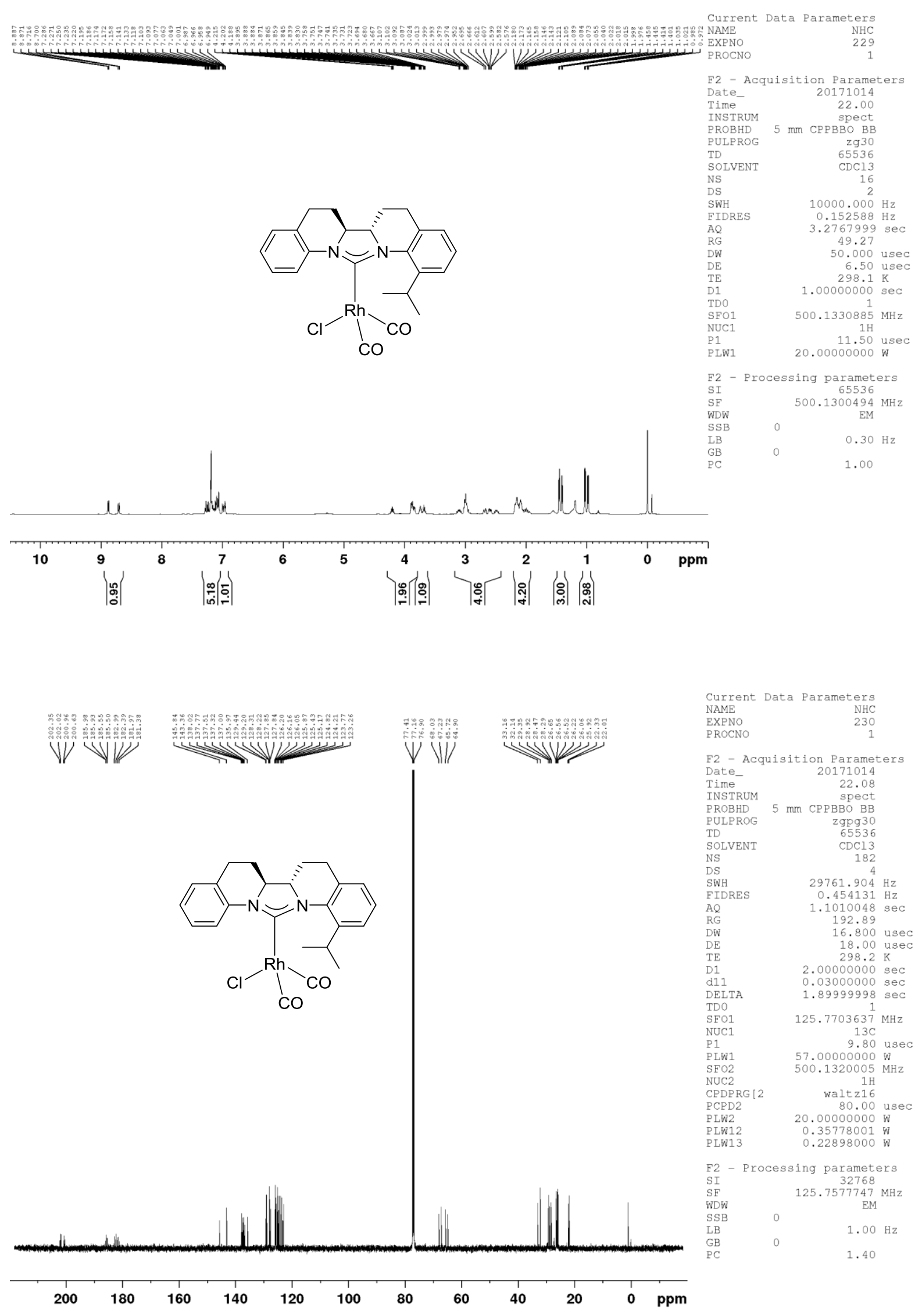
Figure S44. ${ }^{1} \mathrm{H}$ NMR and ${ }^{13} \mathrm{C}$ NMR spectra of $9 \mathrm{~g}\left(\mathrm{CDCl}_{3}\right)$

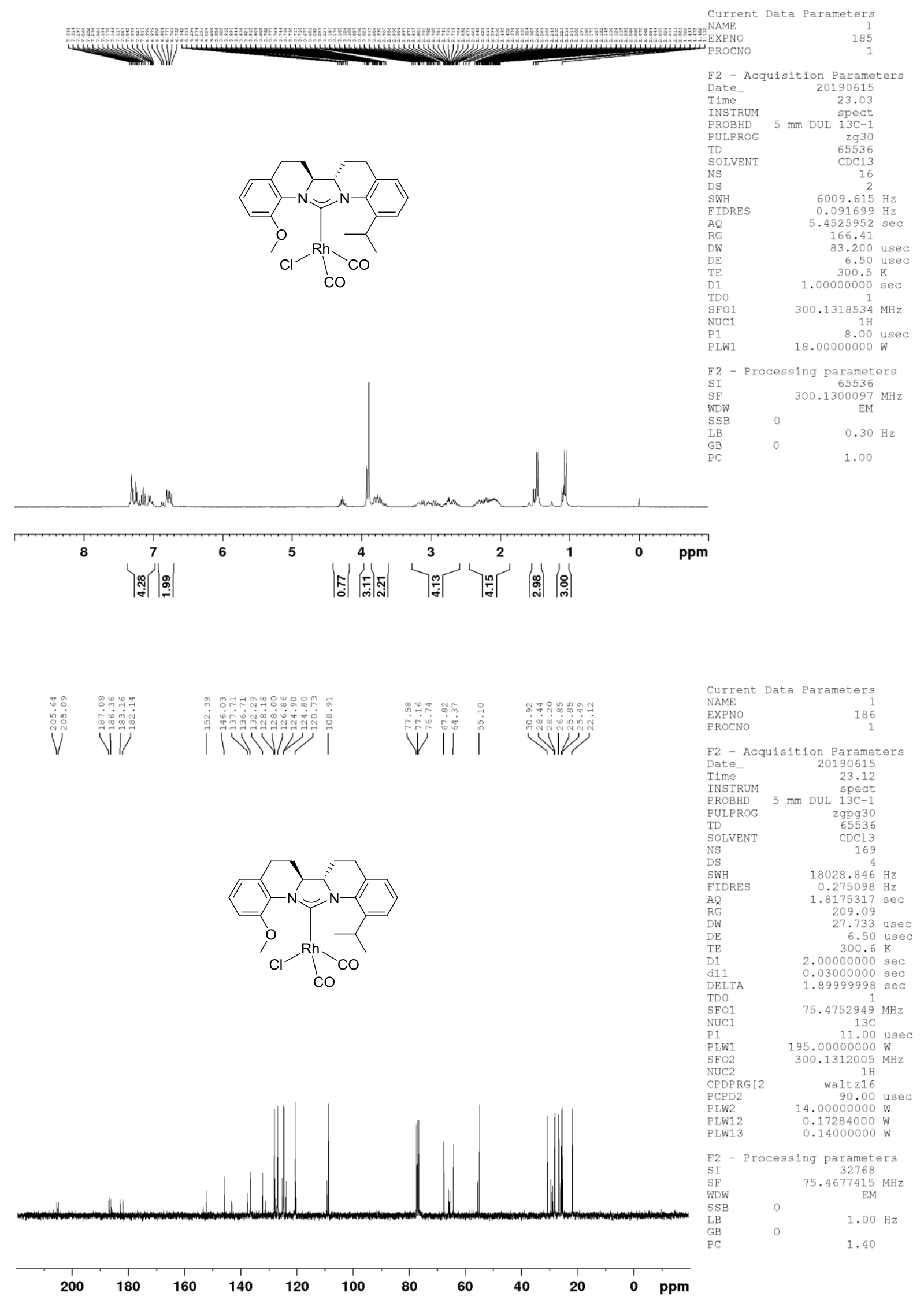


Figure S45. ${ }^{1} \mathrm{H} \mathrm{NMR}$ and ${ }^{13} \mathrm{C}$ NMR spectra of $11 \mathrm{a}\left(\mathrm{CDCl}_{3}\right)$
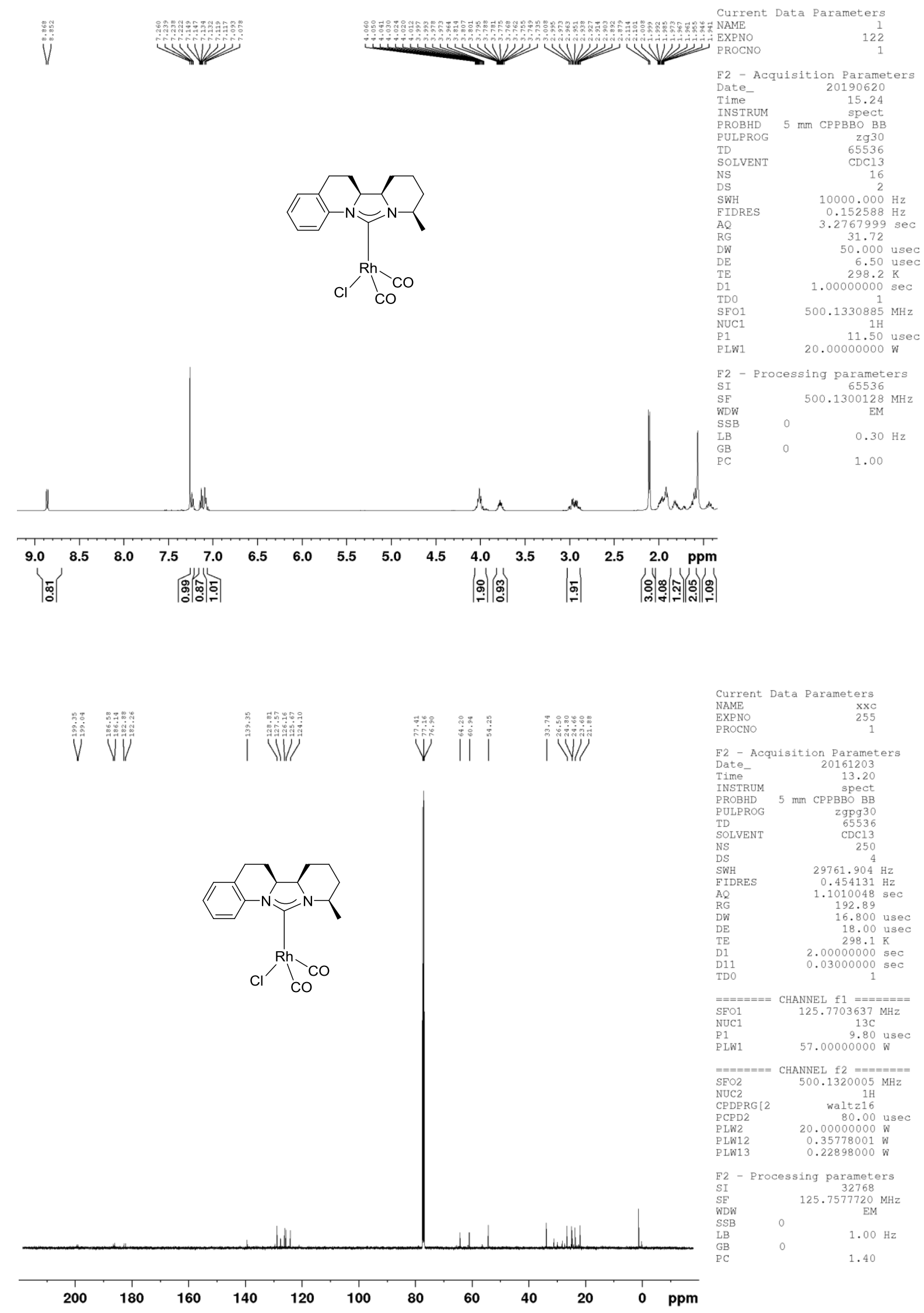
Figure S46. ${ }^{1} \mathrm{H} \mathrm{NMR}$ and ${ }^{13} \mathrm{C} \mathrm{NMR}$ spectra of $\mathbf{1 1 b}\left(\mathrm{CDCl}_{3}\right)$
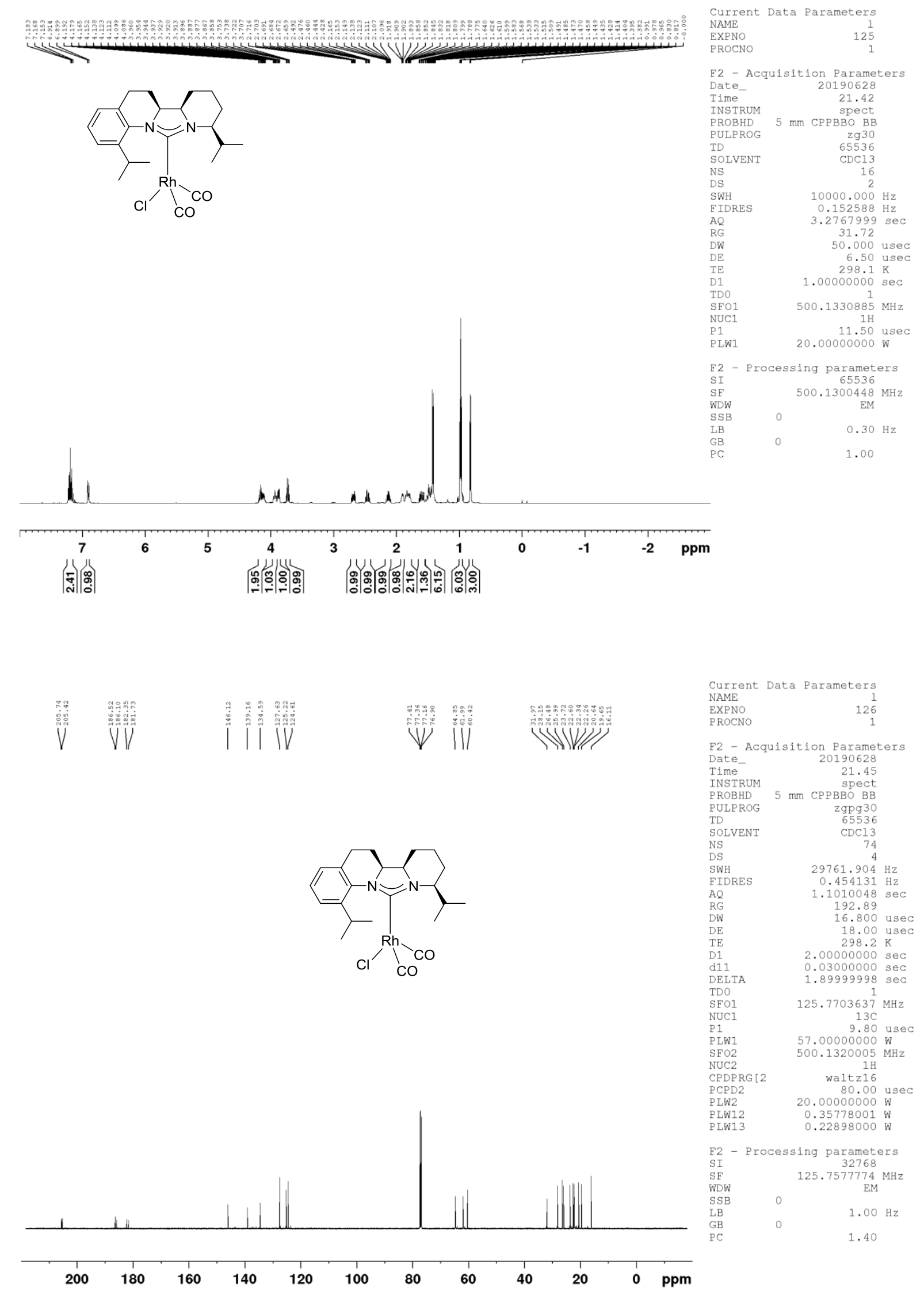
Figure S47. ${ }^{1} \mathrm{H}$ NMR and ${ }^{13} \mathrm{C}$ NMR spectra of $11 \mathrm{c}\left(\mathrm{CDCl}_{3}\right)$

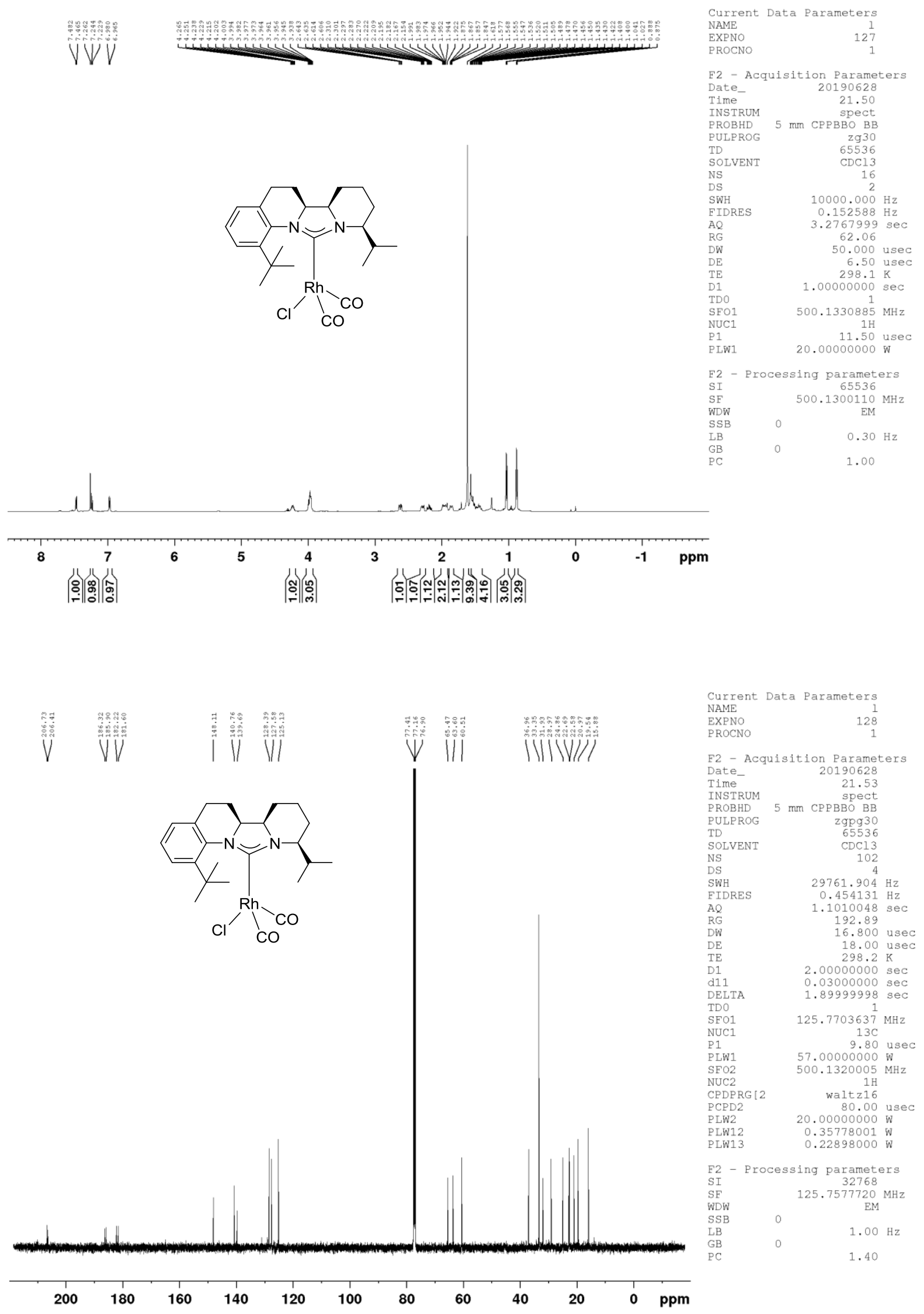


Figure S48. ${ }^{1} \mathrm{H}$ NMR and ${ }^{13} \mathrm{C}$ NMR spectra of $11 d\left(\mathrm{CDCl}_{3}\right)$
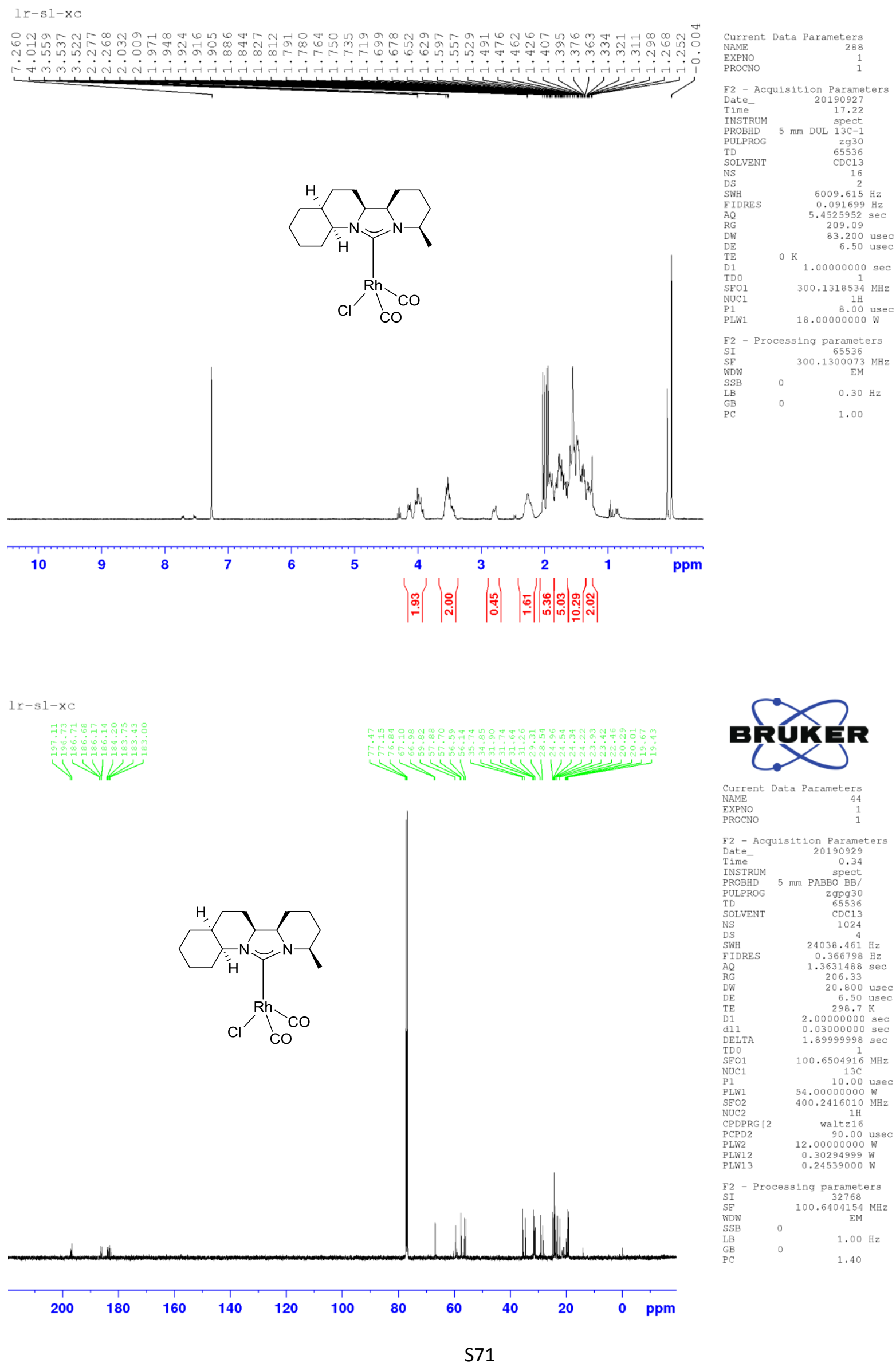
Figure S49. ${ }^{1} \mathrm{H}$ NMR and ${ }^{13} \mathrm{C}$ NMR spectra of $12\left(\mathrm{CDCl}_{3}\right)$
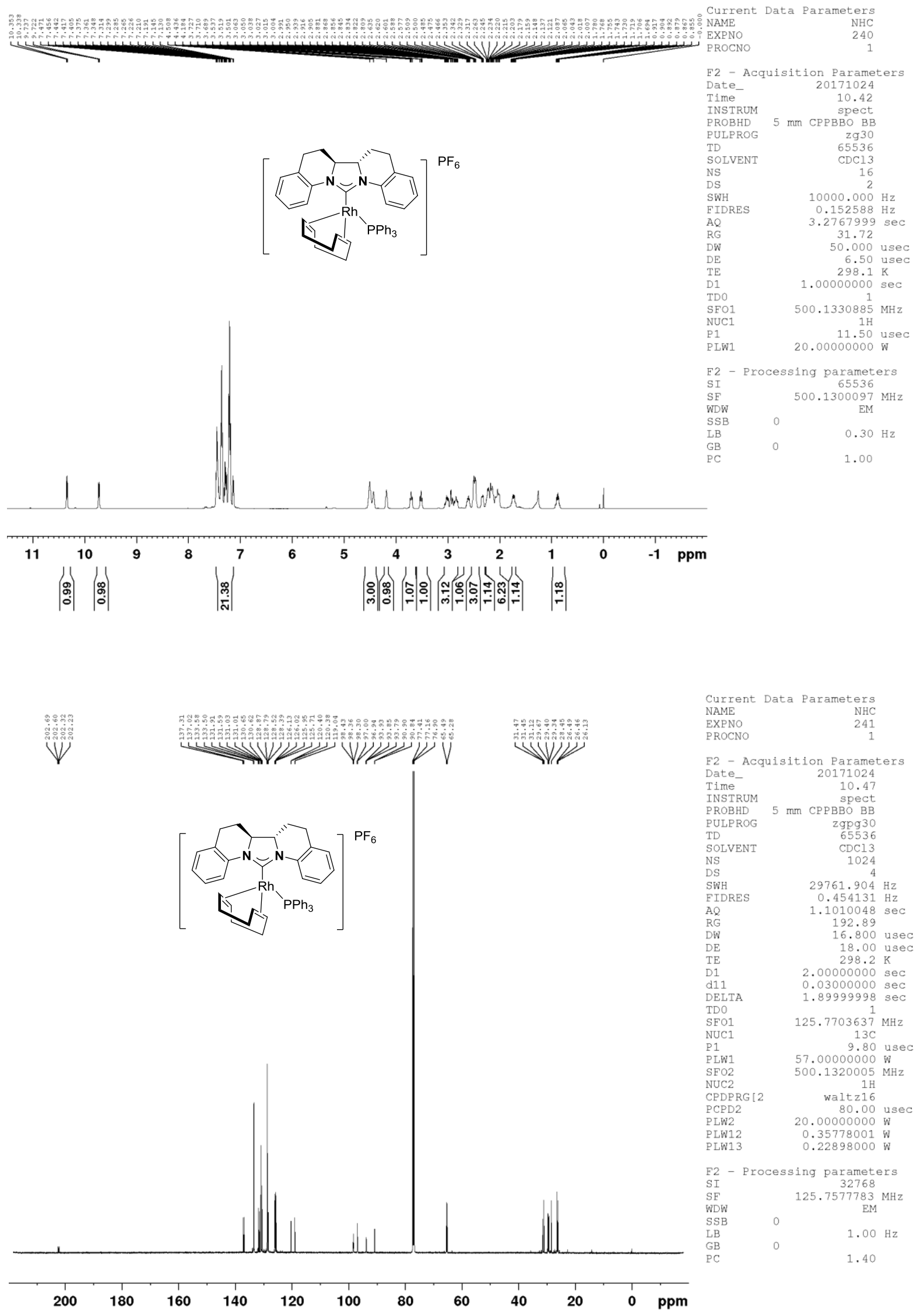


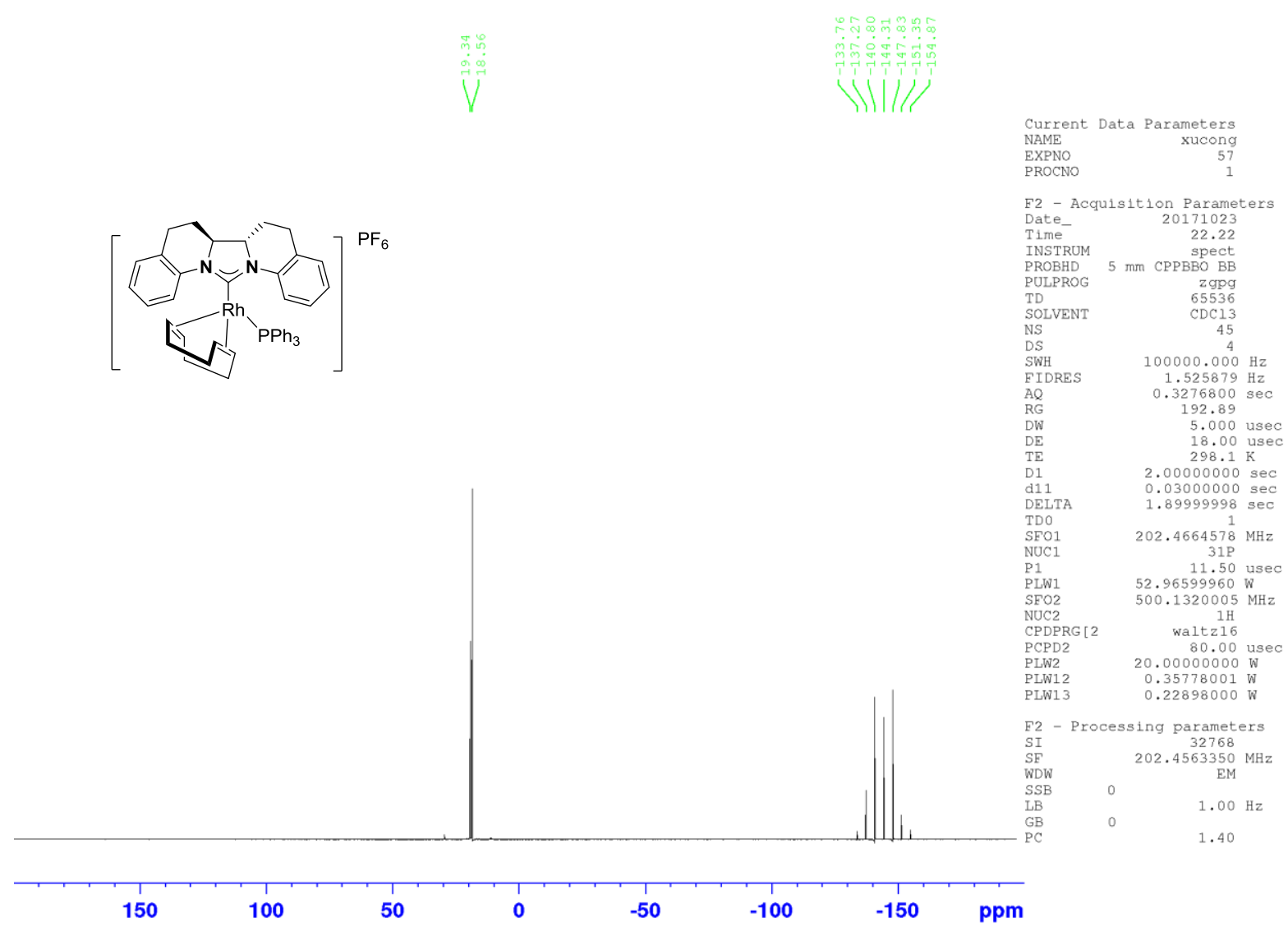

Figure S50. ${ }^{1} \mathrm{H}$ NMR and ${ }^{13} \mathrm{C}$ NMR spectra of $\mathbf{1 3}\left(\mathrm{CDCl}_{3}\right)$

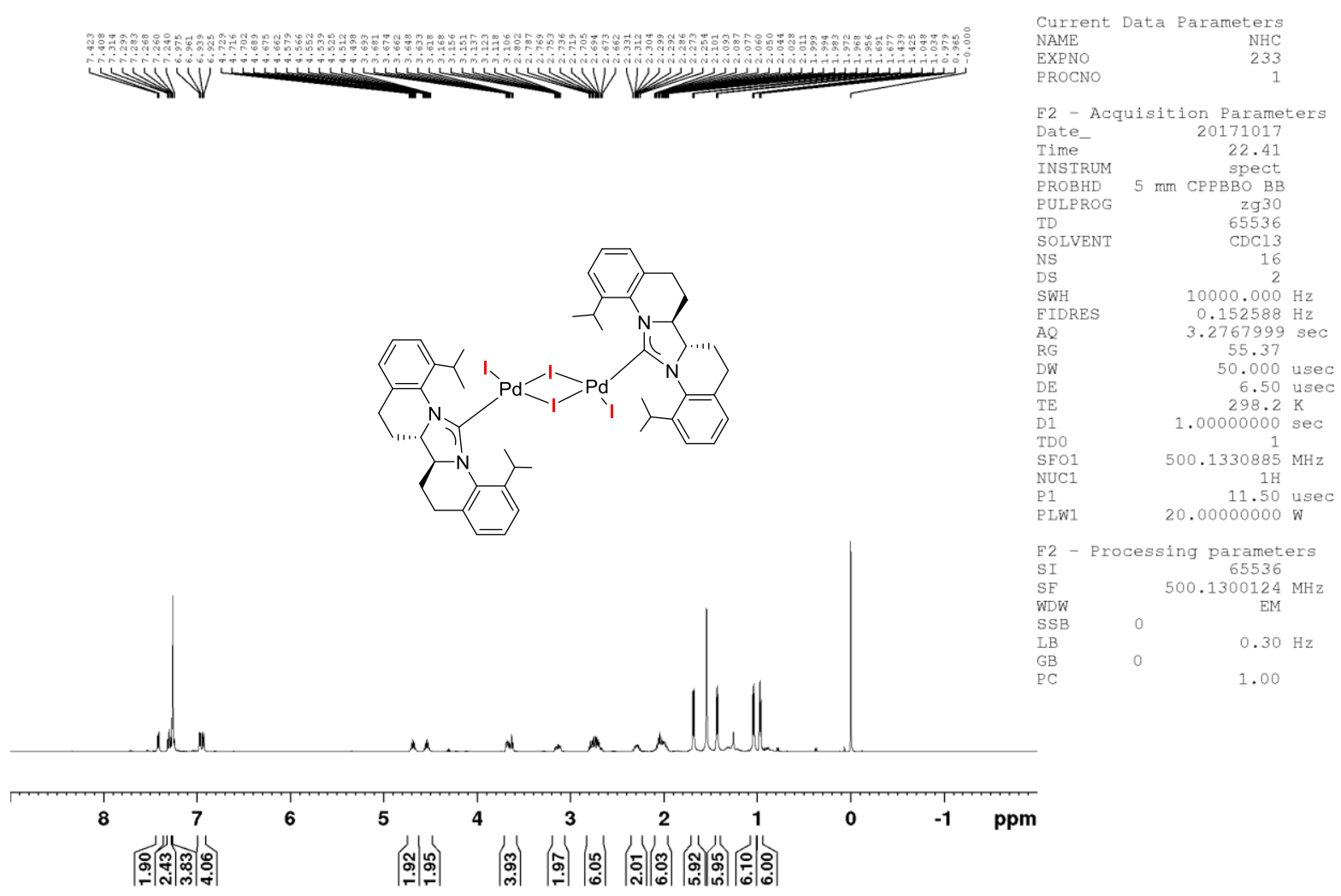




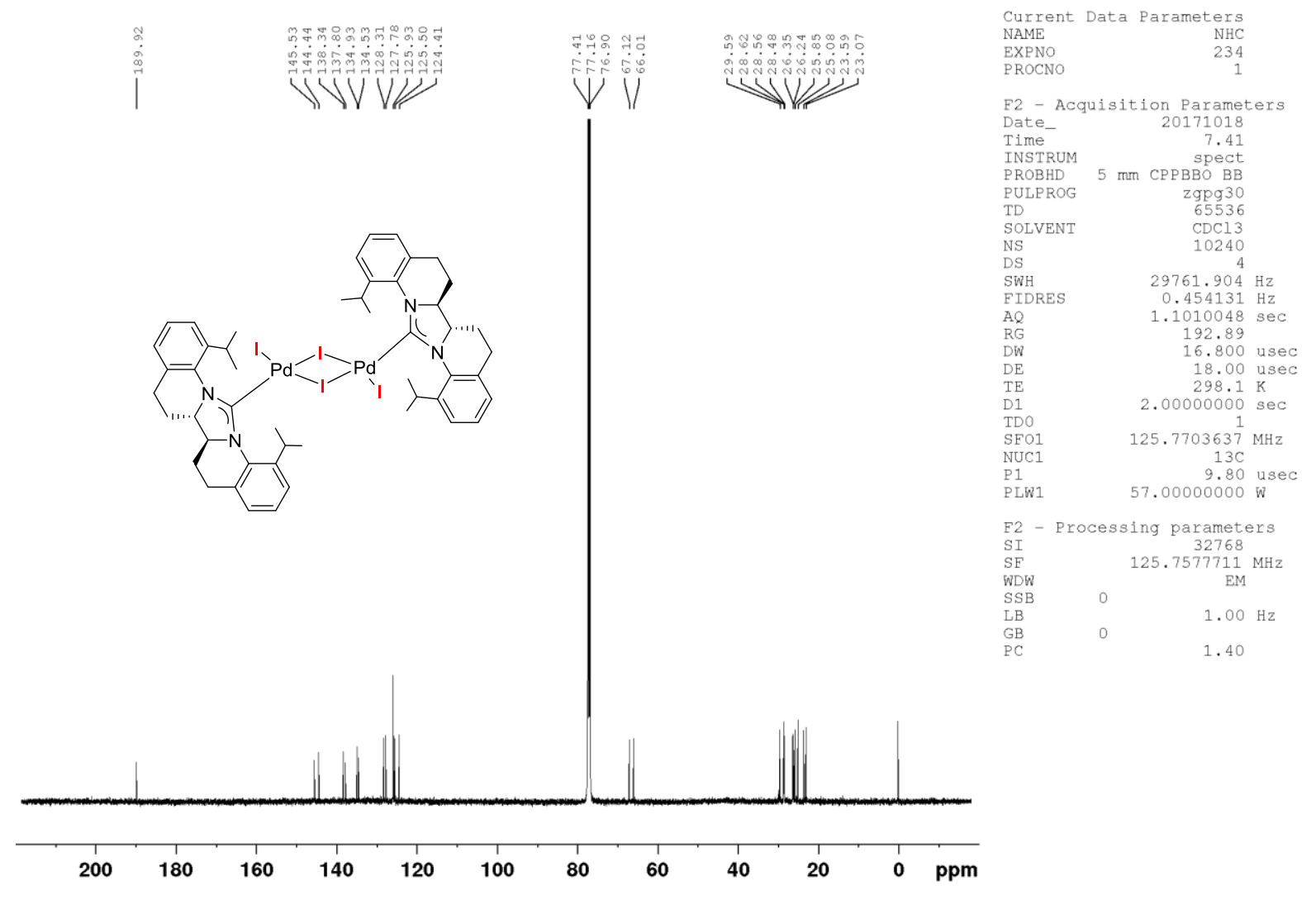


Figure S51. HPLC spectra of compounds 2a

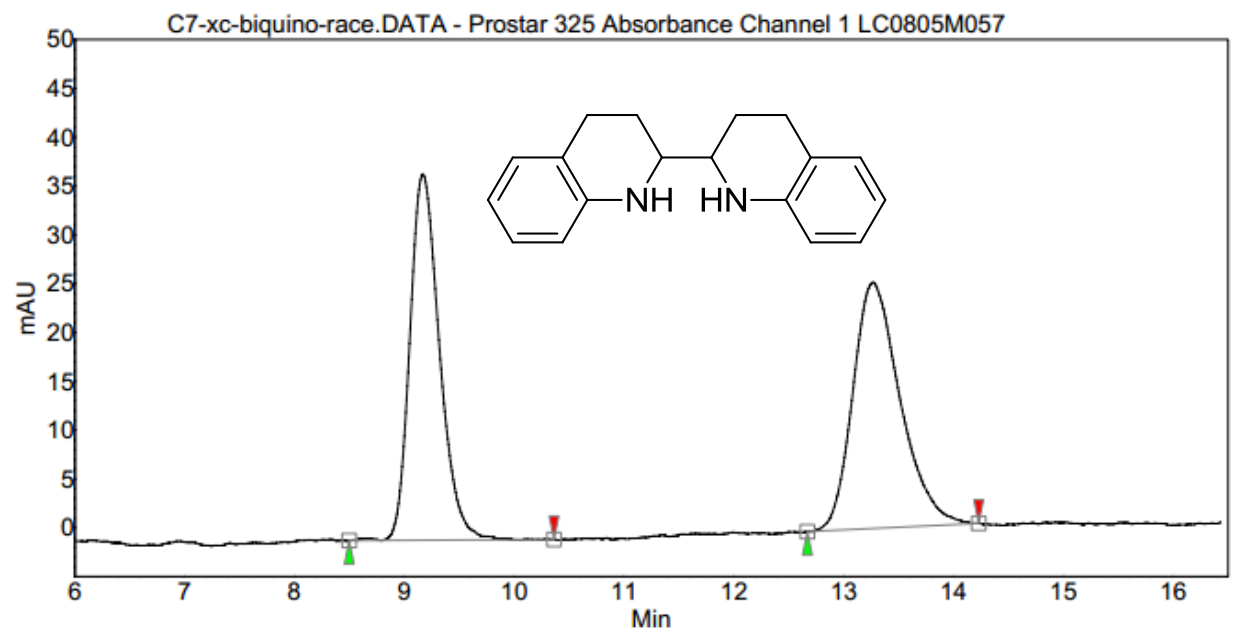

Peak results :

\begin{tabular}{|c|l|r|r|r|r|r|}
\hline Index & 文件名 & $\begin{array}{r}\text { 时间 } \\
\text { [Min] }\end{array}$ & $\begin{array}{r}\text { 数量 } \\
\text { [\% 面积] }\end{array}$ & $\begin{array}{r}\text { 高度 } \\
\text { [mAU] }\end{array}$ & $\begin{array}{r}\text { Area } \\
\text { [mAU.Min] }\end{array}$ & $\begin{array}{r}\text { Area \% } \\
{[\%]}\end{array}$ \\
\hline \hline 1 & 未知 & 9.17 & 48.28 & 37.5 & 11.7 & 48.281 \\
\hline 2 & 未知 & 13.27 & 51.72 & 25.2 & 12.6 & 51.719 \\
\hline & & & & & & \\
\hline Total & & & 100.00 & 62.7 & 24.3 & 100.000 \\
\hline
\end{tabular}

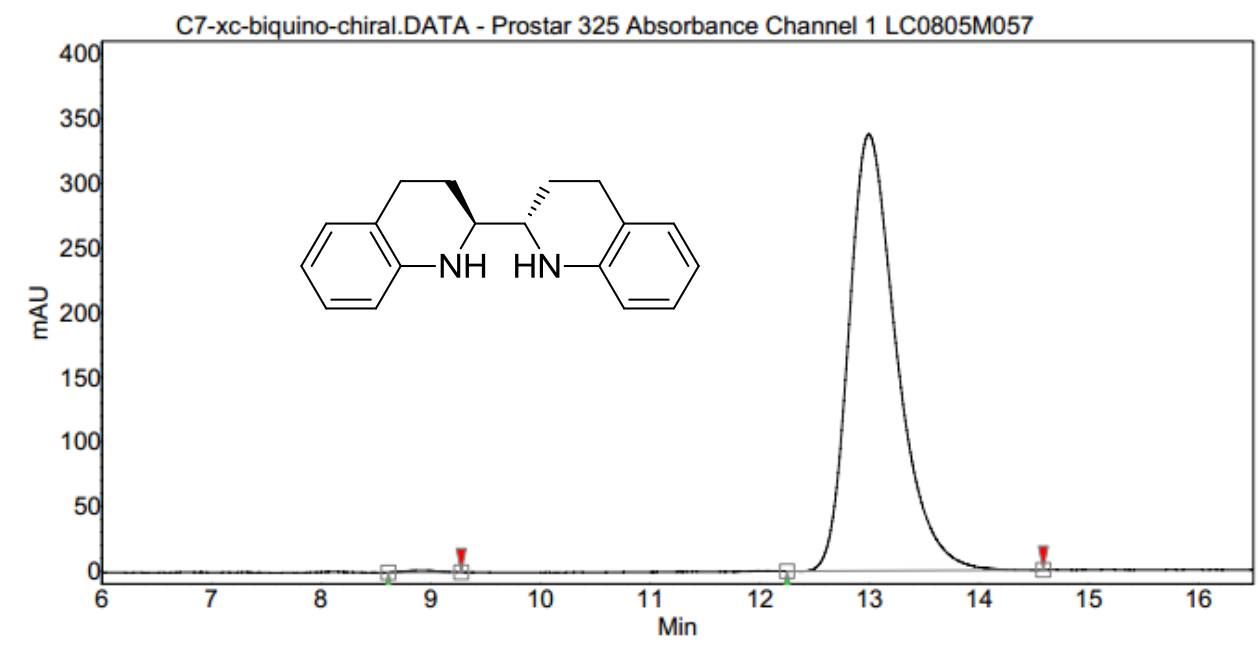

Peak results :

\begin{tabular}{|c|l|r|r|r|r|r|}
\hline Index & 文件名 & $\begin{array}{r}\text { 时间] } \\
\text { [Min] }\end{array}$ & $\begin{array}{r}\text { 数量 } \\
\text { [\% 面积] }\end{array}$ & $\begin{array}{r}\text { 高度 } \\
\text { [mAU] }\end{array}$ & $\begin{array}{r}\text { Area } \\
\text { [mAU.Min] }\end{array}$ & $\begin{array}{r}\text { Area \% } \\
{[\%]}\end{array}$ \\
\hline \hline 2 & 未知 & 8.93 & 0.30 & 1.5 & 0.5 & 0.301 \\
\hline 1 & 未知 & 12.99 & 99.70 & 337.8 & 170.0 & 99.699 \\
\hline & & & & & & \\
\hline Total & & & 100.00 & 339.3 & 170.5 & 100.000 \\
\hline
\end{tabular}


Figure S52. HPLC spectra of compounds 2b

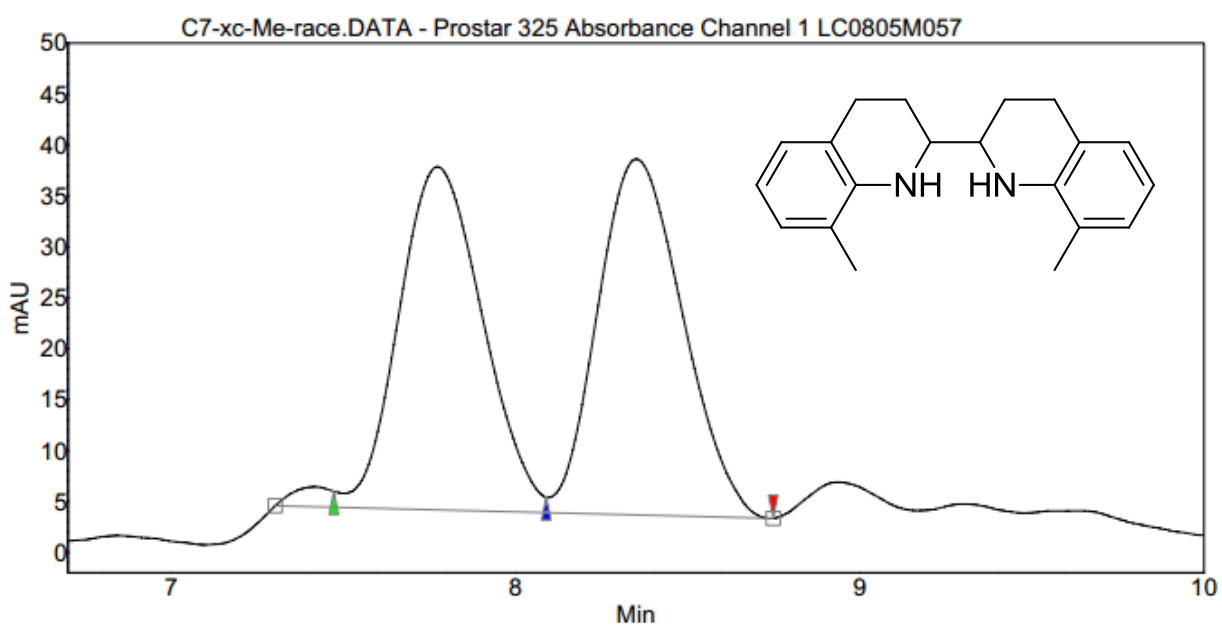

Peak results :

\begin{tabular}{|c|l|r|r|r|r|r|}
\hline Index & 文件名 & $\begin{array}{r}\text { 时间 } \\
\text { [Min] }\end{array}$ & $\begin{array}{r}\text { 数量 } \\
\text { [\% 面积 }\end{array}$ & $\begin{array}{r}\text { 高度 } \\
\text { [mAU] }\end{array}$ & $\begin{array}{r}\text { Area } \\
\text { [mAU.Min] }\end{array}$ & $\begin{array}{r}\text { Area \% } \\
\text { [\%] }\end{array}$ \\
\hline \hline 2 & 未知 & 7.77 & 48.77 & 33.7 & 9.5 & 48.769 \\
\hline 1 & 未知 & 8.35 & 51.23 & 34.9 & 10.0 & 51.231 \\
\hline & & & & & & \\
\hline Total & & & 100.00 & 68.6 & 19.5 & 100.000 \\
\hline
\end{tabular}

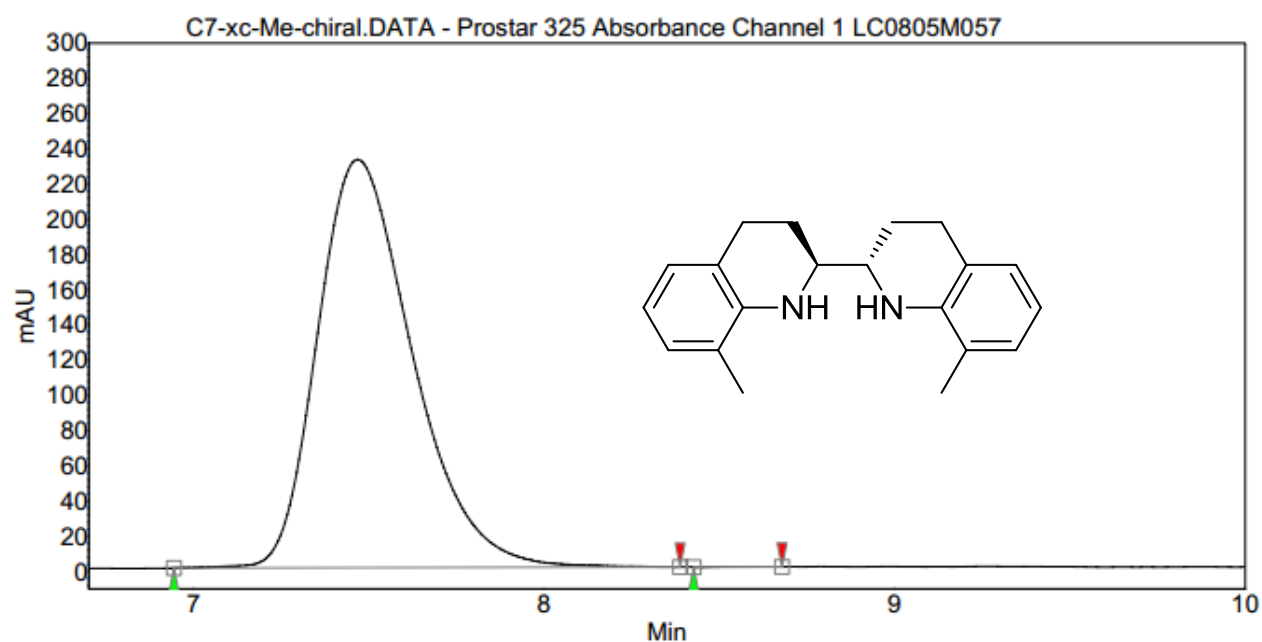

Peak results :

\begin{tabular}{|c|l|r|r|r|r|r|}
\hline Index & 文件名 & $\begin{array}{r}\text { 时间 } \\
\text { [Min] }\end{array}$ & $\begin{array}{r}\text { 数量 } \\
\text { [\% 面积 }\end{array}$ & $\begin{array}{r}\text { 高度 } \\
\text { [mAU] }\end{array}$ & $\begin{array}{r}\text { Area } \\
\text { [mAU.Min] }\end{array}$ & $\begin{array}{r}\text { Area \% } \\
\text { [\%] }\end{array}$ \\
\hline \hline 1 & 未觙 & 7.47 & 99.97 & 231.8 & 72.9 & 99.974 \\
\hline 2 & 未知 & 8.52 & 0.03 & 0.2 & 0.0 & 0.026 \\
\hline & & & & & & \\
\hline Total & & & 100.00 & 232.0 & 72.9 & 100.000 \\
\hline
\end{tabular}


Figure S53. HPLC spectra of compounds 2c

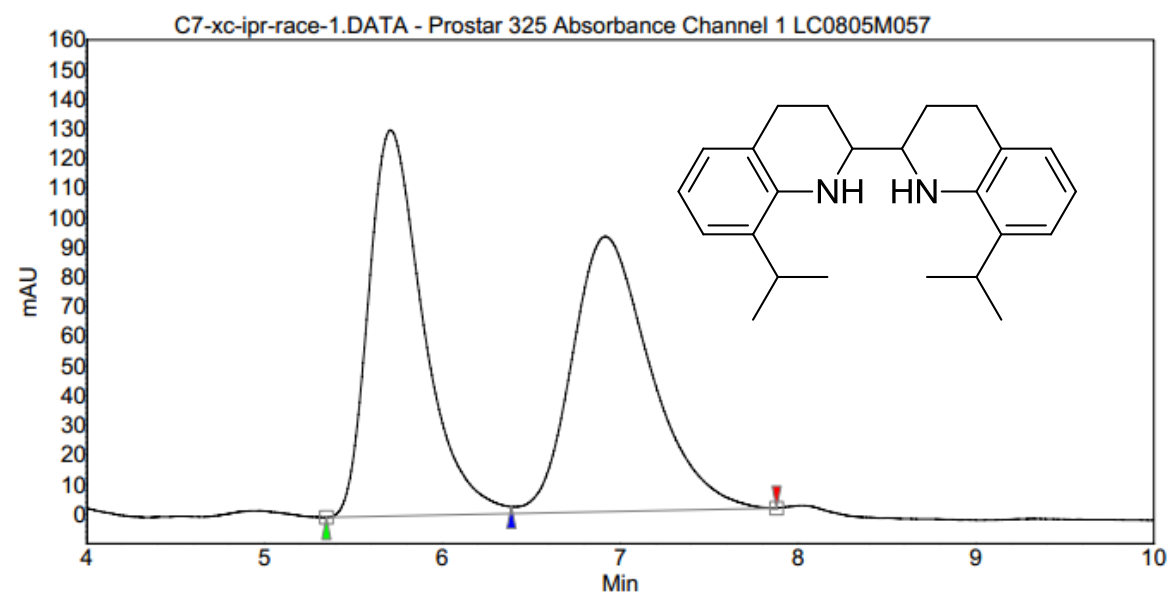

Peak results :

\begin{tabular}{|c|l|r|r|r|r|r|}
\hline Index & 文件名 & $\begin{array}{r}\text { 时间 } \\
\text { [Min] }\end{array}$ & $\begin{array}{r}\text { 数量 } \\
\text { [\% 面积] }\end{array}$ & $\begin{array}{r}\text { 高度 } \\
\text { [mAU] }\end{array}$ & $\begin{array}{r}\text { Area } \\
\text { [mAU.Min] }\end{array}$ & $\begin{array}{r}\text { Area \% } \\
\text { [\%] }\end{array}$ \\
\hline \hline 1 & 未知 & 5.71 & 49.37 & 130.2 & 46.4 & 49.366 \\
\hline 2 & 未知 & 6.91 & 50.63 & 92.8 & 47.6 & 50.634 \\
\hline & & & & & & \\
\hline Total & & & 100.00 & 223.1 & 93.9 & 100.000 \\
\hline
\end{tabular}

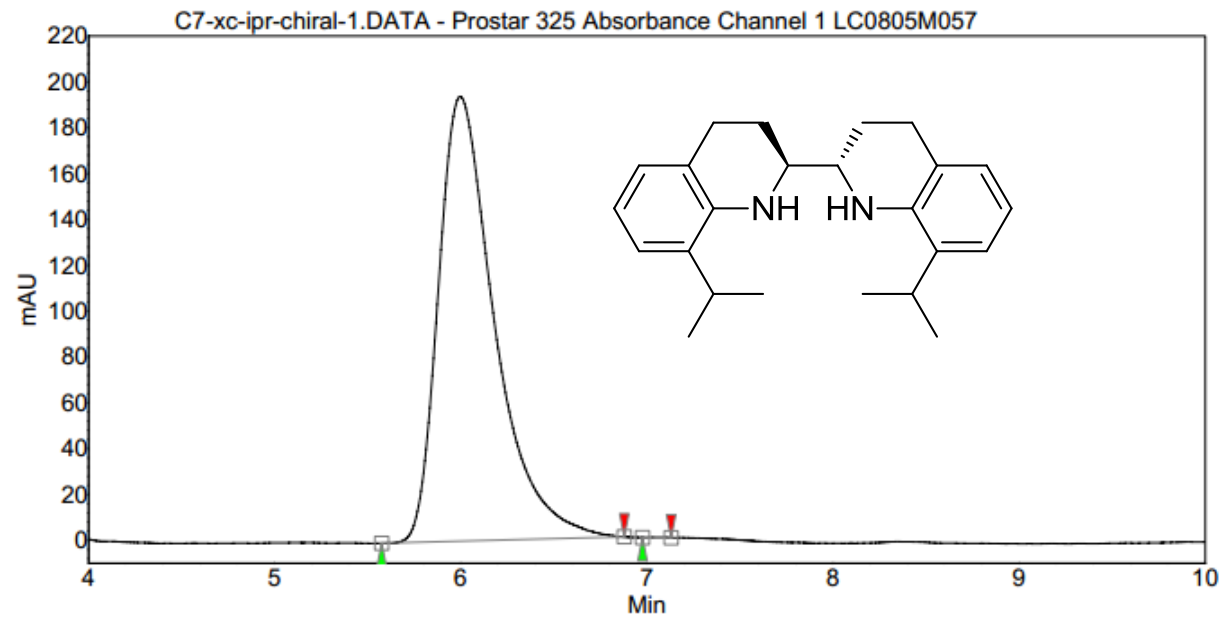

Peak results :

\begin{tabular}{|c|l|r|r|r|r|r|}
\hline Index & 文件名 & $\begin{array}{r}\text { 时间 } \\
\text { [Min] }\end{array}$ & $\begin{array}{r}\text { 数量 } \\
\text { [\% 面积] }\end{array}$ & $\begin{array}{r}\text { 高度 } \\
\text { [mAU] }\end{array}$ & $\begin{array}{r}\text { Area } \\
\text { [mAU.Min] }\end{array}$ & $\begin{array}{r}\text { Area \% } \\
{[\%]}\end{array}$ \\
\hline \hline 1 & 未每] & 5.99 & 99.99 & 193.9 & 67.7 & 99.992 \\
\hline 2 & 未知 & 7.09 & 0.01 & 0.1 & 0.0 & 0.008 \\
\hline & & & & & & \\
\hline Total & & & 100.00 & 194.0 & 67.7 & 100.000 \\
\hline
\end{tabular}


Figure S54. HPLC spectra of compounds 2d

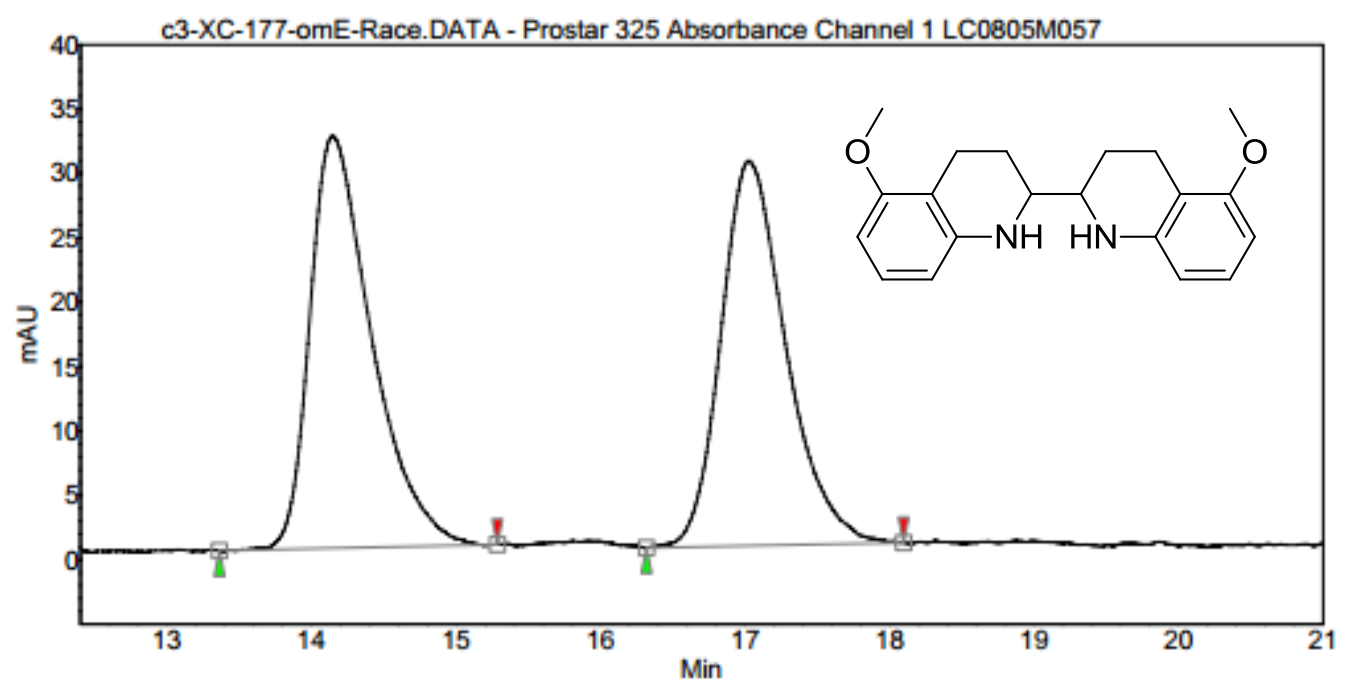

Peak results :

\begin{tabular}{|c|c|c|c|c|c|c|}
\hline Index & 文伊存 & $\begin{array}{l}\text { 时间 } \\
\text { Dinl }\end{array}$ & 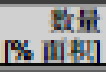 & $\begin{array}{r}\text { 高度 } \\
\text { ImAuI }\end{array}$ & $\begin{array}{r}\text { Area } \\
\text { [mAU.Min] }\end{array}$ & $\begin{array}{r}\text { Area } \% \\
{[\%]}\end{array}$ \\
\hline 1 & 本知 & 14.15 & 50.36 & 32.0 & 15.8 & 50.360 \\
\hline 2 & 本知 & 17.03 & 49.64 & 29.9 & 15.6 & 49.640 \\
\hline Total & & & 100.00 & 61.9 & 31.5 & 100.000 \\
\hline
\end{tabular}

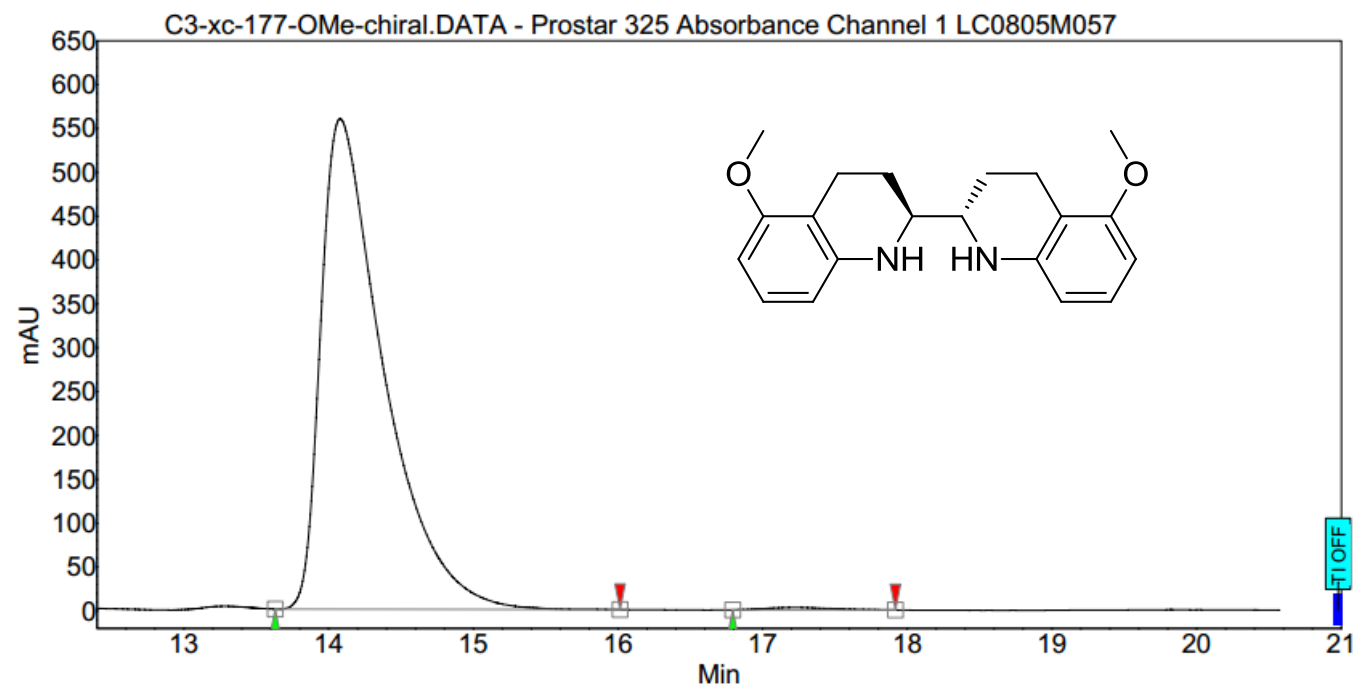

Peak results :

\begin{tabular}{|c|l|r|r|r|r|r|}
\hline Index & 文件名 & $\begin{array}{r}\text { 时间 } \\
\text { [Min] }\end{array}$ & $\begin{array}{r}\text { 数量 } \\
\text { \% 面积 }\end{array}$ & $\begin{array}{r}\text { 高度 } \\
\text { [mAU] }\end{array}$ & $\begin{array}{r}\text { Area } \\
\text { [mAU.Min] }\end{array}$ & $\begin{array}{r}\text { Area \% } \\
{[\%]}\end{array}$ \\
\hline \hline 1 & 未知 & 14.08 & 99.48 & 559.2 & 280.8 & 99.480 \\
\hline 2 & 未知 & 17.21 & 0.52 & 2.7 & 1.5 & 0.520 \\
\hline & & & & & & \\
\hline Total & & & 100.00 & 562.0 & 282.3 & 100.000 \\
\hline
\end{tabular}


Figure S55. HPLC spectra of compounds 2e

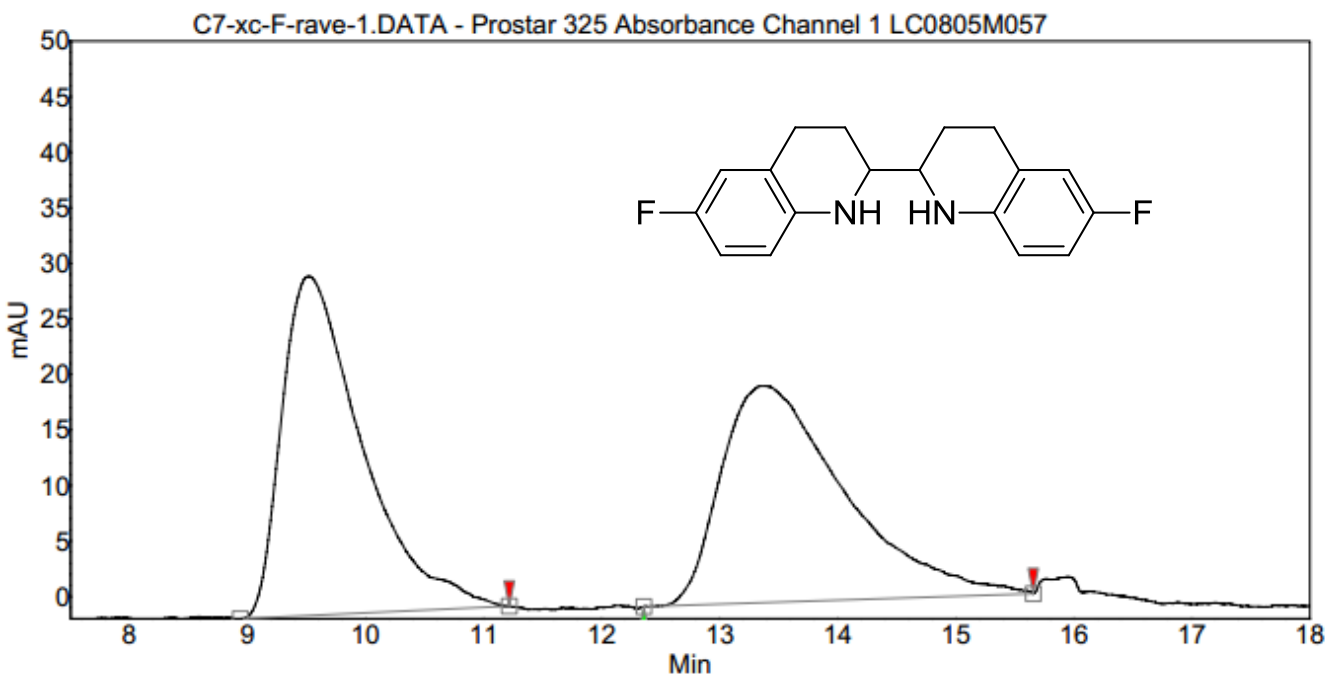

Peak results :

\begin{tabular}{|c|c|c|c|c|c|c|}
\hline Index & 文件名 & $\begin{array}{l}\text { 时间 } \\
\text { [Min] }\end{array}$ & $\begin{array}{r}\text { 数量 } \\
{[\% \text { 面积] }}\end{array}$ & $\begin{array}{l}\text { 高度 } \\
{[\mathrm{mAU}]}\end{array}$ & $\begin{array}{r}\text { Area } \\
\text { [mAU.Min] }\end{array}$ & $\begin{array}{r}\text { Area \% } \\
{[\%]}\end{array}$ \\
\hline 1 & 未知 & 9.52 & 50.48 & 30.5 & 24.1 & 50.482 \\
\hline 2 & 未知 & 13.35 & 49.52 & 19.5 & 23.6 & 49.518 \\
\hline Total & & & 100.00 & 50.0 & 47.7 & 100.000 \\
\hline
\end{tabular}

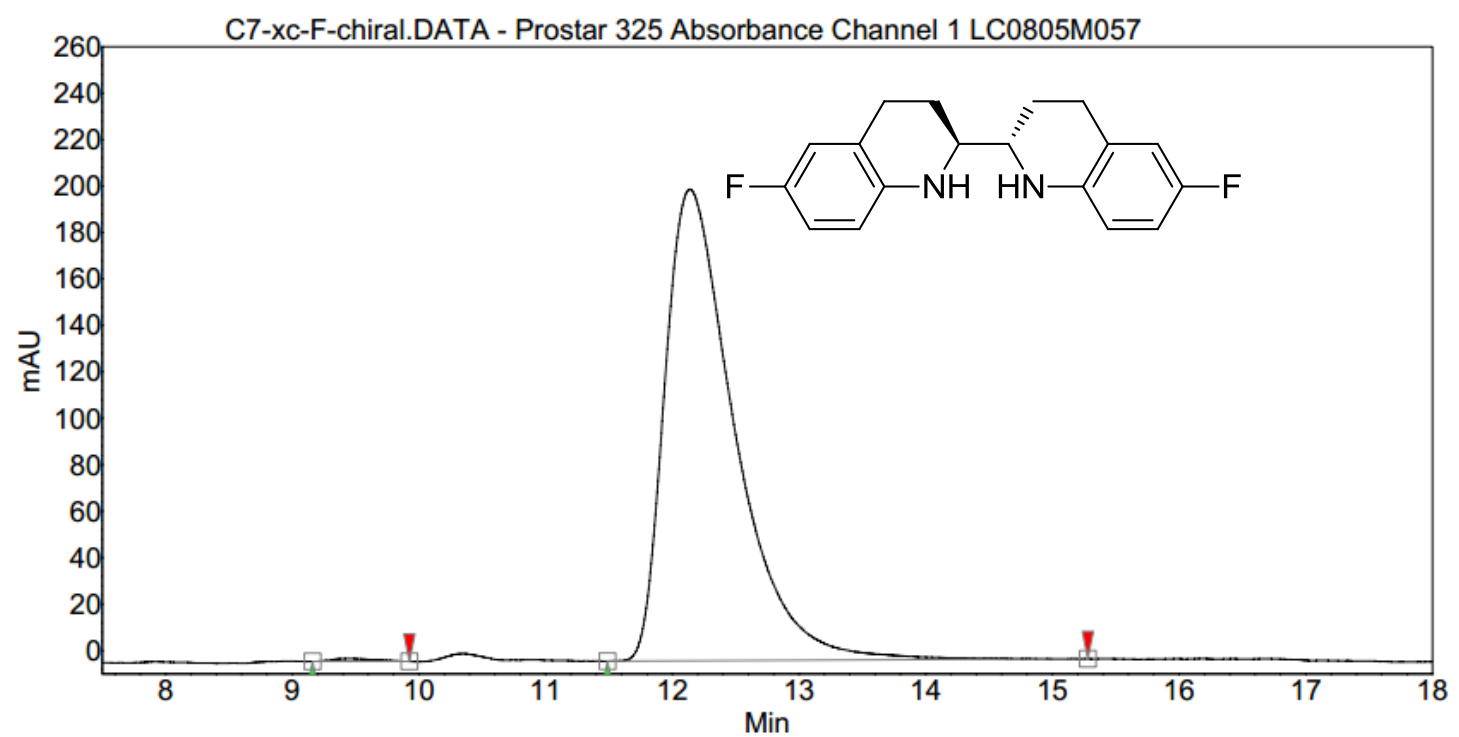

Peak results :

\begin{tabular}{|c|l|r|r|r|r|r|}
\hline Index & 文件名 & $\begin{array}{r}\text { 时间 } \\
\text { [Min] }\end{array}$ & $\begin{array}{r}\text { 数量 } \\
\text { [\% 面积] }\end{array}$ & $\begin{array}{r}\text { 高度 } \\
\text { [mAU] }\end{array}$ & $\begin{array}{r}\text { Area } \\
\text { [mAU.Min] }\end{array}$ & $\begin{array}{r}\text { Area \% } \\
\text { [\%] }\end{array}$ \\
\hline \hline 1 & 未知 & 9.46 & 0.35 & 1.2 & 0.5 & 0.354 \\
\hline 2 & 未知 & 12.14 & 99.65 & 203.0 & 129.9 & 99.646 \\
\hline & & & & & & \\
\hline Total & & & 100.00 & 204.2 & 130.4 & 100.000 \\
\hline
\end{tabular}


Figure S56. HPLC spectra of compounds $\mathbf{2 f}$

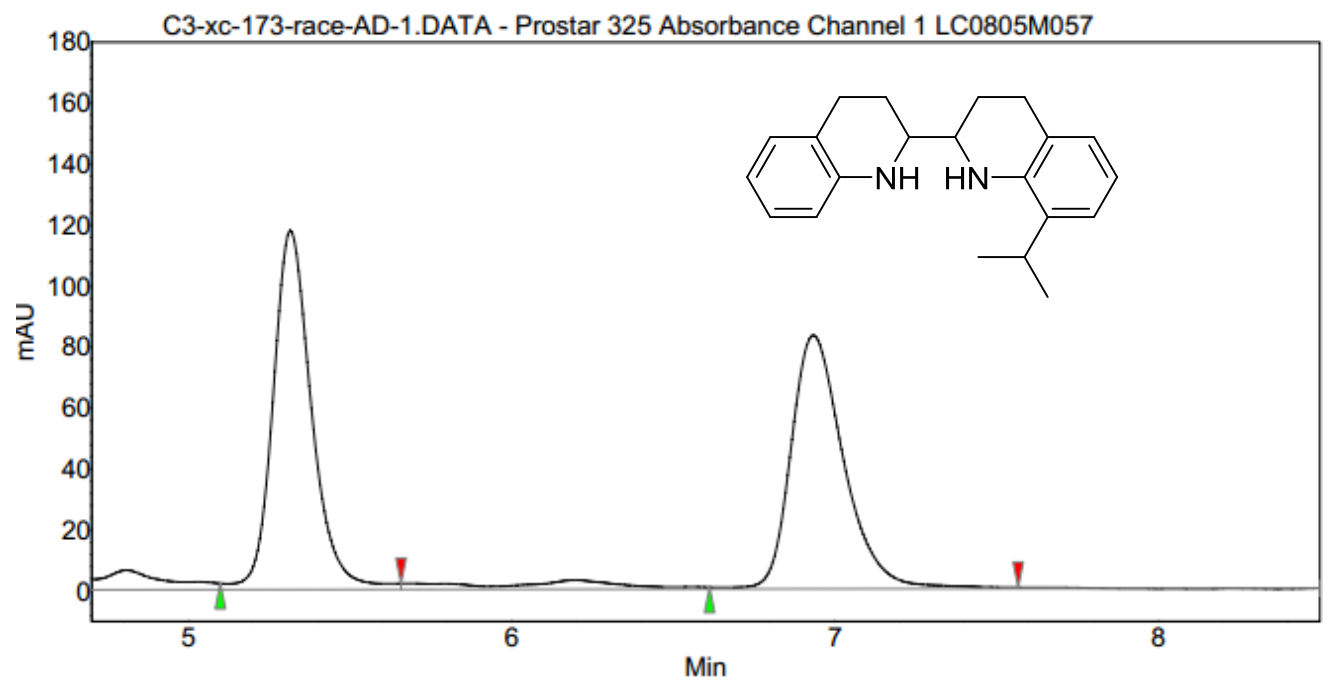

Peak results :

\begin{tabular}{|c|l|r|r|r|r|r|}
\hline Index & 文件名 & $\begin{array}{r}\text { 时间 } \\
{[\text { Min] }}\end{array}$ & $\begin{array}{r}\text { 数量 } \\
{[\% \text { 面积 }}\end{array}$ & $\begin{array}{r}\text { 高度 } \\
\text { [mAU] }\end{array}$ & $\begin{array}{r}\text { Area } \\
\text { [mAU.Min] }\end{array}$ & $\begin{array}{r}\text { Area \% } \\
\text { [\%] }\end{array}$ \\
\hline \hline 1 & 未知 & 5.31 & 50.92 & 117.8 & 16.4 & 50.916 \\
\hline 2 & 未知 & 6.93 & 49.08 & 83.2 & 15.8 & 49.084 \\
\hline & & & & & & \\
\hline Total & & & 100.00 & 201.0 & 32.2 & 100.000 \\
\hline
\end{tabular}

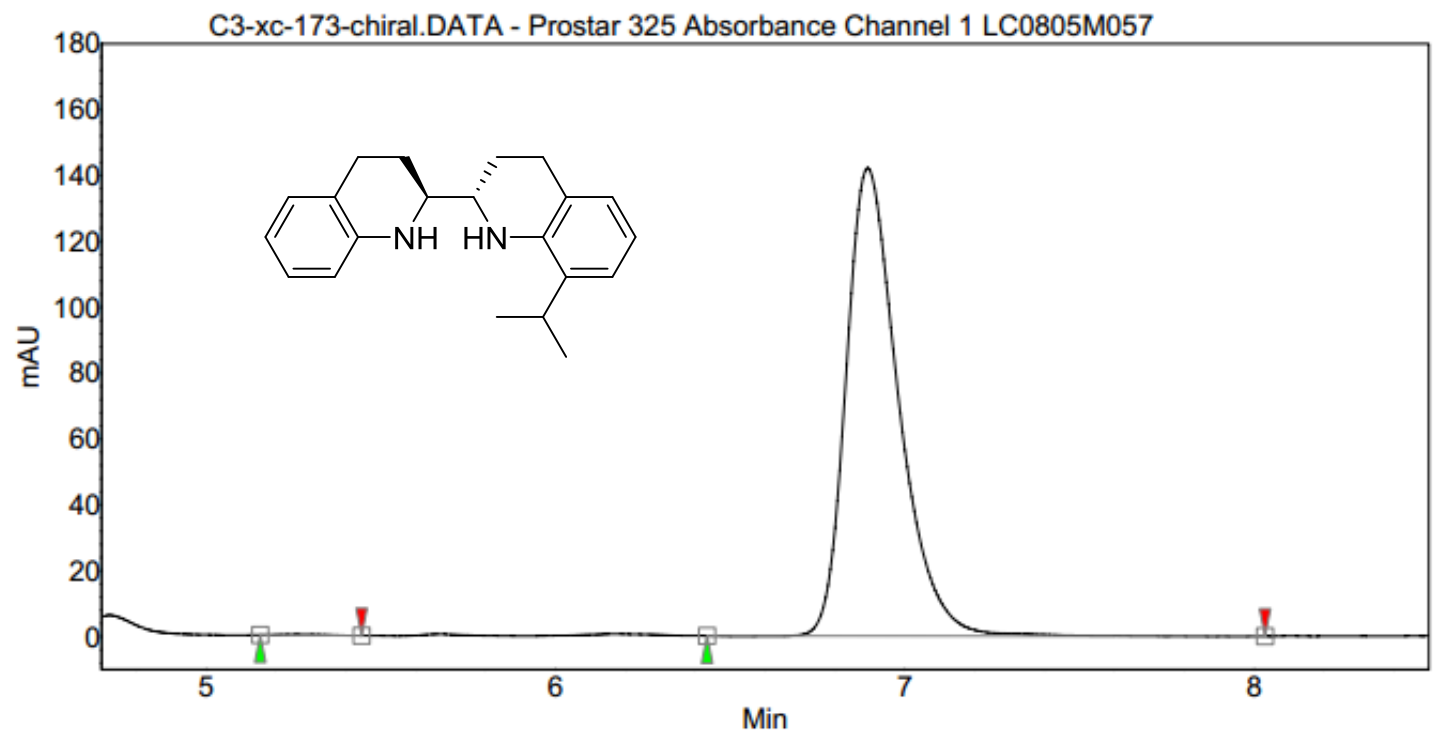

Peak results :

\begin{tabular}{|c|l|r|r|r|r|r|}
\hline Index & 文件名 & $\begin{array}{r}\text { 时间 } \\
\text { [Min] }\end{array}$ & $\begin{array}{r}\text { 数量 } \\
\text { [\% 面积 }\end{array}$ & $\begin{array}{r}\text { 高度 } \\
\text { [mAU] }\end{array}$ & $\begin{array}{r}\text { Area } \\
\text { [mAU.Min] }\end{array}$ & $\begin{array}{r}\text { Area \% } \\
\text { [\%] }\end{array}$ \\
\hline \hline 2 & 未知 & 5.27 & 0.15 & 0.3 & 0.0 & 0.153 \\
\hline 1 & 未知 & 6.89 & 99.85 & 142.1 & 24.7 & 99.847 \\
\hline & & & & & & \\
\hline Total & & & 100.00 & 142.4 & 24.8 & 100.000 \\
\hline
\end{tabular}


Figure S57. HPLC spectra of compounds $\mathbf{2 g}$
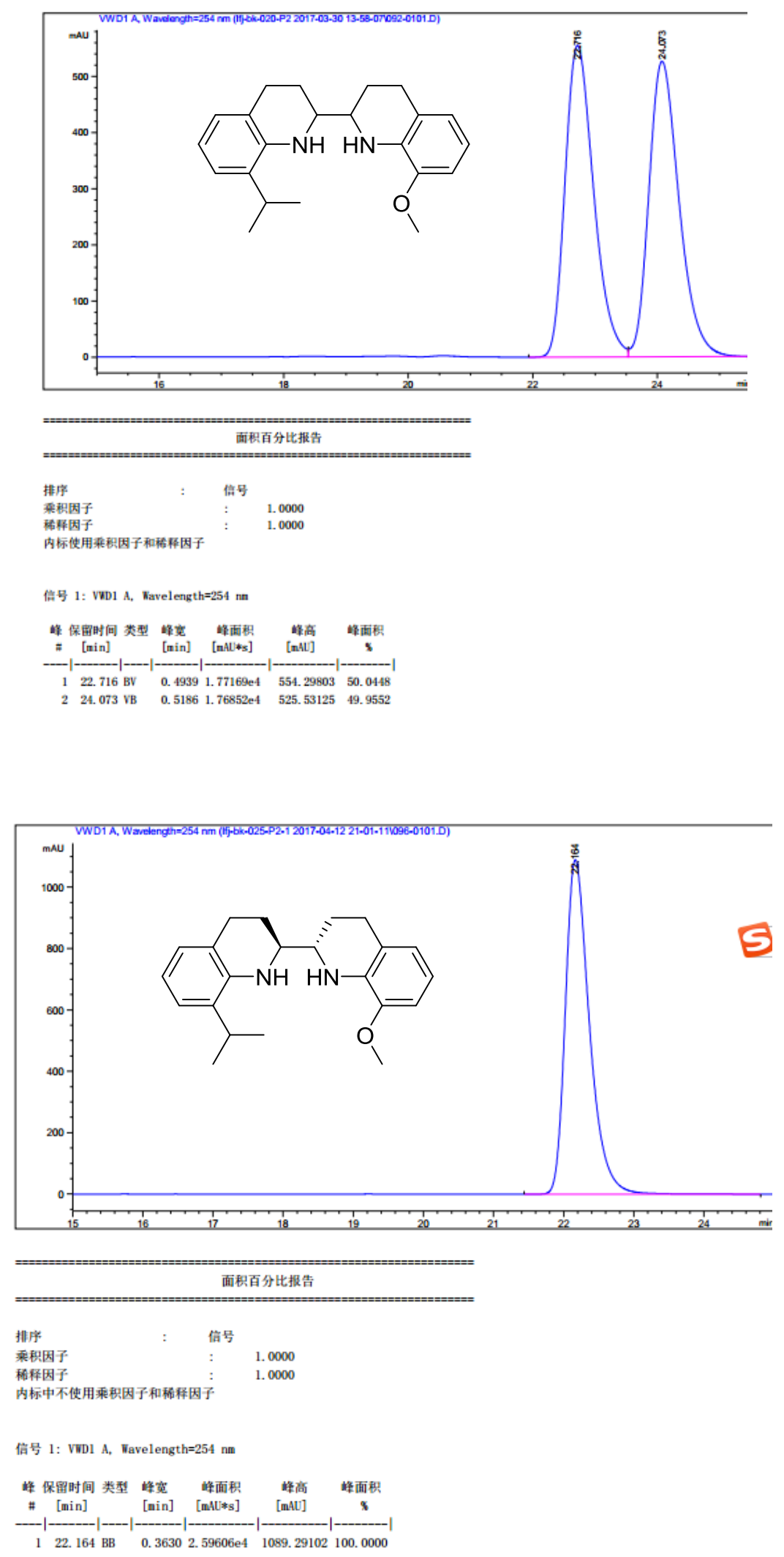
Figure S58. HPLC spectra of compounds $\mathbf{5 a}$

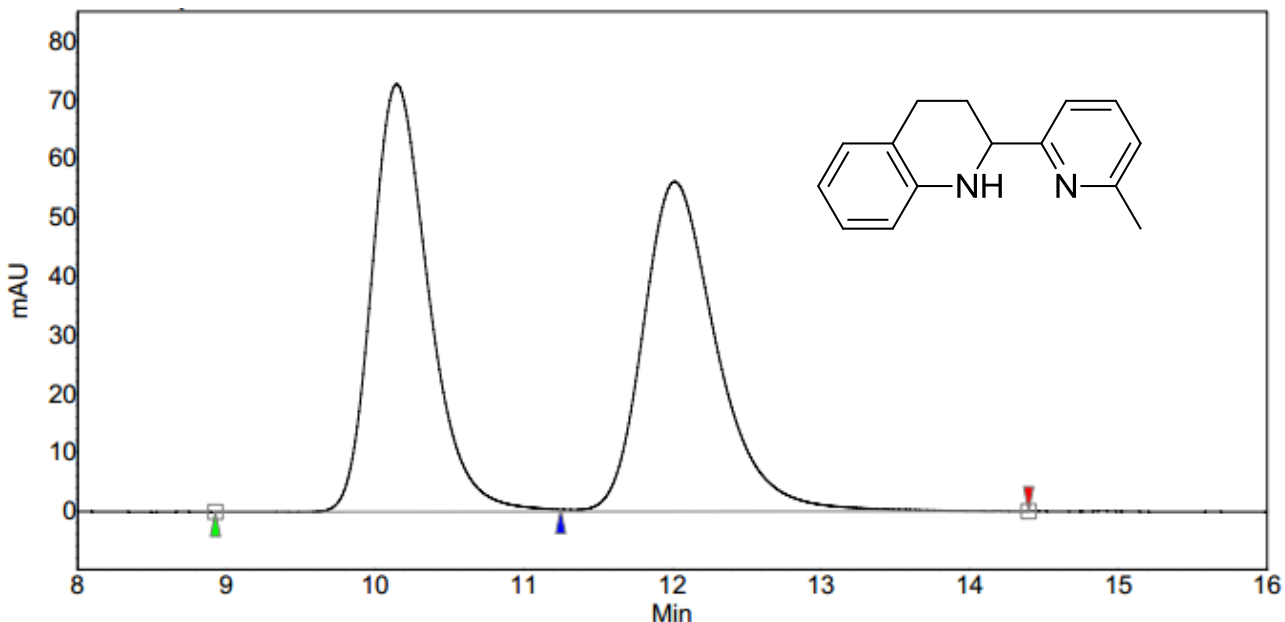

Peak results :

\begin{tabular}{|c|l|r|r|r|r|r|}
\hline Index & 文件名 & $\begin{array}{r}\text { 时间 } \\
\text { [Min] }\end{array}$ & $\begin{array}{r}\text { 数量 } \\
\text { [\% 面积] }\end{array}$ & $\begin{array}{r}\text { 高度 } \\
\text { [mAU] }\end{array}$ & $\begin{array}{r}\text { Area } \\
\text { [mAU.Min] }\end{array}$ & $\begin{array}{r}\text { Area \% } \\
{[\%]}\end{array}$ \\
\hline \hline 1 & 未知 & 10.15 & 49.87 & 72.8 & 32.1 & 49.872 \\
\hline 2 & 未先] & 12.01 & 50.13 & 56.1 & 32.3 & 50.128 \\
\hline & & & & & & \\
\hline Total & & & 100.00 & 128.9 & 64.5 & 100.000 \\
\hline
\end{tabular}

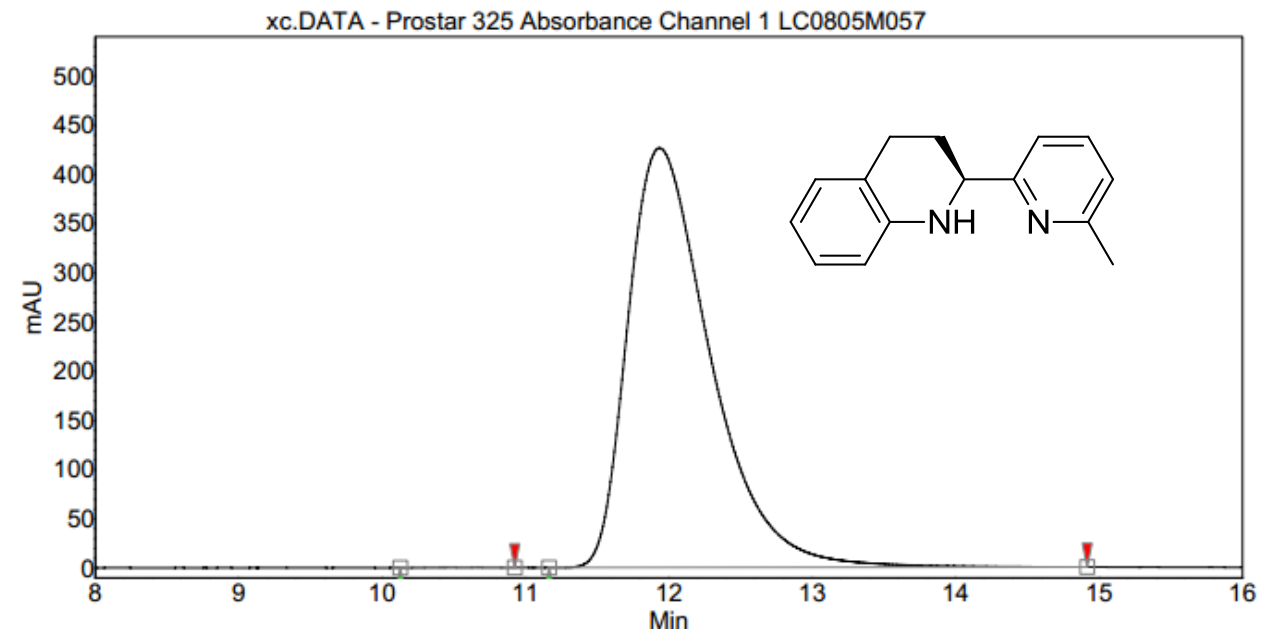

Peak results :

\begin{tabular}{|c|l|r|r|r|r|r|}
\hline Index & 文件名 & $\begin{array}{r}\text { 时间 } \\
{[\text { [Min] }}\end{array}$ & $\begin{array}{r}\text { 数量 } \\
\text { [\% 面积] }\end{array}$ & $\begin{array}{r}\text { 高度 } \\
\text { [mAU] }\end{array}$ & $\begin{array}{r}\text { Area } \\
\text { [mAU.Min] }\end{array}$ & $\begin{array}{r}\text { Area \% } \\
{[\%]}\end{array}$ \\
\hline \hline 1 & 未知 & 10.48 & 0.06 & 0.4 & 0.2 & 0.060 \\
\hline 2 & 未知 & 11.93 & 99.94 & 426.2 & 284.8 & 99.940 \\
\hline & & & & & & \\
\hline Total & & & 100.00 & 426.5 & 284.9 & 100.000 \\
\hline
\end{tabular}


Figure S59. HPLC spectra of compounds $\mathbf{5 b}$

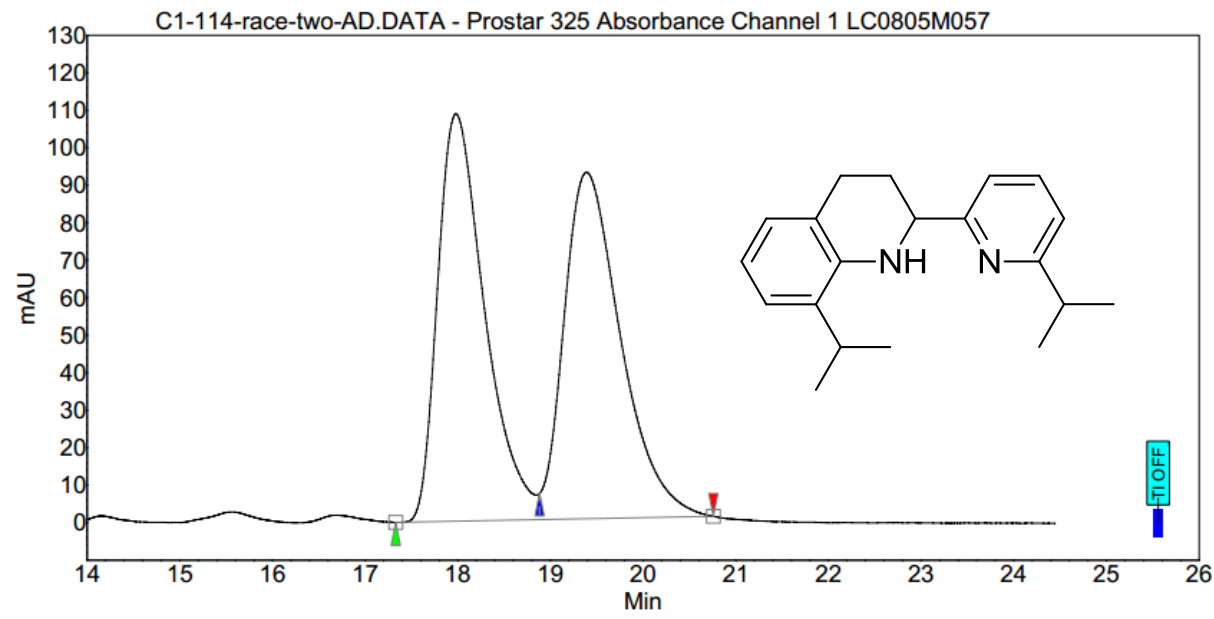

Peak results :

\begin{tabular}{|c|l|r|r|r|r|r|}
\hline Index & 文件名 & $\begin{array}{r}\text { 时间 } \\
\text { [Min] }\end{array}$ & $\begin{array}{r}\text { 数量 } \\
\text { [\% 面积] }\end{array}$ & $\begin{array}{r}\text { 高度 } \\
\text { [mAU] }\end{array}$ & $\begin{array}{r}\text { Area } \\
\text { [mAU.Min] }\end{array}$ & $\begin{array}{r}\text { Area \% } \\
\text { [\%] }\end{array}$ \\
\hline \hline 1 & 未知 & 17.98 & 49.47 & 108.6 & 64.1 & 49.468 \\
\hline 2 & 未知 & 19.39 & 50.53 & 92.4 & 65.4 & 50.532 \\
\hline & & & & & & \\
\hline Total & & & 100.00 & 201.1 & 129.5 & 100.000 \\
\hline
\end{tabular}

XC-IPR-liquid-0.6g.DATA - Prostar 325 Absorbance Channel 1 LC0805M057

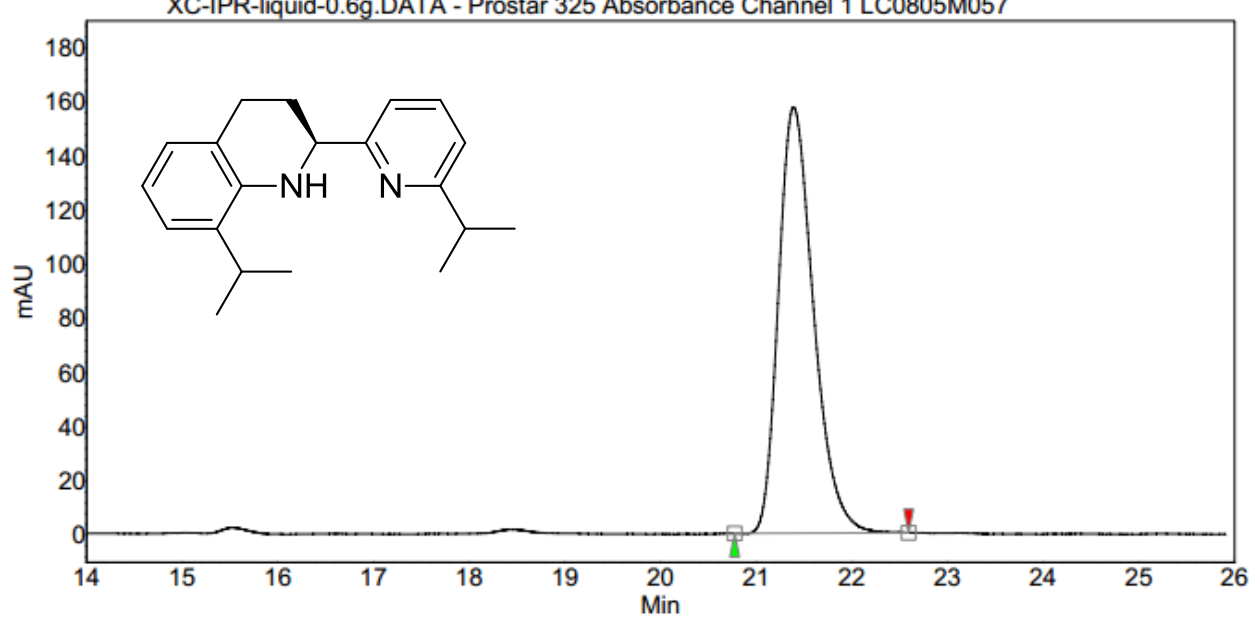

Peak results :

\begin{tabular}{|c|l|r|r|r|r|r|}
\hline Index & 文件名 & $\begin{array}{r}\text { 时间 } \\
\text { [Min] }\end{array}$ & $\begin{array}{r}\text { 数量 } \\
{[\% \text { 面积] }}\end{array}$ & $\begin{array}{r}\text { 高度 } \\
\text { [mAU] }\end{array}$ & $\begin{array}{r}\text { Area } \\
\text { [mAU.Min] }\end{array}$ & $\begin{array}{r}\text { Area \% } \\
{[\%]}\end{array}$ \\
\hline \hline 1 & 未知 & 21.39 & 100.00 & 157.4 & 67.0 & 100.000 \\
\hline & & & & & & \\
\hline Total & & & 100.00 & 157.4 & 67.0 & 100.000 \\
\hline
\end{tabular}


Figure S60. HPLC spectra of compounds $\mathbf{5 c}$

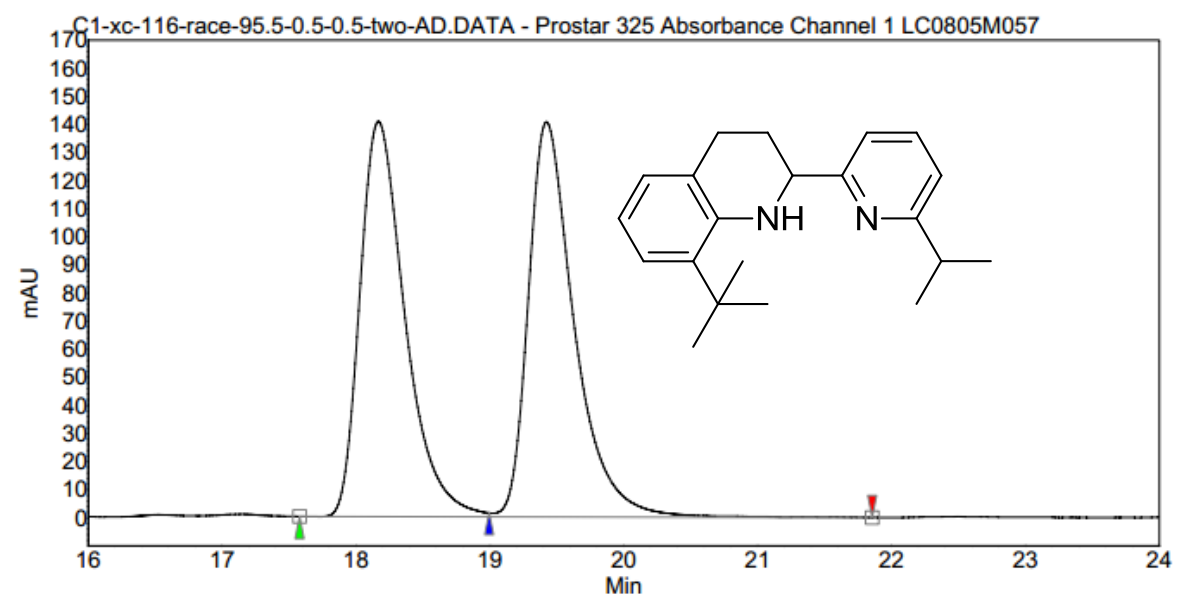

Peak results :

\begin{tabular}{|c|l|r|r|r|r|r|}
\hline Index & 文件名 & $\begin{array}{r}\text { 时间 } \\
\text { [Min] }\end{array}$ & $\begin{array}{r}\text { 数量 } \\
\text { [\% 面积] }\end{array}$ & $\begin{array}{r}\text { 高度 } \\
\text { [mAU] }\end{array}$ & $\begin{array}{r}\text { Area } \\
\text { [mAU.Min] }\end{array}$ & $\begin{array}{r}\text { Area \% } \\
{[\%]}\end{array}$ \\
\hline \hline 1 & 未知 & 18.17 & 49.45 & 140.6 & 54.9 & 49.448 \\
\hline 2 & 未和 & 19.42 & 50.55 & 140.6 & 56.1 & 50.552 \\
\hline & & & & & & \\
\hline Total & & & 100.00 & 281.2 & 111.0 & 100.000 \\
\hline
\end{tabular}

C1-xc-154-solid-1.DATA - Prostar 325 Absorbance Channel 1 LC0805M057

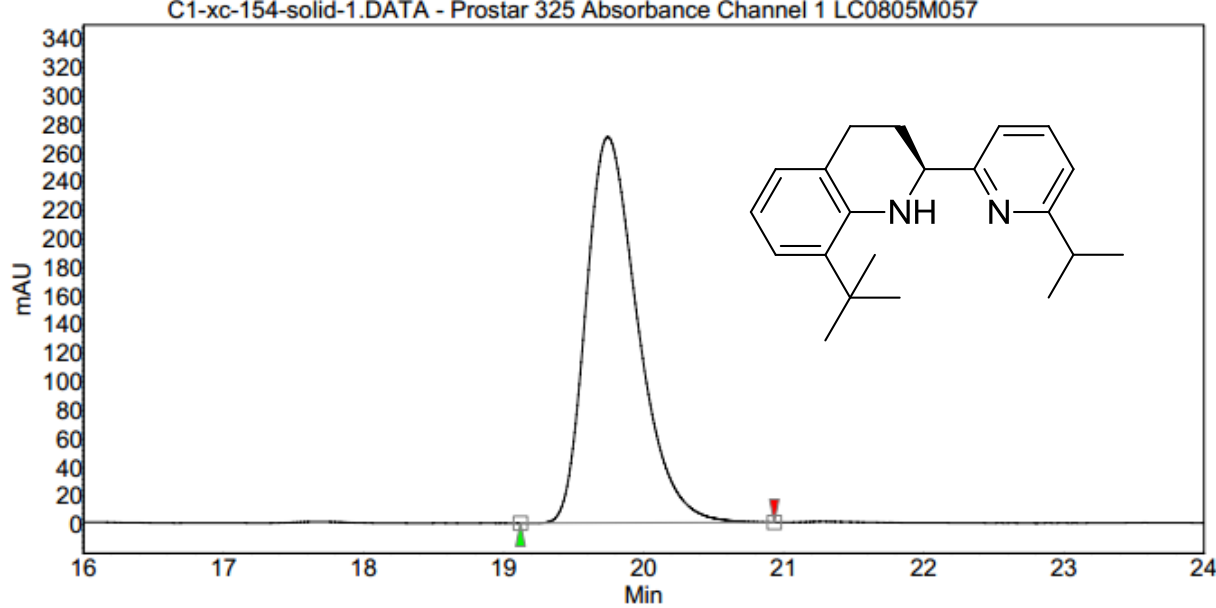

Peak results :

\begin{tabular}{|c|c|r|r|r|r|r|}
\hline Index & 文件名 & $\begin{array}{r}\text { 时间 } \\
\text { [Min] }\end{array}$ & $\begin{array}{r}\text { 数量 } \\
\text { [\% 面积] }\end{array}$ & $\begin{array}{r}\text { 高度 } \\
\text { [mAU] }\end{array}$ & $\begin{array}{r}\text { Area } \\
\text { [mAU.Min] }\end{array}$ & $\begin{array}{r}\text { Area \% } \\
{[\%]}\end{array}$ \\
\hline \hline 1 & 未知 & 19.74 & 100.00 & 270.3 & 115.9 & 100.000 \\
\hline & & & & & & \\
\hline Total & & & 100.00 & 270.3 & 115.9 & 100.000 \\
\hline
\end{tabular}


Figure S61. HPLC spectra of compounds $\mathbf{1 5}$

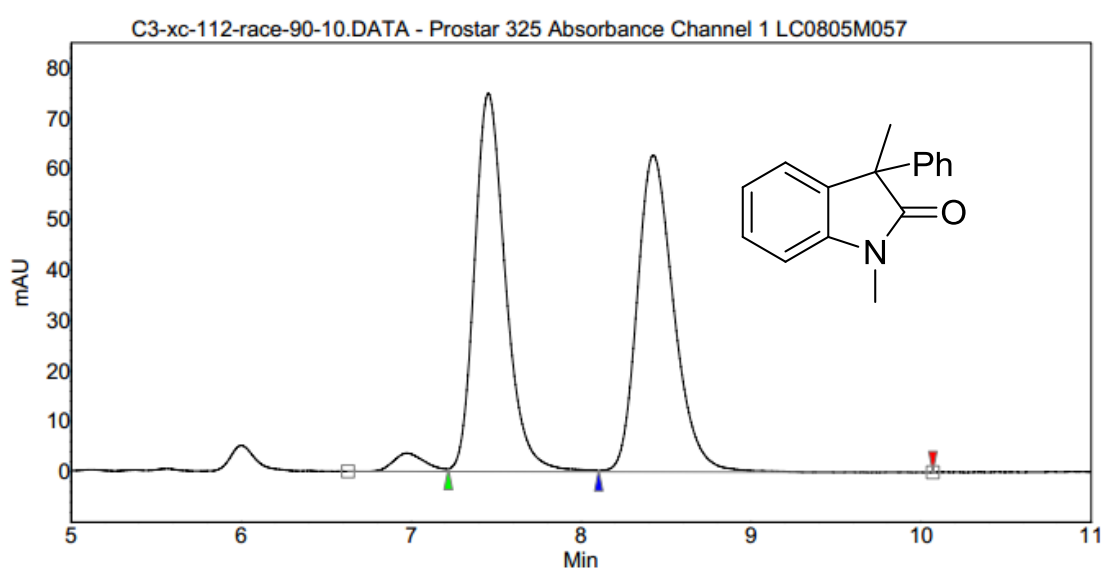

Peak results :

\begin{tabular}{|c|l|r|r|r|r|r|}
\hline Index & 文件名 & $\begin{array}{r}\text { 时间 } \\
\text { [Min] }\end{array}$ & $\begin{array}{r}\text { 数量 } \\
\text { [\% 面积 }\end{array}$ & $\begin{array}{r}\text { 高度 } \\
\text { [mAU] }\end{array}$ & $\begin{array}{r}\text { Area } \\
\text { [mAU.Min] }\end{array}$ & $\begin{array}{r}\text { Area \% } \\
{[\%]}\end{array}$ \\
\hline \hline 1 & 未知 & 7.45 & 50.30 & 75.0 & 15.8 & 50.303 \\
\hline 2 & 未知 & 8.43 & 49.70 & 62.8 & 15.6 & 49.697 \\
\hline & & & & & & \\
\hline Total & & & 100.00 & 137.8 & 31.5 & 100.000 \\
\hline
\end{tabular}

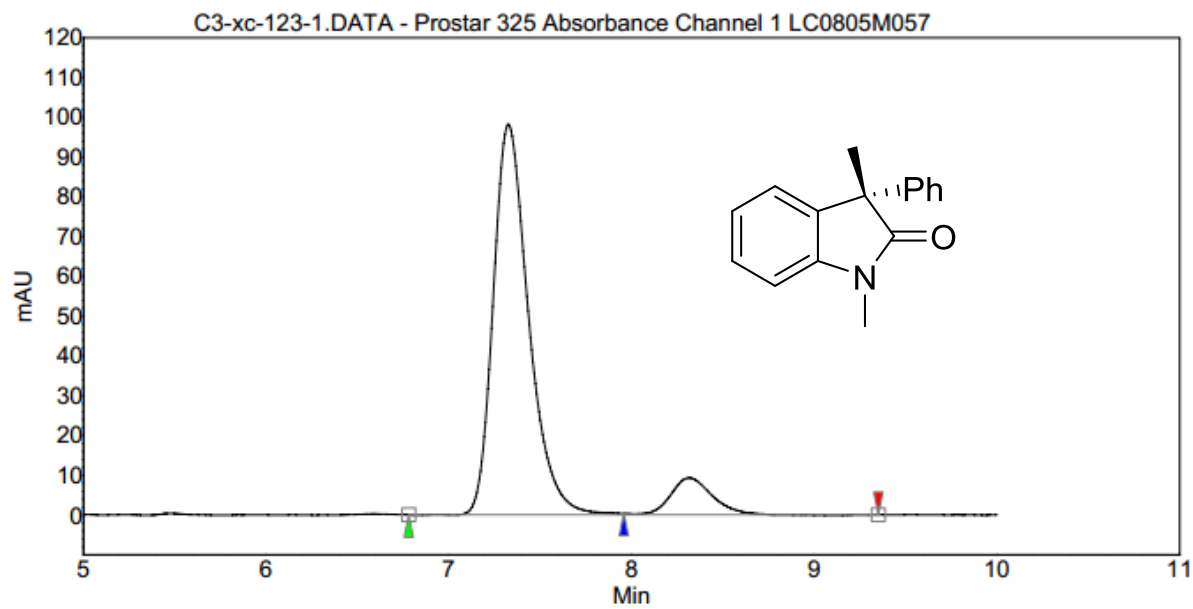

Peak results :

\begin{tabular}{|c|l|r|r|r|r|r|}
\hline Index & 文件名 & $\begin{array}{r}\text { 时间 } \\
\text { [Min] }\end{array}$ & $\begin{array}{r}\text { 数量 } \\
\text { [\% 面积] }\end{array}$ & $\begin{array}{r}\text { 高度 } \\
\text { [mAU] }\end{array}$ & $\begin{array}{r}\text { Area } \\
\text { [mAU.Min] }\end{array}$ & $\begin{array}{r}\text { Area \% } \\
{[\%]}\end{array}$ \\
\hline \hline 1 & 未知 & 7.33 & 90.21 & 98.1 & 22.0 & 90.214 \\
\hline 2 & 未知 & 8.31 & 9.79 & 9.2 & 2.4 & 9.786 \\
\hline & & & & & & \\
\hline Total & & & 100.00 & 107.4 & 24.4 & 100.000 \\
\hline
\end{tabular}




\section{Reference}

[1] a) Ohkuma, T.; Utsumi, N.; Tsutsumi, K.; Murata, K.; Sandoval, C.; Noyori, R. The Hydrogenation/Transfer Hydrogenation Network: Asymmetric Hydrogenation of Ketones with Chiral $\eta 6$-Arene/N-Tosylethylenediamine-Ruthenium(II) Catalysts. J. Am. Chem. Soc. 2006, 128, 8724-8725. b) Wang, T.; Zhuo, L.; Li, Z.; Chen, F.; Ding, Z.; He, Y.-M.; Fan, Q.-H.; Xiang, J.; Yu, Z.-X.; Chan, A. S. C. Highly Enantioselective Hydrogenation of Quinolines Using Phosphine-Free Chiral Cationic Ruthenium Catalysts: Scope, Mechanism, and Origin of Enantioselectivity. J. Am. Chem. Soc. 2011, 133, 9878-9891.

[2] a). Wang, H.; Pei, Y.; Bai, J.; Zhang, J. L.; Wu, Y. J.; Cui, X. L. Dimerization of Heteroaromatic $N$-oxides Under Metal-Free Conditions. $R S C A d v .2014,4$, 26244. b). Ma, W.; Zhang, J.; Xu, C.; Chen, F.; He, Y.-M.; Fan, Q.-H. Highly Enantioselective Direct Synthesis of Endocyclic Vicinal Diamines through Chiral Ru(diamine)-Catalyzed Hydrogenation of 2,2'-Bisquinoline Derivatives. Angew. Chem. Int. Ed. 2016, 55,12891-12894.

[3] Sylvester, K. T.; Wu, K.; Doyle, A. G. Mechanistic Investigation of the Nickel-Catalyzed Suzuki Reaction of N,O-Acetals: Evidence for Boronic Acid Assisted Oxidative Addition and an Iminium Activation Pathway. J. Am. Chem. Soc. 2012, 134, 16967-16970.

[4] Liu, Y.; Chen, F.; He, Y.-M.; Li, C.; Fan, Q.-H. Enantioselective Synthesis of Tunable Chiral Pyridine-Aminophosphine Ligands and Their Applications in Asymmetric Hydrogenation. Org. Biomol. Chem. 2019, 17, 5099-5105. 